\title{
Smart polymer brushes in nanopores: Towards controlled molecular transport through pore-spanning biomembranes
}


Smart polymer brushes in nanopores:

Towards controlled molecular transport through pore-spanning biomembranes 
The work described in this thesis has been performed at the Materials Science and Technology of Polymers (MTP) group, which is part of the Faculty of Science and Technology at the University of Twente, Enschede, The Netherlands. The research was financially supported by the MESA+ Institute for Nanotechnology of the University of Twente and by the European Commission through the FP7 project ASMENA (Grant No. CF-FP 214666-2).

Members of the committee:

\begin{tabular}{|c|c|c|}
\hline Chairman & Prof. dr. G. van der Steenhoven & $\begin{array}{l}\text { University of Twente, Enschede, } \\
\text { The Netherlands }\end{array}$ \\
\hline Promotor & Prof. dr. G.J. Vancso & $\begin{array}{l}\text { University of Twente, Enschede, } \\
\text { The Netherlands }\end{array}$ \\
\hline Assistant promotor & Dr. M.G. Santonicola & $\begin{array}{l}\text { Sapienza University of Rome, } \\
\text { Italy }\end{array}$ \\
\hline \multirow[t]{5}{*}{ Members } & Prof. dr. E. Reimhult & $\begin{array}{l}\text { University of Natural Resources } \\
\text { and Life Sciences, Vienna, Austria }\end{array}$ \\
\hline & Prof. dr. C. Vebert & $\begin{array}{l}\text { University of Geneva, } \\
\text { Switzerland }\end{array}$ \\
\hline & Prof. dr. D.C. Nijmeijer & $\begin{array}{l}\text { University of Twente, Enschede, } \\
\text { The Netherlands }\end{array}$ \\
\hline & Prof. dr. R.G.H. Lammertink & $\begin{array}{l}\text { University of Twente, Enschede, } \\
\text { The Netherlands }\end{array}$ \\
\hline & Dr. S. Le Gac & $\begin{array}{l}\text { University of Twente, Enschede, } \\
\text { The Netherlands }\end{array}$ \\
\hline
\end{tabular}

ISBN: 978-90-365-0772-1

DOI: $10.3990 / 1.9789036507721$

Copyright (C) Wilma de Groot, Enschede, The Netherlands 2013.

No part of this work may be reproduced by print, photocopy, or any other means without the permission in writing from the publisher.

Cover design by Geneviève Rietveld.

Printed by Ipskamp Drukkers in Enschede, The Netherlands. 


\title{
SMART POLYMER BRUSHES IN NANOPORES: TOWARDS CONTROLLED MOLECULAR TRANSPORT THROUGH PORE-SPANNING BIOMEMBRANES
}

\author{
PROEFSCHRIFT \\ ter verkrijging van \\ de graad van doctor aan de Universiteit Twente, \\ op gezag van de rector magnificus, \\ prof. dr. H. Brinksma, \\ volgens besluit van het College voor Promoties \\ in het openbaar te verdedigen \\ op donderdag 12 december 2013 om 12.45 uur \\ door \\ Gesina Wilhelmina de Groot \\ geboren op 2 januari 1983 \\ te Den Ham (Ov.)
}


Dit proefschrift is goedgekeurd door:

Promotor:

Prof. dr. G. Julius Vancso

Assistent-promotor:

Dr. M. Gabriella Santonicola 


\section{Contents}

\section{Chapter 1 Towards controlled molecular transport}

in bioassays with macromolecular nanotechnology 1

1.1 Introduction 1

1.2 Scope of this thesis 3

1.3 References 5

Chapter 2 Smart polymer brushes in

$\begin{array}{ll}\text { nanopores for controlled molecular gating } & 7\end{array}$

2.1 Polymer brushes 8

$\begin{array}{lll}2.2 & \text { Stimulus responsive polymer brushes } & 10\end{array}$

2.3 Polymer brush growth via surface-initiated atom transfer $\begin{array}{ll}\text { radical polymerization (SI-ATRP) } & 15\end{array}$

2.4 Characterization of (stimulus responsive) polymer brushes 18

2.5 (Stimulus responsive) polymer brushes in pores for controlled molecular transport or ion permeation $\quad 21$

2.6 Characterization of stimulus responsive polymer brushes in pores 27

2.7 References 29

Chapter 3 Pore-spanning lipid bilayers for membrane protein assays 33

3.1 Functional assays for membrane proteins 34

3.2 Supported and pore-spanning artificial lipid bilayers 35

3.2.1 Formation and characterization of artificial lipid bilayers 36

3.2.2 Supported lipid bilayers by stimulus responsive polymers 38

$\begin{array}{ll}3.2 .3 & \text { Free-standing lipid bilayers }\end{array}$

3.2.4 Polymer-supported, pore-spanning lipid bilayers 41

3.3 Integration of membrane proteins in artificial lipid bilayers 42

3.4 References $\quad 45$ 
Chapter 4 Reversible pH-controlled switching of

poly(methacrylic acid) grafts for functional biointerfaces

4.1 Introduction 50

4.2 Results and Discussion 52

4.2.1 PMAA brush growth and characterization from silicon surfaces 52

4.2.2 Characterization of $\mathrm{pH}$-sensitive behavior of PMAA brushes 55

4.3 Conclusions 62

4.4 Experimental Section 62

$\begin{array}{lll}4.5 & \text { References } & 65\end{array}$

Chapter 5 Switching transport through nanopores with

pH-responsive polymer brushes for controlled ion permeability $\quad 67$

5.1 Introduction 68

5.2 Results and Discussion 70

5.2.1 Functionalization and characterization of

nanoporous platforms with pH-responsive PMAA brushes $\quad 70$

5.2.2 Controlled nanopore gating function by

$\begin{array}{ll}\text { pH-responsive PMAA brushes } & 75\end{array}$

5.3 Conclusions 81

5.4 Experimental Section $\quad 82$

5.5 References $\quad 85$

Chapter 6 Smart polymer brush structures for guiding the self-assembly of pore-spanning lipid bilayers with integrated membrane proteins $\quad 89$

6.1 Introduction 90

6.2 Results and Discussion $\quad 92$

6.2.1 Functionalization of nanopore chips with NTA-modified PMAA brushes 92

6.2.2 Fluorescence confocal microscopy of nanopore-spanning lipid bilayers 96

6.2.3 Electrochemical impedance spectroscopy of single-nanopore spanned lipid membranes $\quad 99$

6.2.4 Nanopore-spanning lipid bilayers with integrated His-tagged membrane proteins $\quad 100$

$\begin{array}{lll}6.3 \text { Conclusions } & 102\end{array}$

6.4 Experimental Section 103

$\begin{array}{lll}6.5 & \text { References } & 107\end{array}$ 
7.1 Outlook

7.2 References

Summary

Samenvatting

Dankwoord

121

About the author

125 


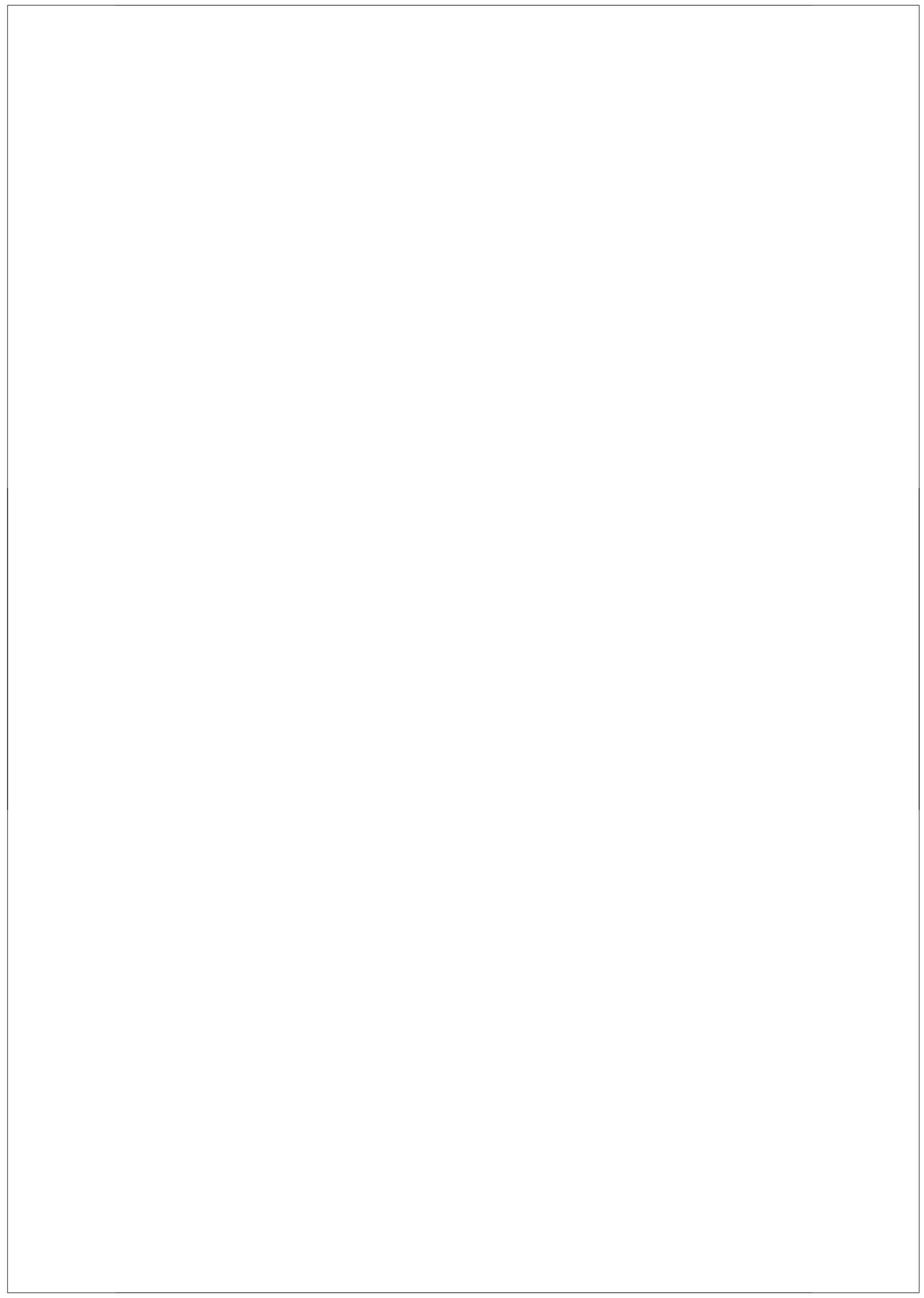




\section{Chapter 1}

\section{Towards controlled molecular transport in bioassays with macromolecular nanotechnology}

\subsection{Introduction}

The field of macromolecular nanotechnology offers a variety of opportunities to engineer devices with novel functions at the nanometer length scale. Especially for biotechnological devices, the integration of stimulus responsive polymers is an interesting strategy for controlling the properties of the integrated platforms, as these polymers can adapt their conformation and/or function by responding to changes in their surrounding environment. ${ }^{1}$

One method to obtain stable and robust polymer-modified devices is by the covalent binding of polymer chains to solid surfaces. Two strategies can be followed to immobilize polymer chains on a surface, namely 'grafting to' and 'grafting from'. In the 'grafting to' approach, polymer chains are synthesized in bulk and bound to the surface. The 'grafting from' approach starts, on the other hand, with the attachment of initiator molecules on the surface followed by a surface-initiated polymerization. ${ }^{2}$ The 'grafting from' approach results in so-called polymer brushes consisting of macromolecules that are covalently bound to a surface with a high enough grafting density. The high grafting density causes the polymer chains to stretch away from the surface to avoid overlap with their neighboring chains thereby forming a thick and dense polymer brush. When stimulus responsive polymers are used for the grafting, smart macromolecular architectures can be generated on surfaces whose macroscopic properties, e.g. wettability, adhesion and friction, can be controlled by varying an external stimulus, such as temperature, $\mathrm{pH}$ or electromagnetic field. After the discovery of stimulus responsive polymer films, most studies were focused on synthesis and characterization of the switchable properties, while nowadays there is a shift towards targeted applications of these smart polymer films. ${ }^{3,4}$

An interesting application of stimulus responsive polymers at the nanoscale consists of grafting polymer chains in confined environments, such as nanopores. Pores functionalized with smart polymer brushes can be used in micro- and nanofluidics as 
valves, filters and pumps. Flow control by stimulus responsive polymer grafts in polymeric membranes was first demonstrated by Ito et al. in the nineties. ${ }^{5}$ Recent developments in nanofabrication made it possible to generate solid-state nanopore membranes with a predefined geometry and pore array arrangement. Heyderman et al. produced thin silicon-based membranes with nanopores having diameters between 85 to $440 \mathrm{~nm},{ }^{6}$ whereas Reimhult et al. generated nanoporous films with pore diameters at the sub-100 nanometer scale. ${ }^{7}$ Functionalizing these solid-state nanopores with smart polymer brushes already showed the potential application of these platforms for controlled ion and molecular transport. ${ }^{8}$ This can be applied in new fields such as bioassays in lab-on-a-chip applications.

Devices based on nanopore arrays modified with smart polymer brushes can be especially useful in the development of functional assays for membrane proteins. Such assays would provide knowledge on membrane proteins as drug targets, ${ }^{9}$ and would shorten time and cost in drug development. ${ }^{10}$ In these platforms, several tasks need to be fulfilled by the nanopores functionalized with stimulus responsive polymers. The first task is to control the gating function of the nanopores to screen membrane proteins independent of each other, with respect to ions and low-molecular-weight drug candidates, by an external stimulus. This stimulus should result in a conformational and/or charge change of the polymer chains, so there is a reversible switching between 'on' and 'off' states of the nanopores. ${ }^{11} \mathrm{~A}$ second task that needs to be fulfilled by the device is the formation of artificial lipid bilayer membranes over the pores starting from liposomes rupture for the integration of membrane proteins. The spontaneous rupture of liposomes can be induced in several ways, e.g. by specific surface chemistry, vesicle size, temperature and/or osmotic pressure. ${ }^{12}$ Advanced polymer architectures can also facilitate the rupture and fusion of liposomes into functional, that is fluid, lipid bilayers, ${ }^{13}$ and support the free-standing lipid bilayer over the pores at the same time. By providing a stable support to the lipid bilayer assembly, polymer brushes can positively affect the long-term stability and therefore enhance the life-time of the device. As last task, specific bioconjugation methods can be applied to the side groups of the polymer brush $^{14}$ to provide anchor points for the membrane proteins integrated in the liposomes. The proteins can then be positioned specifically above the pores with both sides accessible for screening purposes. All these tasks are explored in this thesis. 


\subsection{Scope of this thesis}

The work described in this thesis is focused on the functionalization of nanopores with pH-responsive poly(methacrylic acid) (PMAA) brushes, specifically on nanoporous platforms to be used for drug screening. Controlled transport of ions and low-molecularweight molecules, pore-spanning lipid bilayers and positioning of membrane proteins over pores were all achieved by modification of the nanopores with PMAA brushes.

Chapter 2 provides the literature background covering synthesis and characterization of polymer brushes, as well as examples of stimulus responsive polymer brushes applied to control transport across pores. The chapter starts with the theory of polymer brushes followed by recent examples of surface functionalization with stimulus responsive polymer brushes. Subsequently, several synthetic routes to obtain polymer brushes via the 'grafting from' approach ${ }^{15}$ are discussed focusing specially on surface-initiated atom transfer radical polymerization (SI-ATRP). ${ }^{16}$ The characterization of polymer brushes using a variety of experimental methods, determination of the brush height and chain length, and characterization of their stimulus responsive behavior, receives ample treatment. Finally, the chapter ends with a description of the synthesis and characterization of polymer brushes in pores.

A literature overview focusing on pore-spanning lipid bilayers is provided in Chapter 3. This chapter includes also the framework in which the work presented in this thesis was performed. The development of smart polymer brushes in order to control molecular transport across nanopores is part of the European Union Seventh Framework Programme (FP7) 'Functional assays for membrane protein on nanostructured supports' (ASMENA), through which this PhD project was funded. First, membrane proteins and their role as drug targets are introduced, and the current limitations in the development of membrane protein assays are highlighted. Next, the formation and characterization of different artificial lipid bilayers are discussed, including polymer-supported systems. At the end of the chapter, a short overview of the integration of membrane proteins in artificial bilayers is presented.

The experimental part of this work presented in Chapter 4, describing the grafting of PMAA brushes from flat silicon surfaces using SI-ATRP. The growth kinetics of the polymer layers were investigated in water and water/methanol reaction mixtures. Adding methanol to the ATRP media was chosen to improve the surface wettability by the polymerization mixture, and so polymer grafting, in confined spaces in the view of subsequent experiments. The dissociation behavior of the PMAA chains was followed by 
Fourier transform infrared (FTIR) spectroscopy in titration experiments, and the $\mathrm{pH}$ sensitive swelling and collapse of the brush was characterized by in situ ellipsometry. The fast and reversible switching of the PMAA films offers possibilities to implement them in miniaturized devices.

The PMAA brush synthesis in a water/methanol mixture was applied to several nanoporous platforms with wells (dead-end pores) or channels (pores through). Chapter 5 describes the characterization of the PMAA brush grafting selectively inside the nanowells by atomic force microscopy (AFM) and scanning electron microscopy (SEM). AFM imaging was further used to observe the swelling of the PMAA brush inside the wells by varying the $\mathrm{pH}$ of the liquid environment from 4 to 8 . Platforms with nanochannels were functionalized with PMAA brushes and investigated by cyclic voltammetry at varied $\mathrm{pH}$ values in the Laboratory of Biosensors and Bioelectronics headed by Prof. János Vörös at ETH Zürich (Switzerland), one of the partners of the ASMENA project. The results of these measurements combined with diffusion experiments with a fluorescent dye demonstrated the controlled transport of ions and small molecules for gating platforms.

Chapter 6 presents the experimental work conducted with ASMENA collaborators Dr. Louis Tiefenauer and Sophie Demarche of the Laboratory of Biomolecular Research at Paul Scherrer Institut (Switzerland). Nanopore array chips functionalized with PMAA brush were used as supports for pore-spanning lipid bilayers with enhanced stability. The formation of the suspended artificial lipid bilayers was achieved by liposome rupture and fusion, and was confirmed by fluorescence recovery after photobleaching (FRAP) and electrochemical impedance spectroscopy (EIS). Besides supporting the lipid bilayer, the PMAA chains were functionalized with nitrilotriacetic acid (NTA) for immobilization of His-tagged membrane proteins. The localization of the membrane proteins near the pore edges and their integration in the pore-spanning lipid bilayer was imaged by fluorescence microscopy.

The outlook with a discussion of the current limitations to design screening devices for membrane proteins is given in Chapter 7. The future developments of the work presented in this thesis include addressing the switch on/off of individual pores for controlled transport across selected pores, analyzing the transport of ions and lowmolecular-weight drug candidates per single nanopore and testing the universal applicability of the designed assay platforms by integrating a variety of membrane proteins. 


\subsection{References}

1 Stuart, M. A. C.; Huck, W. T. S.; Genzer, J.; Muller, M.; Ober, C.; Stamm, M.; Sukhorukov, G. B.; Szleifer, I.; Tsukruk, V. V.; Urban, M.; Winnik, F.; Zauscher, S.; Luzinov, I.; Minko, S. Nat. Mater. 2010, 9, 101-113.

Barbey, R.; Lavanant, L.; Paripovic, D.; Schuwer, N.; Sugnaux, C.; Tugulu, S.; Klok, H. A. Chem. Rev. 2009, 109, 5437-5527. Luzinov, I.; Minko, S.; Tsukruk, V. V. Soft Matter 2008, 4, 714-725.

4 Azzaroni, O. J. Polym. Sci., Part A: Polym. Chem. 2012, 50, 3225-3258.

5 Ito, Y.; Park, Y. S. Polym. Adv. Technol. 2000, 11, 136-144.

6 Heyderman, L. J.; Ketterer, B.; Bachle, D.; Glaus, F.; Haas, B.; Schift, H.; Vogelsang, K.; Gobrecht, J.; Tiefenauer, L.; Dubochet, O.; Surbled, P.; Hessler, T. Microelectron. Eng. 2003, 67-8, 208-213.

7 Reimhult, E.; Kumar, K.; Knoll, W. Nanotechnology 2007, 18, 275303.

8 Tagliazucchi, M.; Szleifer, I. Soft Matter 2012, 8, 7292-7305.

9 Rask-Andersen, M.; Almén, M. S.; Schiöth, H. B. Nat. Rev. Drug Discovery 2011, 10, 579590.

10 Tiefenauer, L.; Demarche, S. Materials 2012, 5, 2205-2242.

11 Zhou, F.; Huck, W. T. S. Phys. Chem. Chem. Phys. 2006, 8, 3815-3823.

12 Reimhult, E.; Hook, F.; Kasemo, B. Langmuir 2003, 19, 1681-1691.

13 Tanaka, M.; Sackmann, E. Nature 2005, 437, 656-663.

14 Dai, J. H.; Bao, Z. Y.; Sun, L.; Hong, S. U.; Baker, G. L.; Bruening, M. L. Langmuir 2006, 22, 4274-4281.

15 Edmondson, S.; Osborne, V. L.; Huck, W. T. S. Chem. Soc. Rev. 2004, 33, 14-22.

16 Pyun, J.; Kowalewski, T.; Matyjaszewski, K. Macromol. Rapid Commun. 2003, 24, 10431059. 


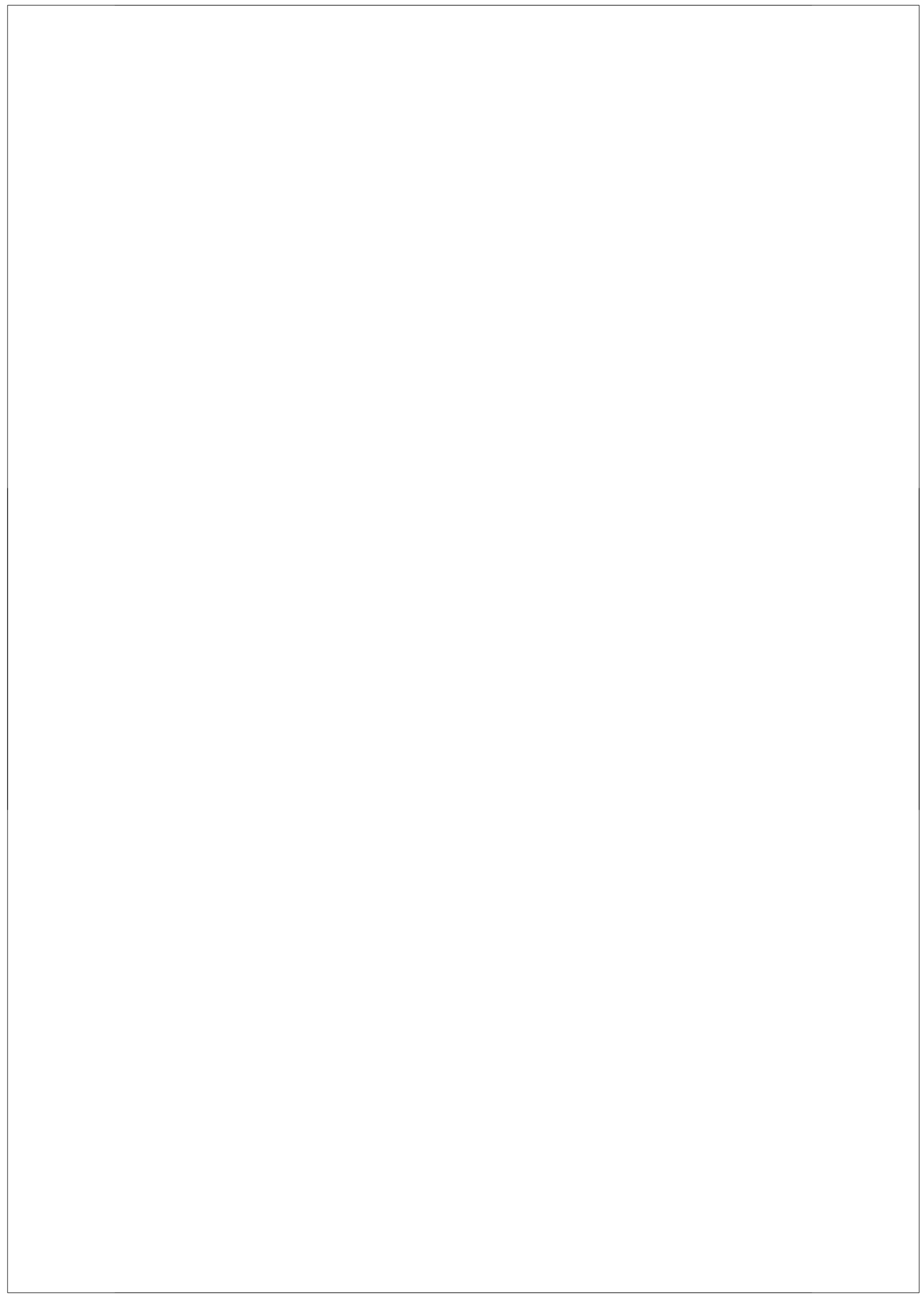




\section{Chapter 2}

\section{Smart polymer brushes in nanopores for controlled molecular gating}

This chapter provides the literature background of smart polymer brushes in nanopores, including their applications for controlled molecular transport through pores. In the first part of the chapter, the theory, synthesis and characterization of polymer brushes, including several surface-sensitive techniques, are discussed. Stimulus responsive polymer brushes and their application and characterization in channels are introduced next. The literature framework in this chapter is the foundation for the experimental work presented in Chapter 4 and Chapter 5. 


\subsection{Polymer brushes}

Polymer brushes belong to the class of surface tethered polymers, which can be anchored at flat or curved solid interfaces by physical or covalent chemical bonds and have a high enough grafting density and chain length to exhibit chain stretching. ${ }^{1}$ Representative examples for polymer attachment by physical forces include Van der Waals adsorption, electrostatic interactions and coupling via specific molecular forces (H-bonds). In this chapter we focus on covalent grafting of polymers. Other attachment approaches are well reviewed in the literature to which we refer. ${ }^{2,3}$

Covalent attachment can be achieved by two approaches including 'grafting to' and 'grafting from' coupling. In the 'grating to' method, reactive groups at the substrate surface and along (or at the end of) the polymer chain make covalent linkages. The 'grafting from' strategy encompasses first the chemical (or physical) deposition of polymerization initiator molecules, which usually form a molecular monolayer with reactive groups exposed for subsequent polymerization. Depending on the grafting density (chains per area) at the substrate and the chain length of the grafted polymer chains, two basic scenarios for the grafts can be realized, i.e. growth of a polymer mushroom or of a polymer brush. This is more explained in Figure 2.1a.

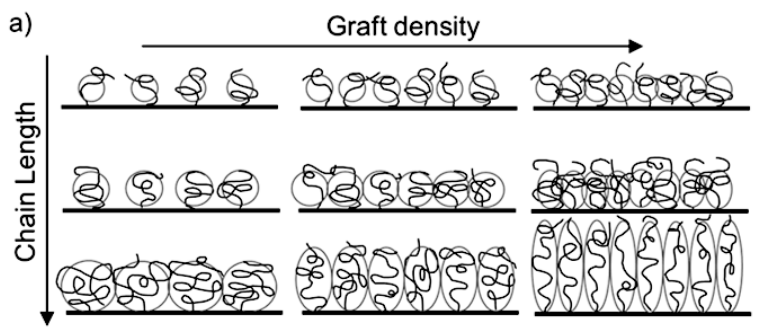

b)

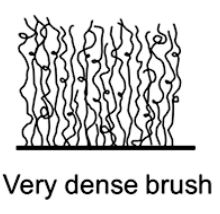

Figure 2.1: a) From grafted mushroom to polymer brush by increasing grafting density and chain length. b) Schematic of a very dense brush, only possible after 'grafting from' approach.

As can be seen in Figure 2.1a, along the increase of the grafting density and the polymer chain length polymer chains start to stretch away from the substrate to avoid overlapping and a mushroom configuration to a brush configuration takes place. Figure 2.1b gives a schematic example of a very dense polymer brush, which can only be obtained by the 'grafting from' approach where chains grow from small initiating molecules with a high density. Grafting with such a high density is not possible in the 'grafting to' approach where the large macromolecules are hindered by slow diffusion 
and reaching the substrate because of other macromolecules already linked to the substrate thus blocking neighboring sites where coupling would be needed to form a brush.

Mushroom to brush transition by varying grafting distance has been directly observed in an ingenious experiment by Genzer et al. in which they used an initiator gradient (the surface coverage of the initiator varied along the substrate) to grow polymers with a given chain length. As the distance between neighboring grafts decreased, a mushroomto-brush transition was observed. Figure 2.2 displays the figures from their experiments. ${ }^{4}$

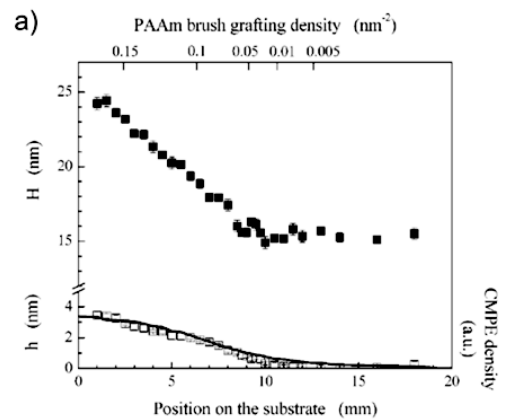

b)

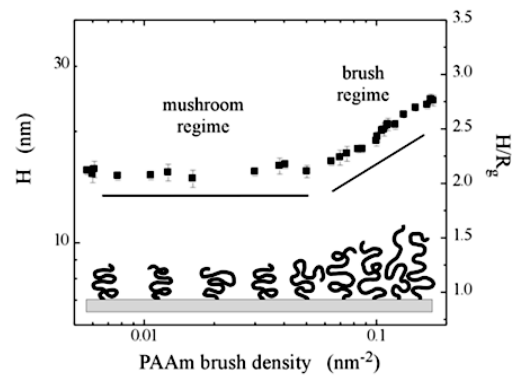

Figure 2.2: a) Dry ( $h$, open squares) and wet ( $H$, closed squares) polymer film thickness, and the initiator concentration (solid line) as a function of the position on the substrate. b) Wet polymer film thickness $(H)$ as a function of the grafting density, mushroom-to-brush transition. Adapted with permission from reference 4. Copyright 2002 American Chemical Society.

In Figure 2.2a Genzer et al. show that the concentration profile of initiator and the grafted polymer match each other and because they assume an almost same degree of polymerization for all the polymer chains, the gradient in thickness is caused by a difference in grafting density $(\sigma)$. Figure $2.2 \mathrm{~b}$ shows then the mushroom-to-brush transition. At low grafting density the polymer film thickness is independent of the grafting density $\left(H^{\sim} \sigma\right)$, the polymer grafts appear in a mushroom form. At high grafting density the polymer film thickness increases with increasing grafting density. By fitting the experimental data they observed a scaling between polymer film thickness and grafting density of $H^{\sim} \sigma^{1 / 3}$. This regime sets in for brush densities beyond the crossover.

Genzer et al. also published a review about surface grafted polymer chains with gradually varying physico-chemical properties displaying the applicability of these gradient grafts in a variety of fields. ${ }^{5}$ 
From the above it is clear that two important parameters that determine brush behavior encompass chain-chain grafting distance (or grafting density) and grafted chain length. These also determine the brush film thickness either in the dry state, or swollen by (good) solvents. In a subsequent section we describe some useful experimental approaches for the characterization of brush thickness, grafting density, and molar mass; here we briefly introduce some relevant theories that connect thickness and molecular parameters (chain length, grafting density).

The first molecular level theory that described the brush thickness in good solvents as a function of chain length was developed by Alexander and de Gennes. ${ }^{6,7}$ This model assumes a homogeneous distribution of segments within the polymer brush. De Gennes reasons that in good solvents the chains repel each other, and surface attachment terminates when the chains start to overlap (mushroom regime). The following expression for the brush thickness $L$ is obtained:

$$
\mathrm{L} / \mathrm{a} \sim \mathrm{N}(\mathrm{a} / \mathrm{b})^{2 / 3}
$$

where $a$ is an effective polymer segment diameter, and $b$ is the grafting distance (i.e. the grafting density $\sigma$ is $\sim 1 / b^{2}$ ). The density profile in this model exhibits a step-wise function: constant segment density vs. distance from the substrate until at the distance equals the brush thickness value the brush exposed interface is reached and the segment density drops to zero. In a subsequent study, Milner et al. extended the analysis of de Gennes-Alexander and refined the segment density distribution, obtaining a parabolic segment density profile. $^{8}$ Molecular dynamics simulations essentially confirmed the parabolic profile, and also showed that the free chain end is not excluded from regions near the substrate. ${ }^{9}$ Qualitative deviations from the Milner model only found for very high brush densities.

In good solvents the brushes swell, which results in chain expansion. The related loss of entropy is compensated by enthalpy gain due to osmotic effects. Force-brush compression curves strongly depend on grafting density thus they can be used for characterization purposes.

\subsection{Stimulus responsive polymer brushes}

Stimulus responsive polymer grafts have been used with great success to engineer the surfaces of materials. Stimulus responsive refers to materials that can adapt to variations in their surrounding environments. These external stimuli in the environment of the material can be, e.g. changes in $\mathrm{pH}$, temperature, mechanical force, or light. ${ }^{10}$ In 
the literature there are several examples of materials functionalized with stimulus responsive polymer brushes, some of them we will discuss in this section. These examples will display the broad field of applications of surface manipulation with stimulus responsive polymer thin films made by grafting. It is of importance that the switching of the surface properties are reversible, this reversibility should also be sustainable to several cycles of switching.

Temperature as stimulus is widely used, relatively easy to control and possible to apply in biomedical applications. Recently Roach et al. reviewed stimuli responsive materials for therapeutic applications and gave a schematic representation of a thermoresponsive graft as well as of its application to release cell sheets form surfaces. The schematic is presented in Figure 2.3. ${ }^{11}$

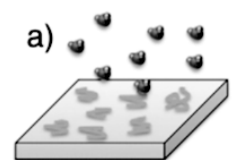

b)

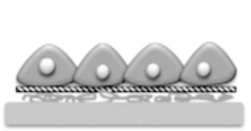

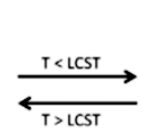
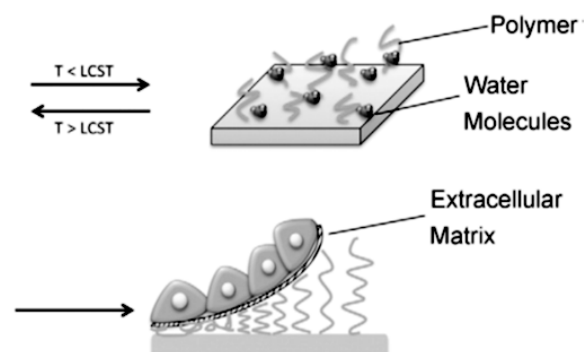

Figure 2.3: Schematic of a thermo-responsive graft. a) Reversible chains conformation change due to switching below and above about the LCST. b) Release of a cell sheet from a thermoresponsive graft. Reprinted with permission from reference 11. Copyright 2013 Elsevier.

The conformational change of the thermo-responsive chains in Figure 2.3a is caused by a change in solubility of the poly(N-isopropylacrylamide) (pNIPAM) chains in the solvent at different temperatures. In aqueous solutions uncharged polymer chains are stabilized by hydrogen bonds with the surrounding water molecules, but this effect decreases with increasing temperature. At the point where the system collapses, the lower critical solution temperature (LCST) is reached and the surface will change from hydrophilic to hydrophobic. This change is discussed in several publications about surfaces functionalized with pNIPAM grafts in combination with protein adsorption and cell attachment. As shown in Figure $2.3 \mathrm{~b}$ e.g. a cell sheet detaches from the thermoresponsive graft after lowering the temperature below the LCST. ${ }^{11,12}$

Our research group also grafted PNIPAM brushes and made use of a UV-initiated surface grafting. Here a patterned PNIPAM graft was obtained by putting a 
polydimethylsiloxane (PDMS) stamp with channels on a gold substrate during formation of an initiator monolayer. Therefore the initiator molecules are only covalently attached to the gold at the exposed areas. This patterned platform was used to investigate the swelling response of PNIPAM brushes by changing the temperature from above to below the LCST ( $32{ }^{\circ} \mathrm{C}$ for bulk pNIPAM). The response was imaged by in situ atomic force microscopy (AFM) measurements, which is displayed in Figure $2.4 .^{13}$

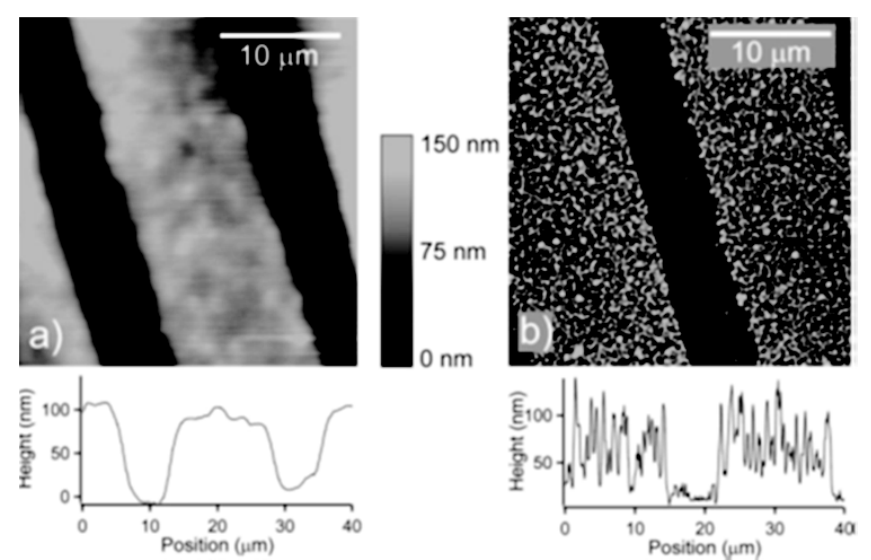

Figure 2.4: Height images from tapping-mode AFM and corresponding cross sections of patterned pNIPAM grafts. Captured at a) $31{ }^{\circ} \mathrm{C}$ and b) $36^{\circ} \mathrm{C}$. Reprinted with permission from reference 13. Copyright 2007 John Wiley and Sons.

This experiment clearly shows the collapse of the PNIPAM chains above the LCST into individual aggregated globular features, which displays the potential for these thermoresponsive platforms for biological applications. ${ }^{13}$

Huck et al. used micro-patterned pNIPAM brushes earlier for applications in shortterm bioadhesion assays. They also made use of PDMS stamps with channels to microcontact print their initiator molecules. The thermo-responsive polymer brushes were grown by surface-initiated atom transfer radical polymerization (SI-ATRP), which is a well-known technique to graft polymer brushes from surfaces and will be discussed in section 2.3. The polymer brush transition from hydrophobic to hydrophilic was investigated by attachment of a bacterium, which is known for higher adhesion at hydrophilic surfaces. The micro-patterned pNIPAM brush surfaces were immersed in solutions of this bacterium at temperatures above and below the LCST of PNIPAM and fluorescence images were taken, which are shown in Figure 2.5. ${ }^{14}$ 


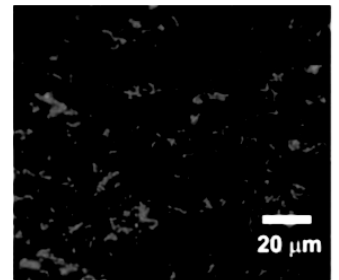

a)

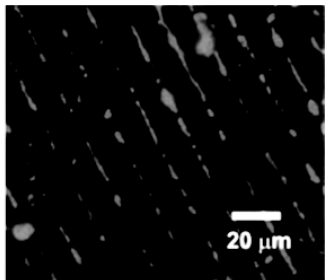

b)

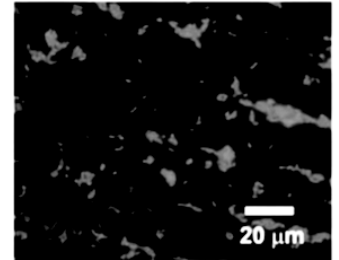

c)

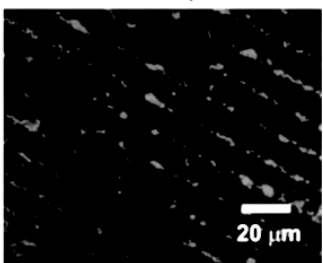

d)

Figure 2.5: Fluorescence images (light grey represents red) of patterned brush surfaces immersed for $1 \mathrm{~h}$ in bacterium solutions above $\operatorname{LCST}(\mathrm{a}, \mathrm{c})$ and below LCST $(\mathrm{b}, \mathrm{d})$. Reprinted with permission from reference 14. Copyright 2005 The Royal Society of Chemistry.

The images in Figure 2.5 display a non-specific binding above the LCST, which corresponds to a hydrophobic surface and therefore to a lower adhesion of the bacterium. After switching of the temperature below the LCST there is an accumulation of the bacterium at the pattern of pNIPAM brush. This approach of a thermo-responsive surface with multiple environments can lead to a variety of arrays and patterns that reversible adsorb or reject specific cell types. ${ }^{14}$

Besides thermo-responsive polymer brushes, there are many other stimulus responsive polymer brushes of which also some examples will be mentioned. The first example is grafting of a mixed polymer brush of hydrophobic and hydrophilic polymers to a polymer film with needles of micrometer size for control of surface wettability. Minko et al. reported this fabrication of a rough platform with reversible responsive surface properties. In Figure 2.6 a scheme of the surface morphology and the response of the surface to the solvents water and 1,4-dioxane is displayed. ${ }^{15}$ 


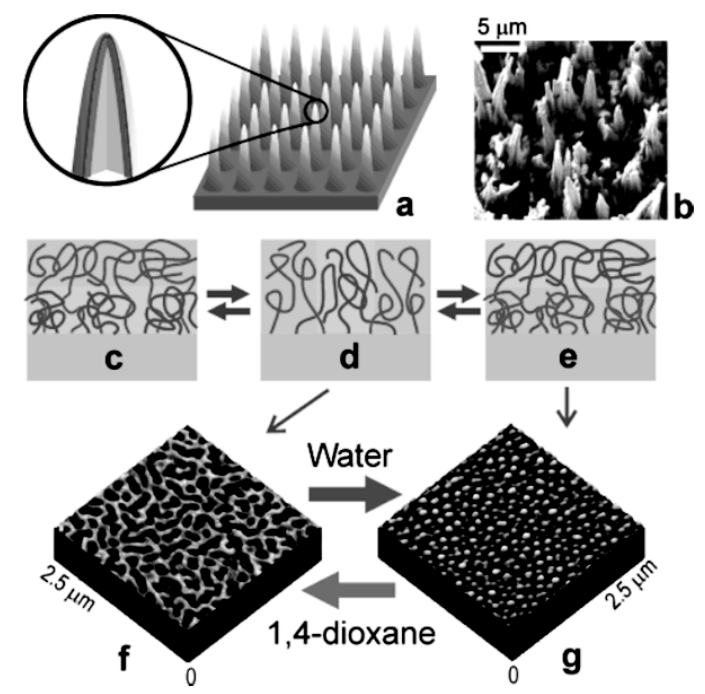

Figure 2.6: (a) Schematic of polymer film with needlelike surface morphology. (b) SEM image of etched polymer film. (c-e) Mixed polymer brush grafted to needles. Blue represents the hydrophilic polymers and red the hydrophobic polymers. (c, e) Response in selective solvents and (d) in a non-selective solvent. ( $f, g$ ) AFM images of model flat surfaces with mixed polymer brush exposed to 1,4-dioxane and water. Reprinted with permission from reference 15 . Copyright 2003 American Chemical Society.

The approach of Minko et al. shows that structuring a surface at two levels, including a micrometer size needle structure and a mixed polymer brush in the nanometer scale, is a smart way to tune surface wettability, and therefore adhesion, over a wide range from ultrahydrophobic to hydrophilic. ${ }^{15}$

The last example mentioned in this section, is work from Huck and co-workers. We note that there are other similar studies, especially in rapidly growing life science applications. ${ }^{16}$ Huck et al. used polyelectrolyte brushes to functionalize microcantilevers, which can be used in developing extremely sensitive chemical sensors and biosensors in microfluidic devices without changing the chemical environment. ${ }^{17}$ 


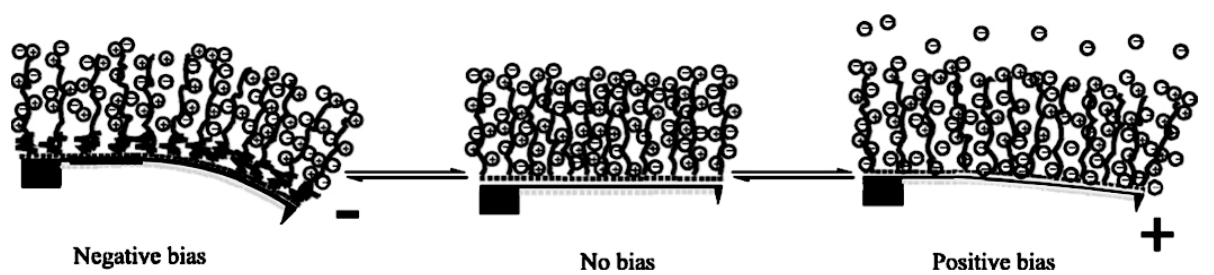

Figure 2.7: Microcantilever coated with a cationic polyelectrolyte brush on gold. A bias results in a deflection of the cantilever, where a negative bias gives a stronger deflection than a positive bias. Reprinted with permission from reference 17. Copyright 2008 American Chemical Society.

Applying a bias to the cantilever resulted in bending of the cantilever due to changes in the polymer brush close to the electrified surface. These conformational changes of the polymer chains are caused by the reorganization of ions. Huck et al. also developed a theoretical model to describe these observations, which matched with the experimental data. These polyelectrolyte brushes can be used in the electroactuation of cantilevers. ${ }^{17}$

Besides this example of Huck et al., there are also several other examples of applications where the surrounding of polyelectrolyte brushes is changed. Later this will be discussed in more detail as the work throughout this thesis is based on $\mathrm{pH}$-responsive poly(methacrylic acid) (PMAA) brushes, which is a weak polyelectrolyte polymer brush. ${ }^{18}$

2.3 Polymer brush growth via surface-initiated atom transfer radical polymerization (SI-ATRP)

Over the last decades significant progress has been achieved in surface-initiated polymerization approaches (see e.g. reference 3). Here we first provide a short overview of frequently used synthetic approaches, and then go in more detail concerning the SIATRP mechanism used to obtain brushes by the 'grafting from' approach. Different examples of surface-initiated polymerization techniques are displayed in Figure 2.8. 
a)

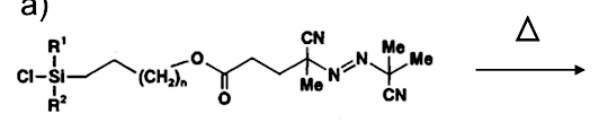

polymer brush growth from azo initiators

b)<smiles>[R]C1CC(CCC(C)C)CC1C(=C)CC(C)C</smiles>

ring-opening metathesis polymerization

d) (ROMP)

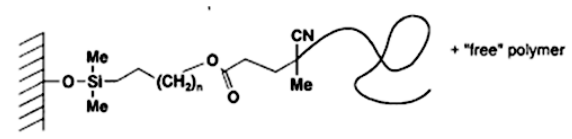

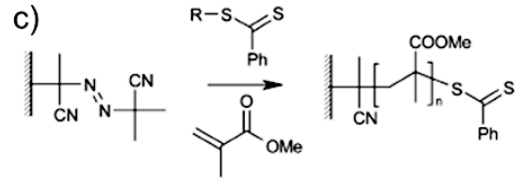

reversible addition fragmentation chain transfer (RAFT)<smiles>CC(C)CC(ON1C(C)(C)CCCC1(C)C)c1ccccc1</smiles>

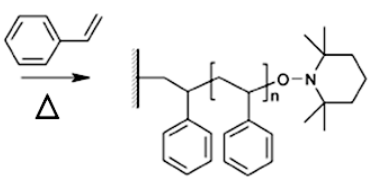

nitroxide-mediated radical polymerization (NMP)

Figure 2.8: Surface-initiated polymerization techniques. Traditional free radical approaches: a) grafting from azo-based initiators, and b) ring-opening metathesis polymerization (ROMP). Controlled 'living' free radical approaches: c) reversible addition fragmentation chain transfer (RAFT), and d) nitroxide-mediated radical polymerization (NMP). ${ }^{19-23}$ Reprinted with permission from reference 22. Copyright 1998 American Chemical Society. Adapted with permission from reference 23. Copyright 2004 The Royal Society of Chemistry.

In Figure 2.8a conventional azo initiators are presented, which could be attached to $\mathrm{OH}$ terminated surfaces, e.g. by the silane coupling as shown in Figure 2.8a. The azo initiator can be activated e.g by heat to obtain polymer chains covalently grafted to the substrate. $^{22}$ The chain length and its distribution can be estimated using mechanism kinetics models of addition polymerization considering confinement effects. This approach obviously does not allow one to prepare brushes with well-controlled molecular architecture i.e. chain length and block structures.

For these molecular architectures a better control of the polymer growth is required. In Figure 2.8b-d more controlled surface-initiated polymerization techniques are presented. First, ring-opening metathesis polymerization (ROMP) is shown in Figure 2.8b. In this example crystalline Si surfaces are first chlorinated and subsequently an alkene linker is coupled via a Grignard reaction. A ruthenium ROMP catalyst was then crossed onto this linker and the surfaces were immersed in a monomer solution. The 
thickness of the resulting polymer films grafted from the surfaces could be controlled by the concentration of the monomer in solution. ${ }^{21}$

Another polymerization technique, which can be applied for the synthesis of polymer brushes, is reversible addition fragmentation chain transfer (RAFT). This technique is a free radical controlled polymerization, which results in polymer brushes with controlled length and specific chain architectures. In the work presented in Figure 2.8c silicate surfaces were functionalized with a surface-immobilized azo initator. Methyl methacrylate brushes were subsequently grafted from these surfaces under RAFT conditions. Also the 'living' character of this polymerization technique was demonstrated, because bringing the substrates in contact with different monomer solutions resulted in block copolymer brushes. ${ }^{19}$

The last technique to mention, before going in more detail to SI-ATRP, is nitroxidemediated radical polymerization (NMP), which is also a controlled 'living' radical polymerization. This technique provides control over the molar mass, and also yields relatively low polydispersity of the grafted chains. In Figure $2.8 \mathrm{~d}$ an example of SI-NMP is displayed, the brush is grafted from surface-tethered alkoxyamines as the initiator molecules, which are e.g. more stable than azo-based initiators. First attempts to graft brushes from these initiating sites were unsuccessful, however by adding a small amount of 'free' initiator Husseman et al. were able to control the chain growth. Polystyrene brushes were the result and the polymer chains in solution could be easily removed by rinsing with the appropriate solvent. A disadvantage of SI-NMP encountered was the necessary high polymerization temperature. ${ }^{20}$

Just before Husseman et al. published their work, the first preliminary reports appeared about grafting of polymer brushes with atom transfer radical polymerization (ATRP). Nowadays, surface-initiated atom transfer radical polymerization (SI-ATRP) is the most frequently used technique for 'grafting from' polymer brushes. ${ }^{24}$ SI-ATRP provides an environment in which polymer brush growth is reproducible and yields robust polymer brush structures, that are well-defined in chain length and architecture. The grafting density can, in principle, be controlled by tuning the coverage of initiators attached to the substrates. The foundation is off course ATRP and the general scheme is presented in Figure 2.9. ${ }^{25-27}$ 


$$
R-X+M_{t}^{n}-Y / \text { Ligand } \frac{k_{\text {act }}}{k_{\text {deact }}} \underset{\text { monomer }}{\left(k_{p}\right)} \overbrace{}^{R} \dddot{k}_{t}{ }_{\text {termination }}+X-M_{t}^{n+1}-Y / \text { Ligand }
$$

Figure 2.9: Transition-Metal-Catalyzed ATRP. Reprinted with permission from reference 25. Copyright 2001 American Chemical Society.

ATRP was developed in 1995 by Professor K. Matyjaszewski and belongs to the group of controlled radical polymerizations (CRP). All CRP methods are based on dormant chains, in ATRP these are alkyl halides, and are initiated by the generation of free radicals, in ATRP this goes via a catalyzed reaction. As can be seen in Figure 2.9, the reversible redox process in ATRP is catalyzed by a transition metal complex $\left(\mathrm{M}_{\mathrm{t}}{ }^{n}\right.$ $\mathrm{Y} /$ Ligand, where $\mathrm{Y}$ may be another ligand or the counterion). In between the dormant chain $(\mathrm{R}-\mathrm{X})$ and the transition metal complex the transfer of a halide $(\mathrm{X})$ occurs, which results in a radical $(R \cdot)$ for propagation by addition of monomer and the transition metal complex in a different oxidation state $\left(X-M_{t}{ }^{n+1}-Y /\right.$ Ligand $) .{ }^{25,28}$

The initiating step depends on a rate constant of activation $\left(k_{\text {act }}\right)$, which is fast in ATRP, and together with a rapid reversible deactivation $\left(k_{\text {deact }}\right)$, these contribute to a uniform growth of all the polymer chains. Also radical-radical termination reactions are prevented by these two features, because there is a low amount of active radical chains and a high amount of dormant chains in the reaction mixture. The propagation reaction (rate constant $k_{p}$ ) happens in a similar way as in conventional radical polymerizations. Normally, in a well-controlled ATRP, a low percentage of the chains terminates (rate constant $\left.k_{t}\right)^{25}$

ATRP is also suitable for surface-initiated polymerizations from halide-containing initiators, as there is a low concentration of active radicals in the confined environment close to the substrate, which minimizes the interaction of chain ends. Therefore there are many examples in literature where SI-ATRP is used for polymer brush growth. This displays the versatility of the synthesis of (stimulus responsive) polymer brushes by SIATRP, which results in a wide variety of functionalized surfaces of materials. ${ }^{23,29}$

\subsection{Characterization of (stimulus responsive) polymer brushes}

The two most important molecular parameters that characterize a polymer brush include the length of the grafted chains (and its distribution), and the average distance between the anchoring points on the substrate (or the grafting density, i.e. chains $/ \mathrm{nm}^{2}$ ). These parameters define the thickness of brush layers when swollen by good solvents. 
As mentioned earlier, the first molecular level theory that described the brush thickness in good solvents as a function of chain length was developed by Alexander and de Gennes. ${ }^{6,7}$ Another brush model with a non-uniform segment density profile was developed by Milner, who predicted a parabolic concentration profile. We note, that it is very difficult to predict the segment density profile in brushes, many parameters that contribute to determining the ultimate thickness are often left beyond consideration of the models. ${ }^{8,30}$

There are several ways to determine the brush thickness, characterization tools as ellipsometry and AFM are widely used. ${ }^{24}$ Especially, AFM is used as an analytical tool since more complicated architectures of polymer brushes are being developed. ${ }^{27,31}$ Other techniques include scattering techniques, e.g. X-ray reflectivity (XRR) and neutron reflectivity. ${ }^{32}$ Péter et al. used XRR in order to follow the layer thickness changes of redox-responsive surface-grafted poly(ferrocenyldimethylsilane) monolayers upon electrochemical oxidation. ${ }^{33}$

Ellipsometry is a suitable and precise tool to determine the thickness of polymer brushes. Brush thicknesses can be defined in a dry environment, but with the use of a liquid cell, the swollen thickness of a stimulus responsive polymer brush in varied solvents can also be obtained. Kooij et al. used in situ ellipsometry to probe the changes of thermo-responsive polymer brushes by changing the surrounding environment of the polymer brushes. ${ }^{34}$

A convenient method to measure the polymer brush height by AFM is scratching of a polymer brush functionalized flat surface. Another method is by making use of patterned brushes to obtain a step height, however the pattern also influences the step height. A recent review by Chen et al. discusses the fabrication of patterned polymer brushes. ${ }^{35}$ Benetti et al. used the method of scratching and the result is displayed in Figure $2.10 .^{36}$ 


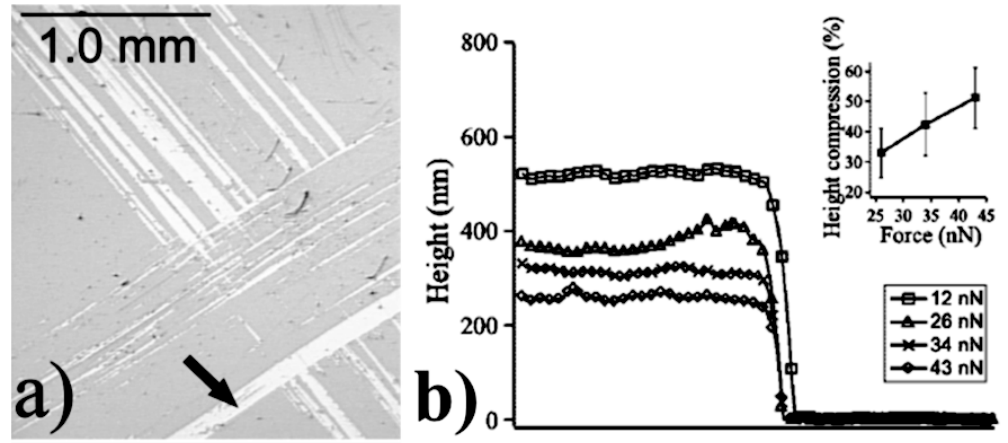

Figure 2.10: a) Scratched film of polymer brush. b) Cross section presenting the step height of the polymer brush at varied applied forces. Adapted with permission from reference 36. Copyright 2009 American Chemical Society.

As can be seen in Figure 2.10b, Benetti et al. took also into account the compressibility of the polymer brush by the force applied by the AFM tip. This was for investigation of polymer brushes obtained under exactly the same polymerization conditions, but starting with a different initiator monolayer to obtain a variety in grafting density, and therefore a different compressibility. This experiment showed that brush compression by the AFM tip plays also a role in the determination of the film thickness and can lead to an underestimation of the polymer brush height. ${ }^{36,37}$

The other important parameter of a polymer brush is the grafting density, which can be calculated from the dry thickness of the polymer brush and the molar mass of the polymer chains. After molar mass determination the grafting density can be calculated by:

$$
\sigma=\left(h \rho N_{a}\right) / M_{n}
$$

where $h$ is the dry brush thickness, $\rho$ is the bulk density of the brush composition , $N_{a}$ is Avogadro's number and $M_{n}$ is the molar mass of the grafted polymer chains.

This last value can be obtained by gel permeation chromatography (GPC), which will give a molar mass distribution. There are two ways to acquire free polymer chains for GPC studies; the first method is cleavage of the brush from the substrate. This method requires as first a linker between the substrate and the chain that can be cleaved. Most of the time a strong acid is used for the cleavage, which can cause undesired side reactions. The other requirement is that a large surface area is functionalized with polymer brush in order to have enough material for GPC analysis. The second option to obtain the free polymer chains is the use of a sacrificial initiator in the polymerization 
mixture. Also this method has some drawbacks as bulk polymerization often cannot be compared with a surface-initiated polymerization, since for the latter, confinement effects and substrate geometries play a role.

A more recent and sophisticated experiment to determine grafting density is reported by Kutnyanszky et al.. A scheme of their performed measurement is displayed in Figure $2.11 .^{38}$

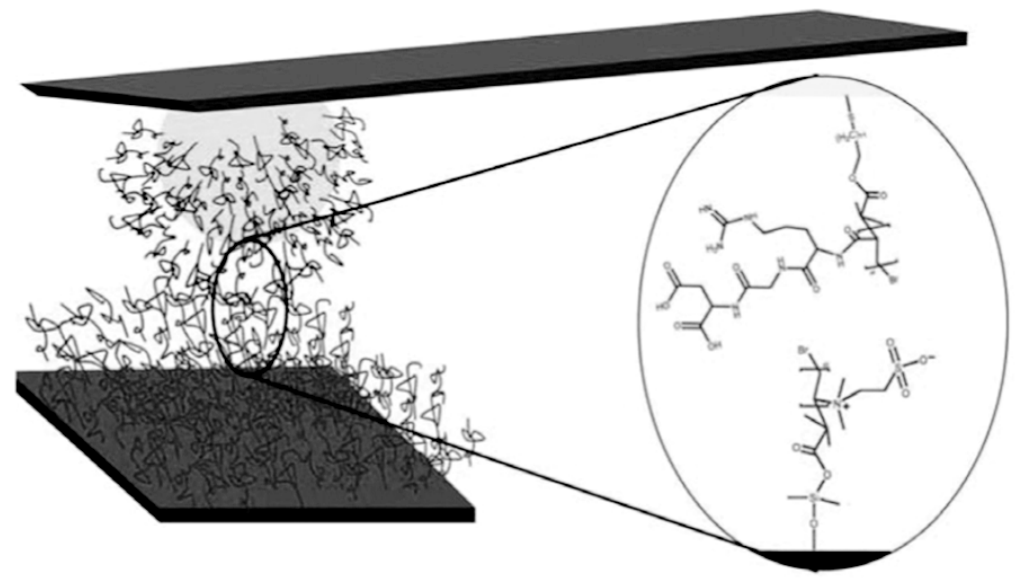

Figure 2.11: Schematic presentation of a zwitterionic polymer brush functionalized AFM colloidal probe brought in contact with a weak polyelectrolyte brush functionalized planar surface. Reprinted with permission from reference 38. Copyright 2012 Elsevier.

The grafting density could be calculated from data of AFM based colloidal probe compression measurements. A planar Si surface was functionalized with a zwitterionic polymer brush and a weak polyelectrolyte brush was grafted from gold colloidal probes (as depicted in Figure 2.11). The grafted surfaces were probed against unmodified surfaces and against each other. The obtained force-distance approach curves were processed with fits based on the model of de Gennes. ${ }^{38}$

\section{5 (Stimulus responsive) polymer brushes in pores for controlled molecular transport} or ion permeation

Grafting stimulus responsive polymer brushes onto and from porous platforms offers opportunities to different fields including delivery systems, lab-on-a-chip, micro- and nanofluidics and (bio)molecular screening. ${ }^{39}$ In this paragraph first work of research groups who reported in several papers on this topic are discussed. Work of Ito, Imanishi et al. is discussed first, work of Yameen, Azzaroni et al. will follow and at last there is 
work of Tagliazucchi, Szleifer et al. The last part of this paragraph presents remaining examples.

Ito, Imanishi et al. were one of the first who brought up chemical gating by smart polymer grafts covalently tethered on porous membranes. In 1989 they reported on an insulin releasing system in response to glucose. ${ }^{40}$ The principle of their controlled release system of insulin is showed in Figure 2.12 .

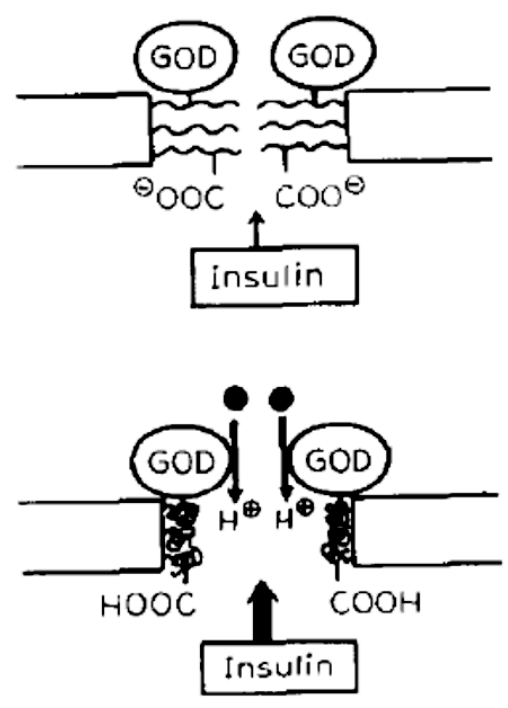

0: glucose

Figure 2.12: above) PAA grafts are deprotonated, charged and extended; insulin is not available. below) PAA graft are protonated, uncharged and collapsed; insulin is released. Reprinted with permission from reference 40. Copyright 1989 Elsevier.

The system of Ito et al. shown in Figure 2.12 is based on the conformational changes of the poly(acrylic acid) (PAA) grafts in response to $\mathrm{pH}$. Ito et al. immobilized the enzyme glucose oxidase (GOD) to the PAA brush functionalized membrane. At neutral pH, when there is no glucose, the carboxylic acid groups are deprotonated, and therefore charged and extended. By adding glucose to the system, the carboxylic acid groups are protonated; since GOD will oxidize the glucose to gluconic acid, which in turn is responsible for the protonation. The result is uncharged PAA chains, which collapse to coils and open the pores of the membrane for insulin. ${ }^{40}$ 
Later Ito, Imanishi et al. reported on other $\mathrm{pH}$ sensitive and oxidoreduction sensitive (bio)polymer brushes, which were used to regulate the liquid flow rate through porous membranes. ${ }^{41-44}$ Their work presented control of water permeation by $\mathrm{pH}$ and ionic strength and they also took into account the degree of polymerization and the grafting density. Ito et al. also reported on the self-assembly of pH-responsive (bio)polymer grafts on gold-coated membranes for controlled transport. ${ }^{45,46}$ In the years after stimulus responsive gating by polymer brushes developed and by the year 2000 several membrane devices could be reviewed, also responses to temperature and photoirradiation were included. ${ }^{47}$

Later, around the year 2008, the idea of grafting polyelectrolytes from silicon-based nanopores was picked up by Yameen, Azzaroni et al. for proton conducting membranes as an alternative for perfluorinated polyelectrolytes. In their work they graft a polyelectrolyte brush by SI-ATRP from a macroporous silicon membrane, as shown in Figure 2.13.

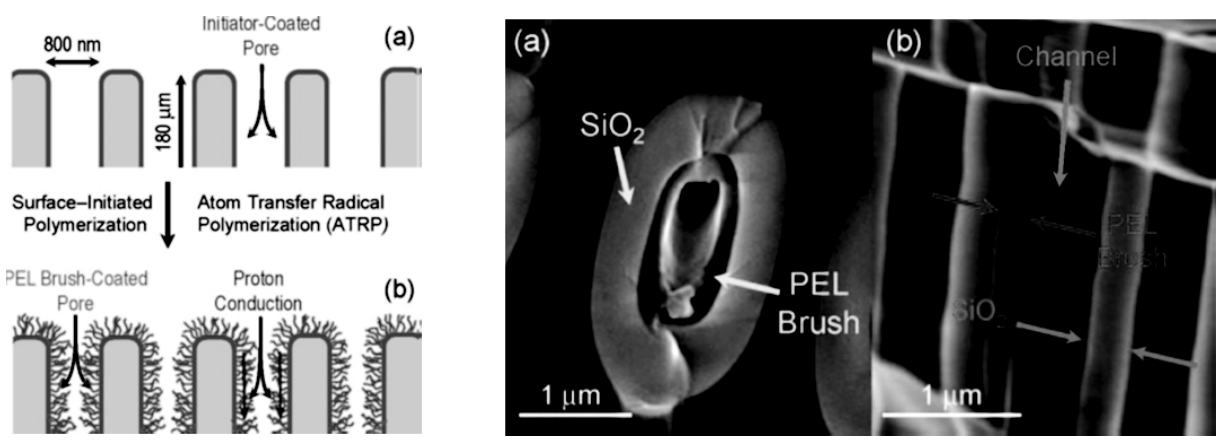

Figure 2.13: Left) A macroporous silicon membrane functionalized with a initiator-terminated self-assembled monolayer (a) is immersed in the ATRP mixture for grafting of the polyelectrolyte chains (b). Right) SEM images of a functionalized nanopore: (a) cross-section and (b) longitudinal cross-section. Reprinted with permission from reference 48. Copyright 2008 American Chemical Society.

The work of Yameen et al. on proton conducting membranes elaborates upon the use of copolymer brushes and other polyelectrolyte brushes. ${ }^{49,50}$

Yameen, Azzaroni et al. also looked more into manipulating ionic transport with stimulus responsive polymer brushes grafted from single solid-state nanopores to mimic ion channels of biological membranes. They used a zwitterionic polymer brush to tune the ionic transport by $\mathrm{pH}$, a thermoresponsive brush to tune gating by temperature and 
other $\mathrm{pH}$-responsive polymer brushes to tune transport by $\mathrm{pH}$. In Figure 2.14 an example of a $\mathrm{pH}$-responsive polymer brush is displayed. ${ }^{51-54}$

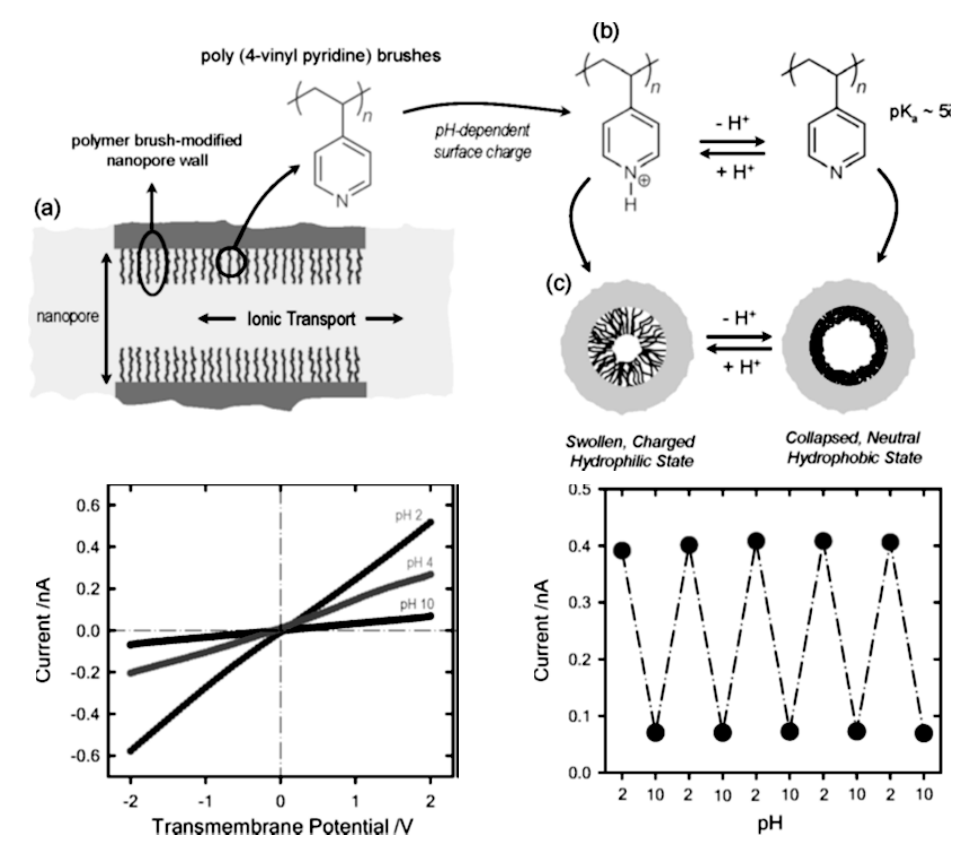

Figure 2.14: Above) Schematic of functionalized nanopore (a), pH-responsive behavior of grafted polymer (b) and conformational changes inside the nanopore (c). Below) Cyclic voltammetry of a functionalized pore at varied $\mathrm{pH}$ values (left side) and the reversible switching between $\mathrm{pH} 2$ and $\mathrm{pH} 10$ of the current across the functionalized nanopore (right side). Reprinted with permission from reference 53. Copyright 2009 American Chemical Society.

Tagliazucchi, Szleifer et al. also reported several papers on solid-state nanopores functionalized with stimulus responsive polymers with an emphasis on polyelectrolyte brushes. Their work mostly contains theoretical studies, sometimes compared with experimental observations, to understand fundamentally the responsive behavior of soft matter in confined spaces. When polyelectrolyte brushes are taken into consideration, a $\mathrm{pH}$-dependent ionic conductivity through the acid-base equilibrium and the additional charges is predicted, which has the same outcome as experimental observations. It could also be predicted that the dissociation constant of the polyelectrolyte brushes are depending on the geometries of the confined space. A comprehensive theoretical study was performed to investigate the conformation of grafted polymer layers in nanopores as a function of solvent quality and pore geometry. Pore radius, pore length and the 
grafting position along the pore play important roles in the conformation. This molecular organization of the grafted polyelectrolyte chains from the nanopores influences the ionic transport across the channels. These theoretical studies contribute to a better design of stimulus responsive polymer brush functionalized nanopores for controlled molecular gating. In Figure 2.15 a schematic and modulations of the studies are shown. ${ }^{55-58}$

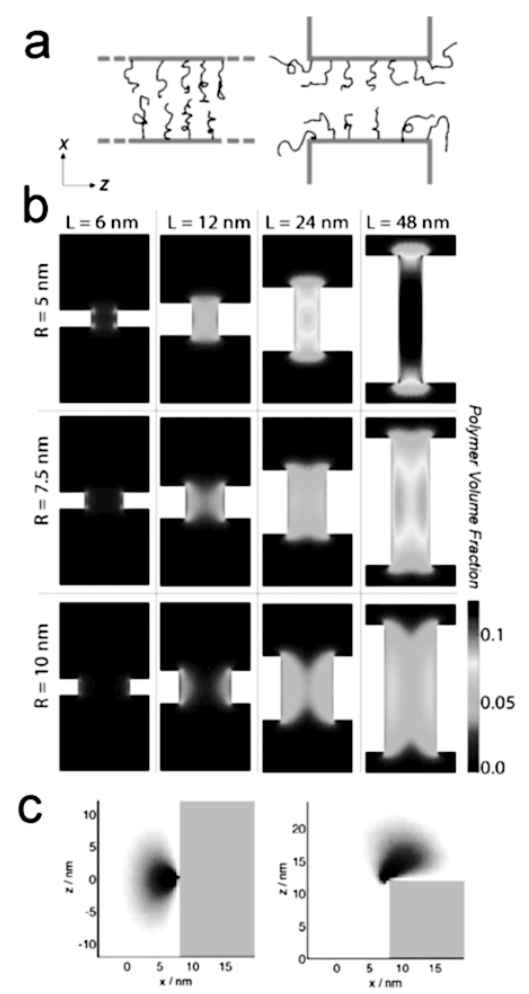

Figure 2.15: a) Schematic representation of polymer chain organization in long pores (left) and in short pores (right). In short nanopores, the polymer chains can stretch out of the pore. b) Modulation of volume fraction of polymer segments for varied pore sizes in a good solvent (chain length and grafting density are constant). c) Projection of a single polymer chain within a polymer film. Chain is attached to the center of the pore (left) or to the edge of the pore (right). Reprinted with permission from reference 58. Copyright 2012 The Royal Society of Chemistry.

Besides the work of the three research groups presented above, there are several other groups who reported on smart membranes by responsive grafts. Peng and Cheng e.g. photografted PMAA and pNIPAM from polyethylene (PE) membranes with a variety 
of grafting densities and could tune the permeability by changing the $\mathrm{pH}$ or the temperature of the surrounding environment. ${ }^{59,60}$ Later they also reported on cografting from PE membranes of these two polymers, which resulted in a double-stimuli responsive permeability behavior. ${ }^{61}$ Ito, Kimura et al. developed a copolymer graft with two functionalities; one monomer with a LCST and the other monomer with a crown receptor. The shift in the LCST was caused by trapping of a specific ion by the crown receptor. This resulted in a molecular recognition ion gate, which can also control the permeation and solute size. ${ }^{62}$ Friebe and Ulbricht also performed a copolymerization to obtain a grafted diblock copolymer responding to two stimuli. The grafting was done by ATRP from track-etched poly(ethylene terephthalate) membranes. The grafted chains contained a $\mathrm{pH}$-responsive block and a thermoresponsive block, and by changing the environmental conditions in $\mathrm{pH}$ and temperature resulted in four different brush heights. This is shown in the schematic of Figure $2.16 .^{63}$

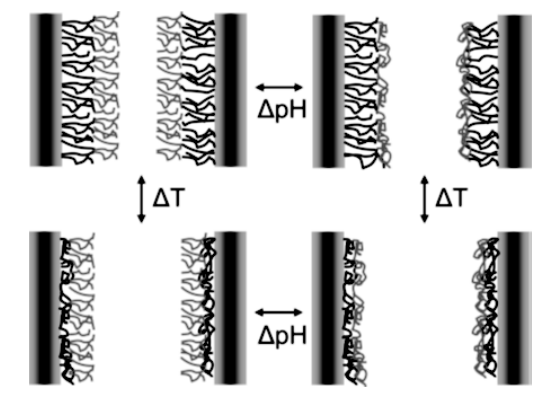

Figure 2.16: Schematic presentation of a double-stimulus polymer brush functionalized membrane. First a thermoresponsive block is grafted and subsequently a $\mathrm{pH}$-responsive block is grafted. Brush height can be tuned by both $\mathrm{pH}$ and temperature. Reprinted with permission from reference 63. Copyright 2009 American Chemical Society.

Another double responsive system is developed by Hou et al., who did not use copolymerization as the examples before, but employed asymmetric functionalization. This was done to mimic the complex channels in nature with various functions. One half of the nanochannel was functionalized with a $\mathrm{pH}$-responsive graft and the other half with a thermoresponsive graft. Therefore different ionic transport through the nanochannel could be tuned. ${ }^{64}$ 


\subsection{Characterization of stimulus responsive polymer brushes in pores}

Besides the functionalization of porous membranes with stimulus responsive brushes and investigating their applications, it is also important to characterize the polymer grafts inside the pores. This is especially of interest because in the confined space of pores, different polymerization kinetics and the accessibility of the polymer grafts for conformational change play a role. ${ }^{55,65}$ Also here Ito, Imanishi et al. were one of the first who explicitly followed the response of stimulus responsive grafts and made it visible. Ito et al. achieved this for $\mathrm{pH}$-sensitive polymer brushes by in situ AFM measurements, which is displayed in Figure 2.17. ${ }^{66,67}$

(a)

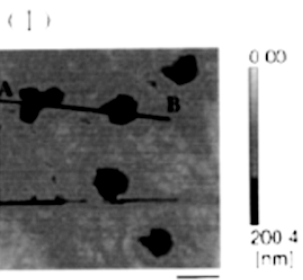

pH $=2$
(II)

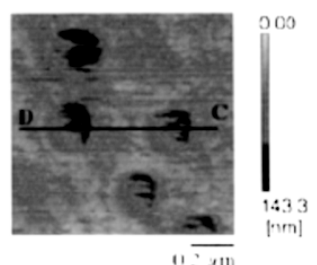

$\mathbf{p H}=7$

(b)

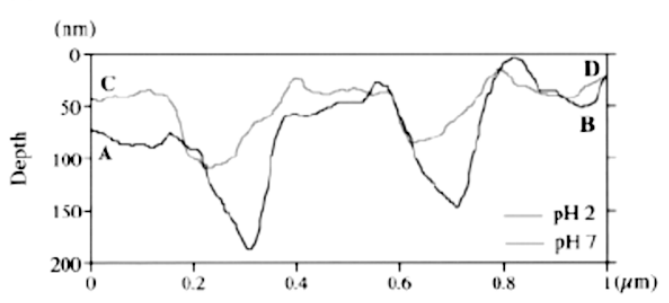

Figure 2.17: (a) AFM images of a PMMA brush functionalized membrane at (I) pH 2 and (II) pH 7.

(b) Cross-sections of the line $\mathrm{A}-\mathrm{B}$ at $\mathrm{pH} 2$ and the line $\mathrm{C}-\mathrm{D}$ at $\mathrm{pH} 7$ in (a). Reprinted with permission from reference 47. Copyright 2000 John Wiley and Sons.

In Figure 2.17 is shown, by AFM imaging, that there is a difference in polymer chain conformation between the two $\mathrm{pH}$ values. At $\mathrm{pH} 2$ the chains are collapsed and at $\mathrm{pH} 7$ the chains are extended. This results in a change in pore size between the two $\mathrm{pH}$ values, which can also be seen in the cross-sections with a lower depth at $\mathrm{pH} 7$. This example illustrates that AFM is a characterization technique that can be used to image the response of smart polymer brushes at the pore entrances. ${ }^{47}$ 
More recently, more work is reported about the specific characterization of polymer brushes inside nanopores. Although there are not that many examples, the characterization technique used in the following two examples will be again AFM, displaying its versatility. ${ }^{31}$

In the first example Lim and Deng anchored polyethylene glycol (PEG) chains to a gold nanoring on a silicon substrate. Subsequently force volume spectroscopy by AFM was performed in order to follow the reversible switching between a collapsed and extended state of the PEG brush. The conformational change of the PEG brush was induced by switching between poor and good solvent conditions, which is shown in Figure $2.18 .{ }^{68}$
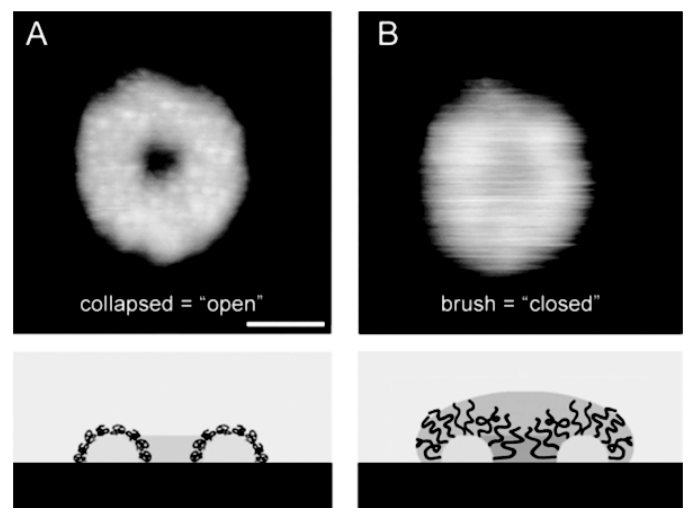

Figure 2.18: A) PEG chains in collapsed state at gold nanoring, poor solvent conditions. B) PEG chains in extended state at gold nanoring, good solvent conditions. Above contact AFM images are presented and below schematic cross-sections. Reprinted with permission from reference 68. Copyright 2009 American Chemical Society.

Lim and Deng studied in detail the interaction forces and morphological changes of a polymer graft functionalized nanopore. Their work contributes to a better understanding of the responsive behavior of polymer chains grafted from nanopores, where designing membranes for molecular gating can benefit from. ${ }^{68}$

The second and last example of characterizing stimulus responsive grafts is by Radji et al., who grafted pNIPAM brushes in a track-etched membrane. By a cryo-microtomed cut, the inner part of the functionalized membrane was exposed and AFM force spectroscopy was performed. The obtained force curves were fitted to determine the grafting density and to estimate the chain length. Their experiments displayed that the grafting density for brushes within $80 \mathrm{~nm}$ pores was ten times less than for $330 \mathrm{~nm}$ 
pores. It also appeared that the chain length for the same polymerization time was shorter for the smaller pores. ${ }^{69-71}$

\subsection{References}

1 Milner, S. T. Science 1991, 251, 905-914.

2 Zhao, B.; Brittain, W. J. Prog. Polym. Sci. 2000, 25, 677-710.

3 Polymer Brushes: Synthesis, Characterization, Applications; Advincula, R. C.; Brittain, W. J.; Caster, K. C.; Rühe, J., Eds.; Wiley-VCH: Weinheim, 2004.

4 Wu, T.; Efimenko, K.; Genzer, J. J. Am. Chem. Soc. 2002, 124, 9394-9395.

5 Bhat, R. R.; Tomlinson, M. R.; Wu, T.; Genzer, J. In Surface-Initiated Polymerization II; Jordan, R., Ed.; Springer, 2006; Vol. 198, p 51-124.

$6 \quad$ Alexander, S. J. Phys. 1977, 38, 983-987.

7 de Gennes, P. G. Adv. Colloid Interface Sci. 1987, 27, 189-209.

$8 \quad$ Milner, S. T.; Witten, T. A.; Cates, M. E. Macromolecules 1988, 21, 2610-2619.

9 Murat, M.; Grest, G. S. Macromolecules 1989, 22, 4054-4059.

10 Stuart, M. A. C.; Huck, W. T. S.; Genzer, J.; Muller, M.; Ober, C.; Stamm, M.; Sukhorukov, G. B.; Szleifer, I.; Tsukruk, V. V.; Urban, M.; Winnik, F.; Zauscher, S.; Luzinov, I.; Minko, S. Nat. Mater. 2010, 9, 101-113. Chan, A.; Orme, R. P.; Fricker, R. A.; Roach, P. Adv. Drug Delivery Rev. 2013, 65, 497-514. Nagase, K.; Kobayashi, J.; Okano, T. J. R. Soc., Interface 2009, 6, S293-S309.

13 Benetti, E. M.; Zapotoczny, S.; Vancso, G. J. Adv. Mater. 2007, 19, 268-271.

14 Alarcon, C. D. H.; Farhan, T.; Osborne, V. L.; Huck, W. T. S.; Alexander, C. J. Mater. Chem. 2005, 15, 2089-2094.

15 Minko, S.; Muller, M.; Motornov, M.; Nitschke, M.; Grundke, K.; Stamm, M. J. Am. Chem. Soc. 2003, 125, 3896-3900.

Kuroki, H.; Tokarev, I.; Minko, S. In Annual Review of Materials Research; Clarke, D. R., Ed.; Annual Reviews: Palo Alto, 2012; Vol. 42, p 343-372.

17 Zhou, F.; Biesheuvel, P. M.; Chol, E. Y.; Shu, W.; Poetes, R.; Steiner, U.; Huck, W. T. S. Nano Lett. 2008, 8, 725-730.

Dong, R.; Lindau, M.; Ober, C. K. Langmuir 2009, 25, 4774-4779.

Baum, M.; Brittain, W. J. Macromolecules 2002, 35, 610-615.

Husseman, M.; Malmstrom, E. E.; McNamara, M.; Mate, M.; Mecerreyes, D.; Benoit, D. G.; Hedrick, J. L.; Mansky, P.; Huang, E.; Russell, T. P.; Hawker, C. J. Macromolecules 1999, 32, 1424-1431. 
Barbey, R.; Lavanant, L.; Paripovic, D.; Schuwer, N.; Sugnaux, C.; Tugulu, S.; Klok, H. A. Chem. Rev. 2009, 109, 5437-5527.

Matyjaszewski, K.; Xia, J. H. Chem. Rev. 2001, 101, 2921-2990.

Luzinov, I.; Minko, S.; Tsukruk, V. V. Soft Matter 2008, 4, 714-725.

Ducker, R.; Garcia, A.; Zhang, J. M.; Chen, T.; Zauscher, S. Soft Matter 2008, 4, 17741786.

di Lena, F.; Matyjaszewski, K. Prog. Polym. Sci. 2010, 35, 959-1021.

Pyun, J.; Kowalewski, T.; Matyjaszewski, K. Macromol. Rapid Commun. 2003, 24, 10431059.

Milner, S. T.; Witten, T. A.; Cates, M. E. Europhys. Lett. 1988, 5, 413-418.

Sui, X.; Zapotoczny, S.; Benetti, E. M.; Schon, P.; Vancso, G. J. J. Mater. Chem. 2010, 20, 4981-4993.

Zhang, J. M.; Nylander, T.; Campbell, R. A.; Rennie, A. R.; Zauscher, S.; Linse, P. Soft Matter 2008, 4, 500-509.

Peter, M.; Hempenius, M. A.; Kooij, E. S.; Jenkins, T. A.; Roser, S. J.; Knoll, W.; Vancso, G. J. Langmuir 2004, 20, 891-897.

Kooij, E. S.; Sui, X. F.; Hempenius, M. A.; Zandvliet, H. J. W.; Vancso, G. J. J. Phys. Chem. B 2012, 116, 9261-9268.

Chen, T.; Amin, I.; Jordan, R. Chem. Soc. Rev. 2012, 41, 3280-3296.

Benetti, E. M.; Reimhult, E.; de Bruin, J.; Zapotoczny, S.; Textor, M.; Vancso, G. J. Macromolecules 2009, 42, 1640-1647.

Farhan, T.; Azzaroni, O.; Huck, W. T. S. Soft Matter 2005, 1, 66-68.

Kutnyanszky, E.; Vancso, G. J. Eur. Polym. J. 2012, 48, 8-15.

Adiga, S. P.; Brenner, D. W. J. Funct. Biomater. 2012, 3, 239-256.

Ito, Y.; Casolaro, M.; Kono, K.; Imanishi, Y. J. Controlled Release 1989, 10, 195-203.

Ito, Y.; Inaba, M.; Chung, D. J.; Imanishi, Y. Macromolecules 1992, 25, 7313-7316.

Ito, Y.; Nishi, S.; Park, Y. S.; Imanishi, Y. Macromolecules 1997, 30, 5856-5859.

Ito, Y.; Ochiai, Y.; Park, Y. S.; Imanishi, Y. J. Am. Chem. Soc. 1997, 119, 1619-1623.

Park, Y. S.; Ito, Y.; Imanishi, Y. Chem. Mater. 1997, 9, 2755-2758.

Ito, Y.; Park, Y. S.; Imanishi, Y. Langmuir 2000, 16, 5376-5381.

Zhang, H. J.; Ito, Y. Langmuir 2001, 17, 8336-8340.

Ito, Y.; Park, Y. S. Polym. Adv. Technol. 2000, 11, 136-144.

Yameen, B.; Kaltbeitzel, A.; Langner, A.; Duran, H.; Mueller, F.; Goesele, U.; Azzaroni, O.; Knoll, W. J. Am. Chem. Soc. 2008, 130, 13140-13144.

49 Yameen, B.; Kaltbeitzel, A.; Langer, A.; Mueller, F.; Goesele, U.; Knoll, W.; Azzaroni, O. Angew. Chem., Int. Ed. 2009, 48, 3124-3128. 
50 Yameen, B.; Kaltbeitzel, A.; Glasser, G.; Langner, A.; Mueller, F.; Goesele, U.; Knoll, W.; Azzaroni, O. ACS Appl. Mater. Interfaces 2010, 2, 279-287.

51 Yameen, B.; Ali, M.; Neumann, R.; Ensinger, W.; Knoll, W.; Azzaroni, O. Small 2009, 5, 1287-1291.

52 Yameen, B.; Ali, M.; Neumann, R.; Ensinger, W.; Knoll, W.; Azzaroni, O. J. Am. Chem. Soc. 2009, 131, 2070-2071.

53 Yameen, B.; Ali, M.; Neumann, R.; Ensinger, W.; Knoll, W.; Azzaroni, O. Nano Lett. 2009, 9, 2788-2793. Yameen, B.; Ali, M.; Neumann, R.; Ensinger, W.; Knoll, W.; Azzaroni, O. Chem. Commun. 2010, 46, 1908-1910.

Peleg, O.; Tagliazucchi, M.; Kroeger, M.; Rabin, Y.; Szleifer, I. ACS Nano 2011, 5, 4737 4747. Tagliazucchi, M.; Azzaroni, O.; Szleifer, I. J. Am. Chem. Soc. 2010, 132, 12404-12411. Tagliazucchi, M.; Rabin, Y.; Szleifer, I. J. Am. Chem. Soc. 2011, 133, 17753-17763. Tagliazucchi, M.; Szleifer, I. Soft Matter 2012, 8, 7292-7305. Peng, T.; Cheng, Y. L. J. Appl. Polym. Sci. 1998, 70, 2133-2142. Peng, T.; Cheng, Y. L. Polymer 2001, 42, 2091-2100. Ito, T.; Hioki, T.; Yamaguchi, T.; Shinbo, T.; Nakao, S.; Kimura, S. J. Am. Chem. Soc. 2002, $124,7840-7846$. Friebe, A.; Ulbricht, M. Macromolecules 2009, 42, 1838-1848. Hou, X.; Yang, F.; Li, L.; Song, Y. L.; Jiang, L.; Zhu, D. B. J. Am. Chem. Soc. 2010, 132, 11736-11742. Ito, Y.; Kotera, S.; Inaba, M.; Kono, K.; Imanishi, Y. Polymer 1990, 31, 2157-2161. Ito, Y.; Park, Y. S.; Imanishi, Y. J. Am. Chem. Soc. 1997, 119, 2739-2740. Ito, Y.; Park, Y. S.; Imanishi, Y. Macromol. Rapid Commun. 1997, 18, 221-224. Lim, R. Y. H.; Deng, J. ACS Nano 2009, 3, 2911-2918.

69 Radji, S.; Alem, H.; Demoustier-Champagne, S.; Jonas, A. M.; Cuenot, S. Macromol. Chem. Phys. 2012, 213, 580-586. Alem, H.; Jonas, A. M.; Demoustier-Champagne, S. Polym. Degrad. Stab. 2010, 95, 327331.

71 Alem, H.; Duwez, A. S.; Lussis, P.; Lipnik, P.; Jonas, A. M.; Demoustier-Champagne, S. J. Membr. Sci. 2008, 308, 75-86. 


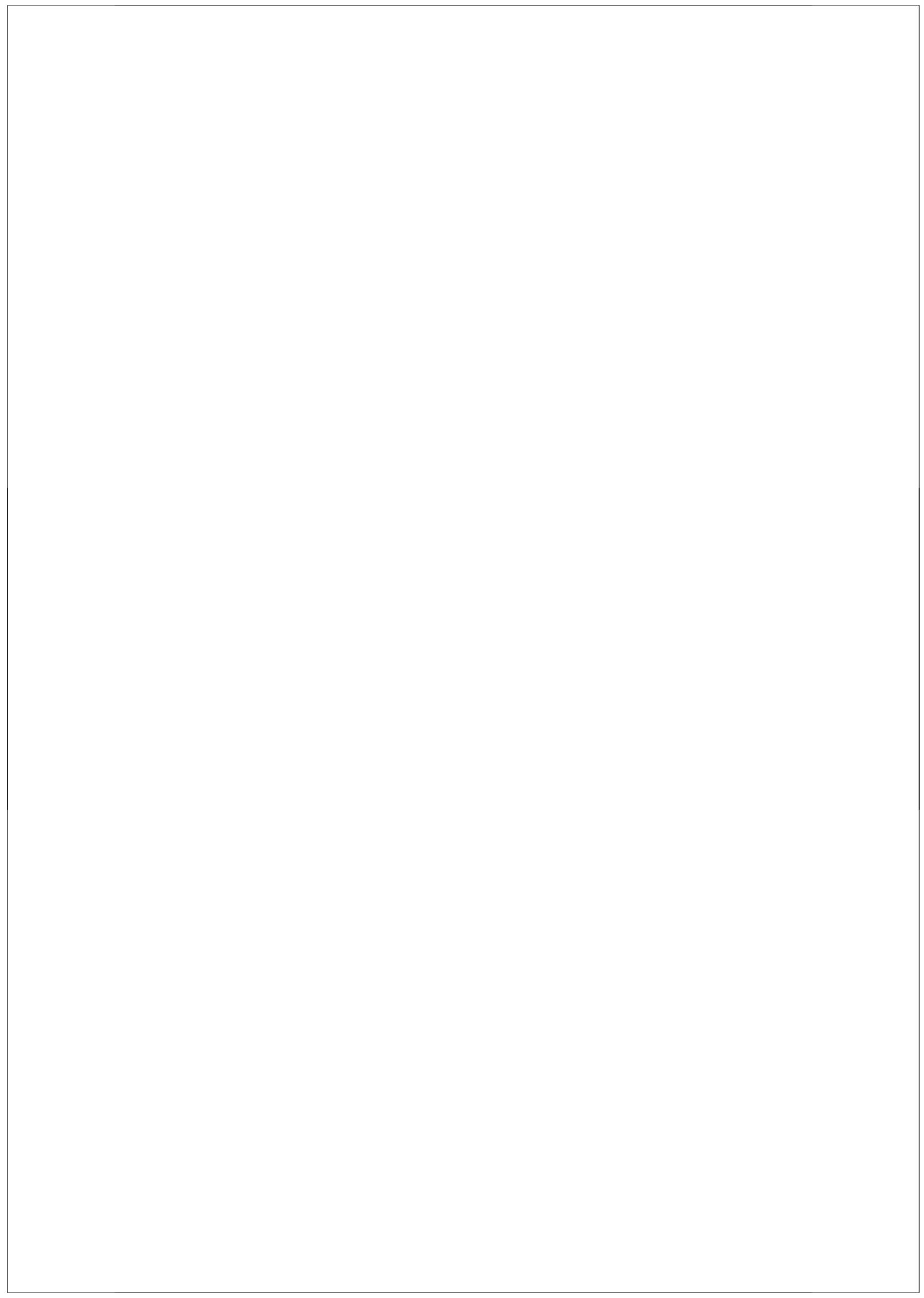




\section{Chapter 3}

\section{Pore-spanning lipid bilayers for membrane protein assays}

In the previous chapter a general literature overview is presented about stimulus responsive polymer brushes and their application for the functionalization of porous surfaces. This chapter contains a short overview of the state-of-the-art of pore-spanning lipid bilayers for membrane protein assays. The issues described include, in addition to the nanopore functionalization with a stimulus responsive polymer brush to control transport of ions and drug candidates (generally discussed in Chapter 2), the design of polymer brush architectures to support lipid bilayer membranes with integrated membrane proteins on nanoporous surfaces. This chapter presents the literature basis for the work in Chapter 6, and combined with Chapter 2 it presents the whole literature background on which the work in this thesis is based.

* Parts of this chapter will be submitted for publication by Santonicola, M.G.; de Groot, G.W.;

Vancso, G.J. 


\subsection{Functional assays for membrane proteins}

Drug targets are cellular and molecular structures in the human body, which can be influenced by drugs. In 2011, 435 drug targets were identified and about 55\% of these targets were membrane proteins. As there are around 7500 different membrane proteins in humans, this displays the importance for the development of functional assays for screening of these proteins. ${ }^{1-3}$ These screening tools will provide a more fundamental knowledge on the structure-function relationship of membrane proteins and therefore will hasten the development of new drugs.

The current assays rely in the case of ligand-binding assays on fluorescent recording of labels after a binding event, whereby a label can affect the biochemical behavior of the membrane proteins. In addition, these assays, together with the patch-clamp based methods, suffer from low throughput and thus low information content. Therefore, future screening assays should be ideally label-free and high-throughput, and facilitate the testing of low-molecular-weight drug candidates.

Membrane proteins are integrated in the walls of biological cells and cellular compartments, a scheme of which is presented in Figure 3.1. Therefore, the first concepts for functional assays were directed to the development of methods and platforms for the formation of artificial lipid bilayers. These artificial lipid bilayers should have properties as close as possible to those of natural lipid bilayers, as the integration of membrane proteins in these structures should not cause any conformational changes and/or denaturation of the proteins. Besides methods and platforms, also several characterization techniques were developed specifically for artificial lipid bilayers.

In section 3.2 a literature overview of the formation and characterization of artificial lipid bilayers is presented with a focus on polymer-supported systems and porespanning lipid bilayers. The final goal of the ASMENA project was the integration of membrane proteins into artificial lipid bilayers for screening of these proteins with respect to pharmaceuticals. This is discussed in more detail in section 3.3, again with emphasis on free-standing lipid bilayers on nanoporous supports. 


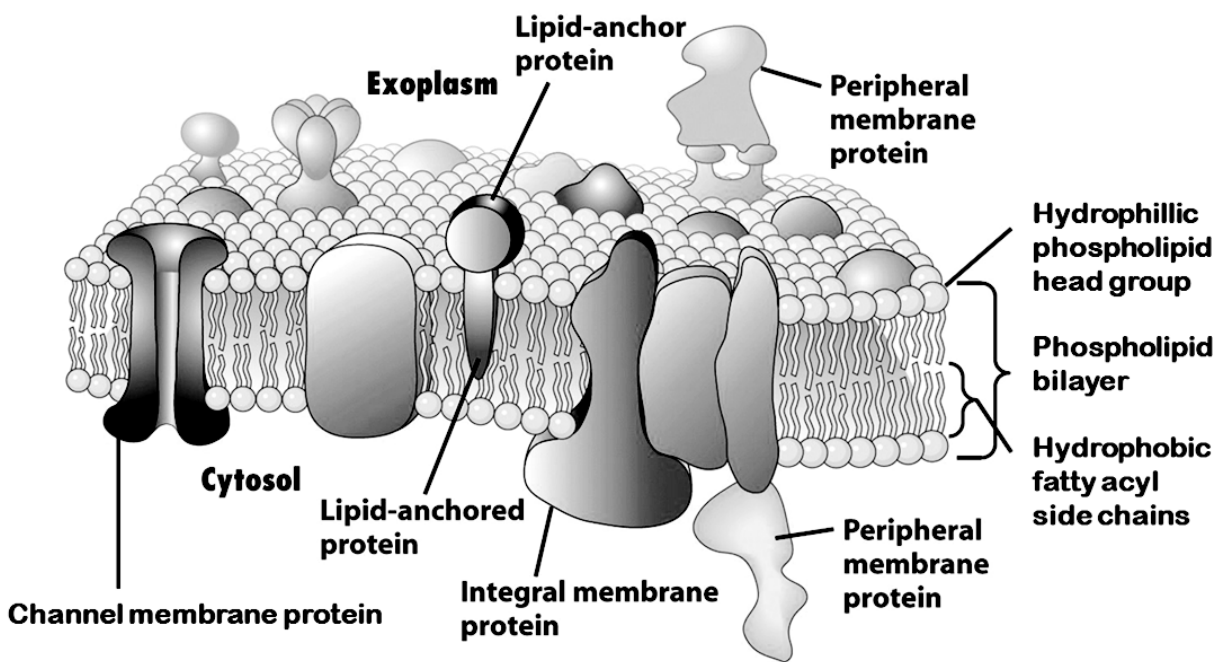

Figure 3.1: Schematic illustration of a cell membrane. Proteins are embedded in a matrix of phospholipid molecules. Several other membrane constituents are present in the natural lipid bilayer. Adapted from reference 4. Copyright 2007 W. H. Freeman \& Co Ltd.

\subsection{Supported and pore-spanning artificial lipid bilayers}

Artificial lipid bilayers for the study of membrane proteins offer several advantages. First, the system is more controlled than when natural membranes are used, in which the lipid composition is unknown and it is difficult to distinguish the membrane protein of interest from other proteins present.

There are two configurations generally occurring for artificial lipid bilayers: the first are the so-called supported lipid bilayers, where the lipid membranes are formed on solid supports. The second configuration is the pore-spanning lipid bilayers, also called free-standing lipid bilayers or suspended lipid bilayers. Both configurations are widely used and investigated, and have their advantages and disadvantages.

In section 3.2.1 the formation and characterization of artificial lipid bilayers will be discussed for both configurations. Some examples of supported lipid bilayers on responsive polymers will be discussed in section 3.2.2. The following section 3.2.3 will mention details of pore-spanning lipid bilayers, the preferred configuration for screening of membrane proteins. In the last section 3.2.4, few examples will be presented where nanoporous platforms are functionalized with polymers to suspend lipid bilayers over a single pore or an array of them. 


\subsubsection{Formation and characterization of artificial lipid bilayers}

There are several methods to form artificial lipid bilayers on solid and porous supports. Lipid bilayers formed on solid supports are prepared without organic solvent, with the advantage that residual aliphatic molecules cannot affect the structure of integrated membrane proteins and therefore the functionality of the proteins.

Spontaneous vesicle rupture of liposomes (Figure 3.2.a1) or a giant unilamellar vesicle (GUV) can be promoted by choosing the appropriate surface. A variety of tethered systems (Figure 3.2.a2) and cushioned systems are also developed, which also initiate the rupture of liposomes. ${ }^{2,5}$ Other advantages of cushioned systems are discussed in section 3.2.2.
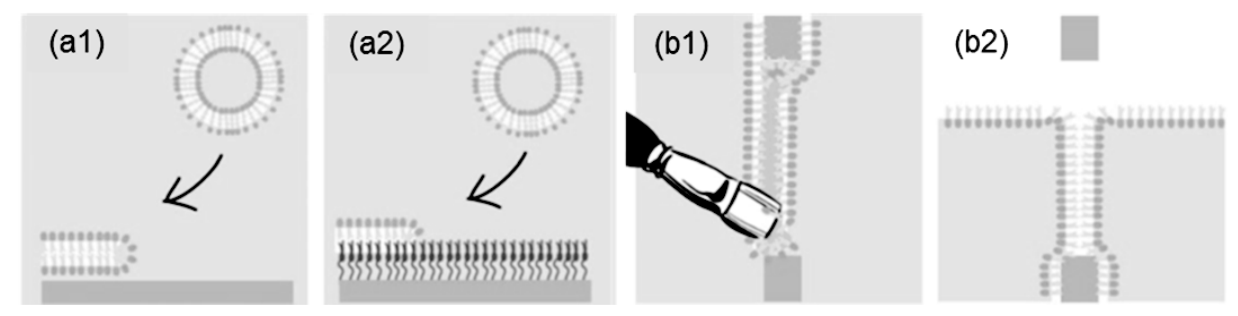

Figure 3.2: Formation of lipid bilayers on solid supports (a) and on porous supports (b): (a1) promoted vesicle rupture, (a2) promoted vesicle rupture on tethered monolayer, (b1) painting of lipids, (b2) Langmuir-Blodgett technique. Adapted with permission from reference 5. Copyright 2011 The Royal Society of Chemistry.

The first method that was developed to obtain a free-standing artificial lipid bilayer was the painting of lipids dissolved in an organic solvent over an opening (Figure 3.2.b1), so-called black lipid membranes. As stated above, a disadvantage of this method is the use of an organic solvent, but also the lifetime of these lipid bilayers is only a few hours. A second method, without the use of organic solvent, is the Langmuir-Blodgett technique (Figure 3.2.b2). In this technique a lipid monolayer is formed at an air/water interface and the water level is raised along an opening to form a lipid bilayer. Nevertheless, it is difficult to obtain defect-free lipid bilayers with this technique and also the short lifetime is a disadvantage. ${ }^{5}$ Another method to form a solvent-free suspended lipid bilayer is directing a GUV to a pore opening followed by a direct rupture into a lipid bilayer. ${ }^{6}$ More recently, the direct rupture of liposomes was demonstrated also over nanopores. ${ }^{7,8}$ A last method developed for the formation of free-standing lipid bilayers is forming the membrane on a solid support and driving it with shear force over 
pores. $^{9}$ For this last method microfluidics and lab-on-a-chip techniques play an important role. ${ }^{10-13}$

Characterization of formed lipid bilayers can be done with a variety of techniques. Two of them that are widely used and complement each other are electrochemical impedance spectroscopy (EIS) and fluorescence recovery after photobleaching (FRAP). EIS investigates the formed lipid bilayer by electrochemistry, which results in a membrane resistance and a capacitance. These results display if a lipid bilayer is complete and has good sealing properties, or if there are defects, which will make it impossible to measure protein activity. For supported lipid bilayers, the substrate functions as the working electrode, whereas for free-standing lipid bilayers the two electrodes can be placed on both sides of the lipid bilayer. FRAP is an optical technique that is used to determine the fluidity of the formed lipid bilayer. For FRAP measurements lipid molecules are mixed with two to three percent of fluorescent dye molecules. After lipid bilayer formation a small area is bleached under a confocal microscope and the recovery is followed over time. From the time for a full recovery a diffusion coefficient can be calculated, which indicates the functionality of the formed lipid bilayer.

Other characterization techniques include those that can follow vesicle deposition, vesicle rupture and lipid bilayer assembling, examples are surface plasmon resonance (SPR), dual polarization interferometry (DPI), quartz crystal microbalance with dissipation monitoring (QCM-D) and optical waveguide light spectroscopy $(\mathrm{OWLS})^{14}$. Imaging lipid bilayers with atomic force microscopy (AFM) is used to investigate topography and homogeneity. Probing the elastic properties of free-standing lipid bilayers by indentation with a tip can also be achieved by AFM, such as in the work of Janshoff et al. ${ }^{15-18}$

Another way to determine the functionality of an artificial lipid bilayer is the selfintegration of peptides into the membrane and record a current through these model peptides by electrochemical methods. If the activity of peptidic ion channels can be monitored it means that the formed lipid bilayer is sealing and virtually defect-free. The reason why these peptides are so commonly used is that their structure, handling and fragility is far less complicated than that of membrane proteins. ${ }^{2}$ The integration of membrane proteins into artificial lipid bilayers will be discussed in section 3.3. 


\subsubsection{Supported lipid bilayers by stimulus responsive polymers}

Mimicking a natural lipid bilayer can be accomplished by forming an artificial lipid bilayer on a solid support. One of the major advantages of the presence of a solid support is the long-term stability of the lipid bilayer, whereas the life time of free-standing lipid bilayers is generally limited to few hours. Assembly of the lipid bilayer directly on the solid support is possible, but it is preferred to have for instance a thin polymer layer in between the support and the lipid bilayer to mimic the extracellular matrix. ${ }^{19}$ Using an intermediate layer brings integrated proteins further from the solid support, so that the configuration, and therefore the functionality, of the proteins is less affected by the contact. In addition, sensitivity issues during electrochemical measurements are decreased, as the ion accumulation between the lipid bilayer and the support is less. ${ }^{5}$ Thereby is patterning of these supported artificial lipid bilayers interesting to create an array with a variety of patches with a different membrane protein for screening. ${ }^{20}$

The first example of a responsive polymer-supported lipid bilayer is that of Kaufmann et al.. $^{21}$ In their work a dual-responsive copolymer cushion was used to support a cationic lipid bilayer. The copolymer consisted of a temperature-responsive and a $\mathrm{pH}$ responsive polymer block, with the goal of actively tuning the lipid bilayer characteristics. The copolymer was first synthesized via radical polymerization, and subsequently grafted onto a polymer interlayer of $6 \mathrm{~nm}$ on glass coverslips. The lipid bilayer was formed by a drying/rehydration procedure and the functionality of the obtained supported lipid bilayer was probed by fluorescence recovery after photobleaching (FRAP). The lipid mobility on the dual-responsive polymer cushion at all swelling states turned out to be constant and higher than for lipid bilayers formed directly on glass supports. The system developed by Kaufmann et al. makes it possible to tune film thickness, charge and polarity below the lipid bilayer, which in the future can be used to control ion transport through integrated membrane proteins.

Another example of a pH-responsive cushion used to support a lipid bilayer is that of El-Khouri et al. ${ }^{22}$ Their system constitutes a model system to systematically study the interactions between the membrane and the environment, like varying the conformation of the $\mathrm{pH}$-responsive cushion and $\mathrm{pH}$-responsive ion transport across the lipid bilayer. For this latter point a model peptide was incorporated in the lipid membrane. The system is shown in more detail in Figure 3.3. 


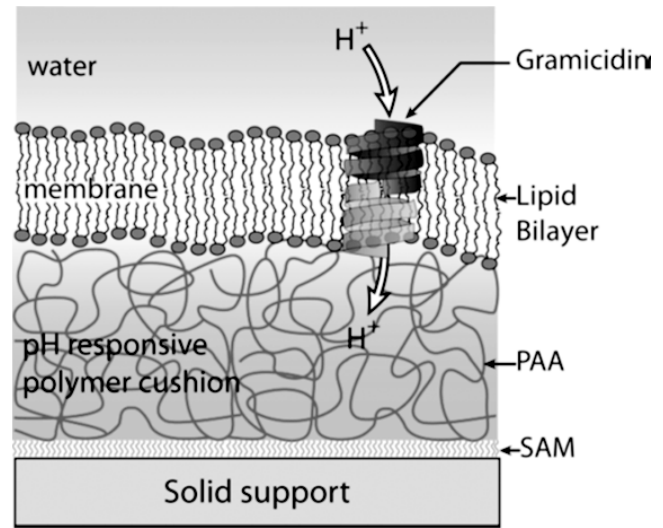

Figure 3.3: Lipid bilayer formed on a pH-responsive polymer cushion. Functionality is proven by addition of gramicidin, a self-integrating peptidic ion channel. Reprinted with permission from reference 22. Copyright 2011 American Chemical Society.

A silicon-based solid support is functionalized with an amine functional monolayer on which a lightly cross-linked poly(acrylic acid) (PAA) is spin coated. Subsequently, a lipid bilayer was formed by the Langmuir-Blodgett technique. A sealing lipid bilayer was confirmed by observing that for three hours there was no swelling of the PAA cushion when the bulk solution was cycled around the $\mathrm{pK}_{\mathrm{a}}$ of PAA. By integration of gramicidin A, a peptidic ion channel, in the lipid bilayer, the PAA cushion responded reversibly to a change in the $\mathrm{pH}$ of the bulk solution within ten minutes. These results indicate that a $\mathrm{pH}$-responsive polymer can be used for studying proton transport through membrane proteins across a lipid bilayer. ${ }^{22}$

As a final example there is work performed in our research group, in which a zwitterionic polymer brush of sulfobetaine macromolecules was grafted from several solid supports to promote rupture and fusion of neutral DOPC liposomes into a lipid bilayer. ${ }^{23}$ The adhesion of the lipid bilayer to the underlying zwitterionic polymer cushion can be tuned by varying the ionic strength of the solution.

\subsubsection{Free-standing lipid bilayers}

As mentioned before, free-standing lipid bilayers are preferred for membrane protein screening assays, in particular those involving ion channels. There are the same reasons, yet stronger, as why polymer-supported lipid bilayers are preferred over directly supported lipid bilayers, i.e. no protein contact with support and no ion accumulation 
hindering electrochemical analysis. Additionally, the accessibility of the lipid bilayer on both sides makes it possible to change conditions at only one side of the membrane.

One of the first steps in the development of membrane protein assays is the fabrication of porous supports to suspend lipid bilayers on. Progress in nanofabrication techniques helped to overcome several challenges and Han et al. managed to produce thin silicon-based nanopore arrays. ${ }^{24}$ The choice for nanoporous supports came after it was demonstrated that lipid bilayers spanned over nanopores are more stable than those spanned over micropores. Lipid bilayers were painted over the fabricated nanopores and were stable for days. Ion transport mediated by a peptidic channel could also be observed, therefore proving the functionality of the so formed lipid bilayer. ${ }^{25,26}$

Another nanoporous platform consisting of nanoporous silicon-based films for combined electrochemical and waveguide measurements was developed in the same year by Reimhult et al. ${ }^{27} \mathrm{~A}$ drawback of these platforms is that they are not accessible on both sides, as the nanoporous films are generated by etching steps on the top surface, resulting in wells.

After nanoporous platforms were established, the next step was the development of methods to form nanopore-spanning lipid bilayers without the use of organic solvents. Schmitt et al. were one of the first to report on the chemical functionalization of nanoporous platforms to promote liposome rupture into an electrically insulating porespanning lipid bilayer. A cholesterol derivative was chemisorbed to porous alumina substrates via a thiol end group, and subsequently large unilamellar vesicles (LUV) were ruptured and fused into a lipid bilayer. ${ }^{28}$ More recently, the same group reported other chemical functionalization routes to induce vesicle rupture. By varying the area functionalized by silane chemistry and the choice to use GUVs or SUVs resulted in different bilayer formations. A pore-spanning lipid bilayer was achieved by functionalization of the pore rim and rupture of GUVs. In addition, the function of the lipid bilayer as a barrier separating the underlying attoliter-sized compartments was demonstrated. This makes it possible to follow transport across the lipid bilayer. ${ }^{29,30}$

Few examples of solvent-free and free-standing lipid bilayers can be found in the literature, and most of them are already presented in section 3.2.1. The limited availability of such systems explains the need for further investigations in the development of these platforms with a focus on increasing their lifetime for use in membrane protein screening. One example is the work of Kumar et al. shown in Figure $3.4^{8}$ 
In their work a variety of parameters was investigated, including nanopore geometry, lipid membrane properties and surface interaction. The most important result was that the liposome diameter should be larger than the pore diameter to obtain a nanoporespanning lipid bilayers by liposome rupture and fusion. ${ }^{8}$ Even with this optimized parameter they only achieved $25 \%$ of spanned nanopores.
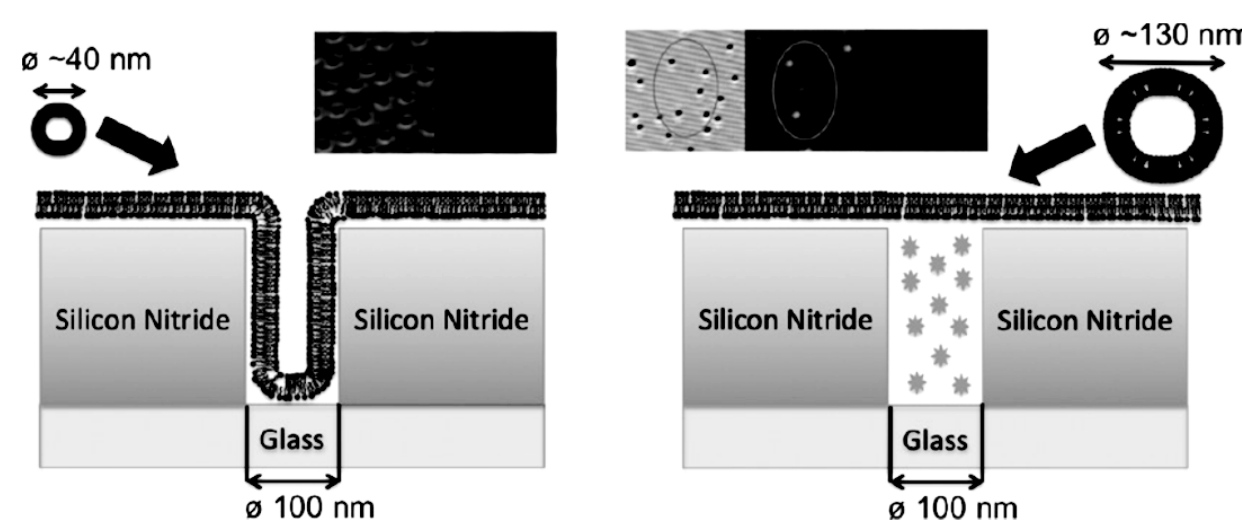

Figure 3.4: The formation of pore-spanning lipid bilayers depends on several parameters, most importantly on the size of the liposomes. Left: Liposome diameter smaller than pore diameter results in high fluorescence intensity from lipid bilayer following the pore walls. Right: Liposome diameter larger than pore diameter results in high fluorescence intensity from encapsulated dye in pore. Reprinted with permission from reference 8. Copyright 2011 American Chemical Society.

\subsubsection{Polymer-supported, pore-spanning lipid bilayers}

In the previous two sections polymer-supported and free-standing lipid bilayers were discussed. Sugihara et al. were inspired by these two approaches and combined them to form a solvent-free, pore-spanning lipid bilayer. ${ }^{31} \mathrm{~A}$ nanopore of $800 \mathrm{~nm}$ in diameter was functionalized with a polymer cushion to support a lipid bilayer spanned over the pore area. A scheme of the developed system is shown in Figure 3.5. 


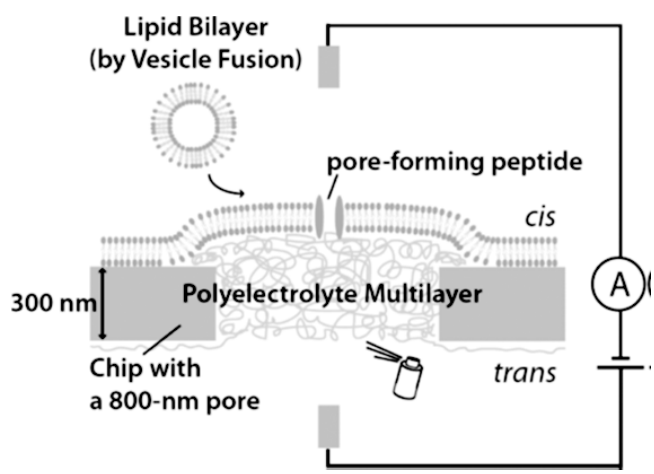

Figure 3.5: Scheme of a single silicon-based pore ( $800 \mathrm{~nm}$ in diameter) functionalized with PEM. In the next step a lipid bilayer is formed after incubation with small liposomes (50 nm in diameter). Reprinted with permission from reference 31. Copyright 2010 American Chemical Society.

A polyelectrolyte multilayer (PEM) was sprayed into the nanopore from the backside (trans), and a lipid bilayer was formed on the topside (cis) by the rupture of oppositely charged liposomes with a diameter of $50 \mathrm{~nm}$. By filling up the nanopore with the PEM these relatively small liposomes could not diffuse to the other side of the porous support, therefore forming a lipid bilayer only at the top of the platform. PEM also increased the lifetime of the giga-sealing lipid bilayer and was permeable to monovalent ions. The permeability of the lipid bilayer was investigated after integration of the poreforming peptide melittin into the membrane. The peptide channel activity could be recorded for 2.5 weeks. Previously, Schmidt et al. also suspended artificial lipid bilayers on a polymer hydrogel, only those were prepared using organic solvents. ${ }^{32,33}$

The use of pore-spanning lipid bilayers on polymer-modified supports seems to be promising, as the formed lipid bilayers are stable over several days and ions are still transported by the charged polymers without increased resistance. ${ }^{34}$

\subsection{Integration of membrane proteins in artificial lipid bilayers}

Membrane proteins fulfill different functions in the human body, as shown in Figure 3.6. Due to the fact that they are present in most human cells, receptors constitute the largest class of membrane proteins that are currently drug targets. Ion channels are important transporters in the cell membrane, and therefore also an important class of drug targets. Other transporters and enzymes are smaller classes of membrane proteins as drug targets, but still important. 


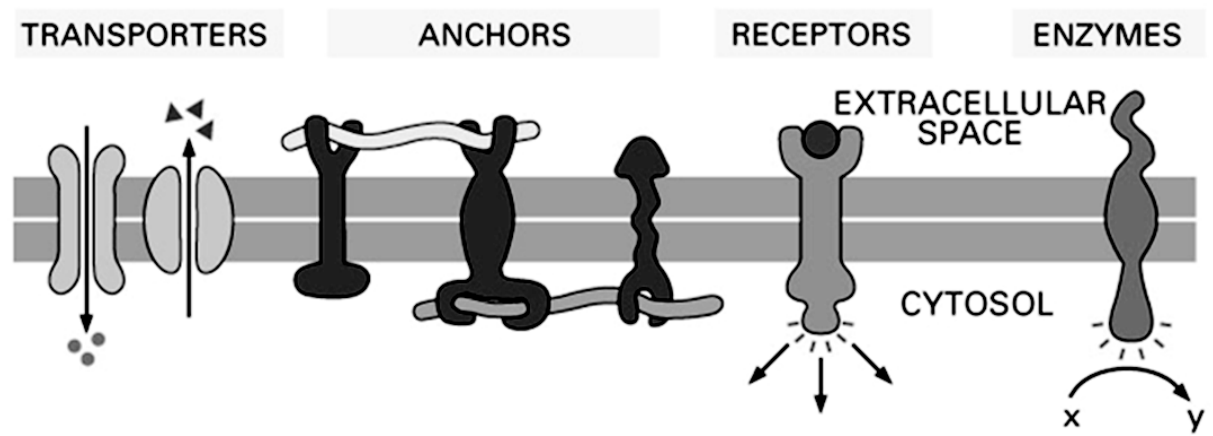

Figure 3.6: Scheme of membrane proteins as transporters, anchors, receptors and enzymes at the interface between extracellular space and cytosol. Reprinted from reference 35 . Copyright 2003 Garland Science.

The final step in the development of membrane protein assays is the integration of these proteins into artificial lipid bilayers. As mentioned in section 3.2.1, the integration of model peptide channels is an established method to prove the functionality of a lipid bilayer. Studer et al. reported the self-integration of melittin and $\alpha$-hemolysin in painted suspended lipid bilayers and that their channel activity could be monitored. ${ }^{36}$ However, most of the available work is conducted using model systems: melittin is a small peptidic ion channel, whereas $\alpha$-hemolysin is one of the few membrane proteins that inserts spontaneously in lipid bilayers. In almost all cases, pharmaceutically relevant membrane proteins have more complicated structures, and are very fragile and difficult to handle. One of the major limitations of working with these proteins is due to their amphipathic nature, with the transmembrane hydrophobic domain surrounded by hydrophilic regions, which makes their integration in functional form into artificial lipid bilayers highly challenging.

Generally, before integration of membrane proteins in artificial lipid bilayers, several purification and preparation steps are needed, including protein expression, solubilizing the proteins in buffers containing detergents and reconstitution into liposomes. Subsequently, the methods presented in Figure 3.7 can be used for the integration. 


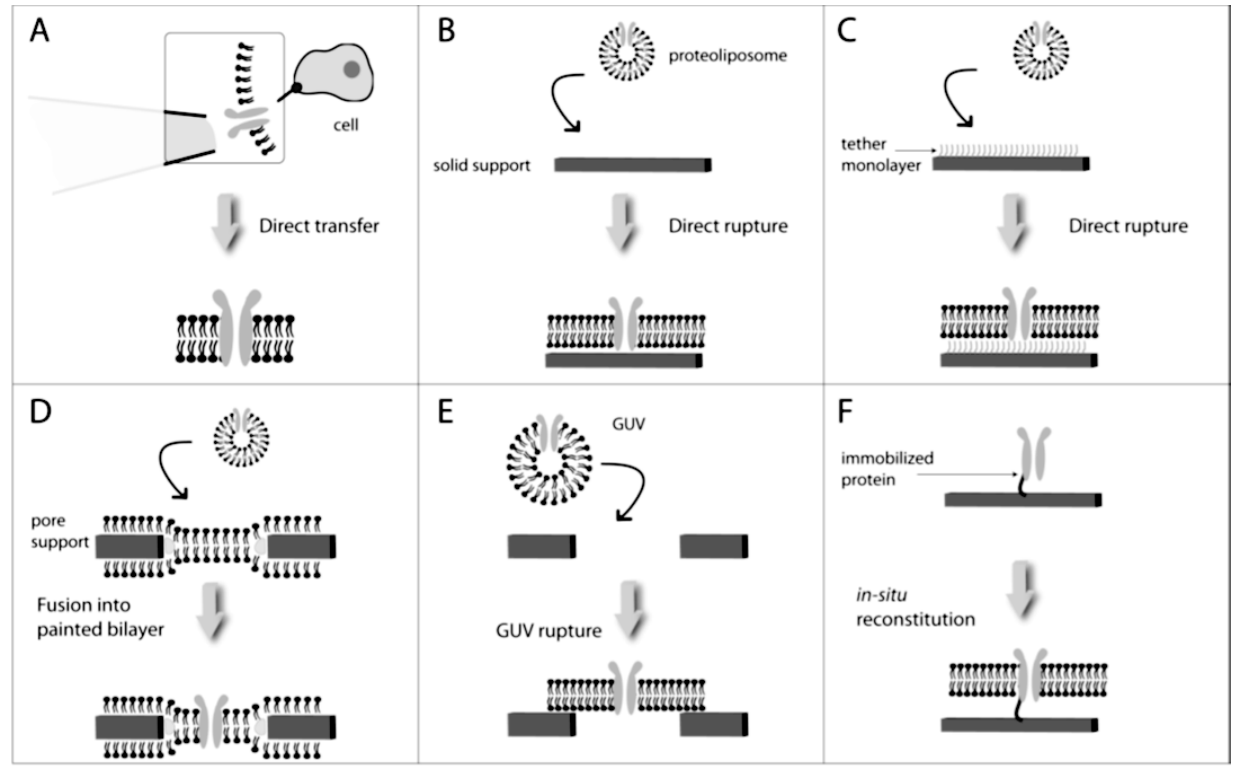

Figure 3.7: Methods to integrate membrane proteins in artificial lipid bilayers: (A) direct transfer from a natural cell membrane; (B) and (C) rupture of proteoliposomes into supported lipid bilayers on solid surfaces; (D) and (E) fusion of proteoliposomes into free-standing lipid bilayers over pores; (F) reconstitution by membrane protein binding on surface followed by a lipid bilayer formation around the protein. Reprinted from reference 2. Copyright 2012 MDPI.

Membrane proteins can be directly isolated from a cell membrane using a glass pipette (Figure 3.7A), or they can be reconstituted in lipid vesicles to form so-called proteoliposomes. The proteoliposomes can be ruptured and fused into lipid bilayers on solid supports, tethered or cushioned supports and porous supports, as shown in Figure 3.7B, 3.7C and 3.7D, respectively. An example of membrane protein systems on porous supports is from Wang et al., who integrated the transmembrane protein aquaporin in forward osmosis membranes for water reuse. ${ }^{37}$ Pore-spanning membranes were obtained by the rupture and fusion of proteoliposomes. Membranes containing aquaporin were characterized by permeability 3000 times higher than that of the membranes without membrane protein. Roder et al. also reported reconstitution of membrane proteins in polymer-supported lipid bilayers by proteoliposome rupture. ${ }^{38}$ Their device was an analytical platform, which can probe diffusion and interactions of integrated membrane proteins.

Besides proteoliposomes rupture, the fusion-in of proteoliposomes into an already established artificial lipid bilayer is also a possibility. This process can occur 
spontaneously or by mediators. Studer et al. reported the preparation and reconstitution of a bacterial voltage-gated sodium channel in a liposome. A solution of proteoliposomes was added to a painted free-standing lipid bilayer spanned over micropores. The integration of the ion channel in functional form was confirmed by recording its activity using electrochemical methods. However, this was not achieved by a preferred direct rupture of the proteoliposomes to a pore array. ${ }^{39}$

The reconstitution of membrane proteins can also be achieved using GUVs and their directed rupture can be induced by guiding the GUVs to a solid support or porous support, as shown in Figure 3.7E.

The last method for membrane protein integration is presented in Figure 3.7F in which a membrane protein is immobilized via a His-tag ${ }^{40}$ and the lipid bilayer is formed around the protein. This method can be applied to solid supports, as Friedrich et al. reported for an enzyme. ${ }^{41}$ Another possibility is to first functionalize the porous support with a His-tag. ${ }^{42}$ This method has the advantage that the orientation of the membrane proteins on surface can be controlled, and at the same time proteins can be directed above the pore openings by functionalizing only the pore walls.

In conclusion, the reconstitution of functional membrane proteins and the immobilization on solid supports, porous and non-porous, remains very challenging. At the moment, the development of integrated screening assays for these proteins is also limited by protein availability. In fact, to produce high enough quantities of solubilized or reconstituted membrane proteins for platform integration is still difficult and a major factor that hinders the development of such assays.

\subsection{References}

1 Rask-Andersen, M.; Almén, M. S.; Schiöth, H. B. Nat. Rev. Drug Discovery 2011, 10, 579590.

2 Tiefenauer, L.; Demarche, S. Materials 2012, 5, 2205-2242.

3 Overington, J. P.; Al-Lazikani, B.; Hopkins, A. L. Nat. Rev. Drug Discovery 2006, 5, 993996.

4 Molecular Cell Biology; 6th ed.; Lodish, H.; Berk, A.; Kaiser, C. A.; Krieger, M.; Scott, M. P.; Bretscher, A.; Ploegh, H.; Matsudaira, P., Eds.; W.H. Freeman \& Co Ltd, 2007.

5 Demarche, S.; Sugihara, K.; Zambelli, T.; Tiefenauer, L.; Voeroes, J. Analyst 2011, 136, 1077-1089.

6 Kresak, S.; Hianik, T.; Naumann, R. L. C. Soft Matter 2009, 5, 4021-4032. 
7 Korman, C. E.; Megens, M.; Ajo-Franklin, C. M.; Horsley, D. A. Langmuir 2013, 29, 44214425.

Kumar, K.; Isa, L.; Egner, A.; Schmidt, R.; Textor, M.; Reimhult, E. Langmuir 2011, 27, 10920-10928.

9 Jonsson, P.; Jonsson, M. P.; Hook, F. Nano Lett. 2010, 10, 1900-1906.

10 Jonsson, P.; Beech, J. P.; Tegenfeldt, J. O.; Hook, F. J. Am. Chem. Soc. 2009, 131, 52945297.

11 Stimberg, V. C.; Bomer, J. G.; van Uitert, I.; van den Berg, A.; Le Gac, S. Small 2013, 9, 1076-1085.

12 Zagnoni, M. Lab Chip 2012, 12, 1026-1039.

13 Hutter, I.; Muller, E.; Kristiansen, P. M.; Kresak, S.; Tiefenauer, L. Microfluid. Nanofluid. 2013, 14, 421-429.

14 Sugihara, K.; Delai, M.; Szendro, I.; Guillaume-Gentil, O.; Voros, J.; Zambelli, T. Sens. Actuators, B 2012, 161, 600-606. Kocun, M.; Janshoff, A. Small 2012, 8, 847-851. Kocun, M.; Lazzara, T. D.; Steinem, C.; Janshoff, A. Langmuir 2011, 27, 7672-7680. Mey, I.; Stephan, M.; Schmitt, E. K.; Muller, M. M.; Ben Amar, M.; Steinem, C.; Janshoff, A. J. Am. Chem. Soc. 2009, 131, 7031-7039. Steltenkamp, S.; Muller, M. M.; Deserno, M.; Hennesthal, C.; Steinem, C.; Janshoff, A. Biophys. J. 2006, 91, 217-226. Tanaka, M.; Sackmann, E. Nature 2005, 437, 656-663.

20 Bally, M.; Bailey, K.; Sugihara, K.; Grieshaber, D.; Voros, J.; Stadler, B. Small 2010, 6, 2481-2497.

21 Kaufmann, M.; Jia, Y.; Werner, C.; Pompe, T. Langmuir 2011, 27, 513-516.

22 El-Khouri, R. J.; Bricarello, D. A.; Watkins, E. B.; Kim, C. Y.; Miller, C. E.; Patten, T. E.; Parikh, A. N.; Kuhl, T. L. Nano Lett. 2011, 11, 2169-2172.

23 Santonicola, M. G.; Memesa, M.; Meszynska, A.; Ma, Y. J.; Vancso, G. J. Soft Matter 2012, 8, 1556-1562. Han, X. J.; Studer, A.; Sehr, H.; Geissbuhler, I.; Di Berardino, M.; Winkler, F. K.; Tiefenauer, L. X. Adv. Mater. 2007, 19, 4466-4470. Reimhult, E.; Kumar, K. Trends Biotechnol. 2008, 26, 82-89. Tiefenauer, L. X.; Studer, A. Biointerphases 2008, 3, FA74-FA79. Reimhult, E.; Kumar, K.; Knoll, W. Nanotechnology 2007, 18, 275303.

28 Schmitt, E. K.; Nurnabi, M.; Bushby, R. J.; Steinem, C. Soft Matter 2008, 4, 250-253.

29 Lazzara, T. D.; Carnarius, C.; Kocun, M.; Janshoff, A.; Steinem, C. ACS Nano 2011, 5, 69356944. 
Lazzara, T. D.; Kliesch, T.-T.; Janshoff, A.; Steinem, C. ACS Appl. Mater. Interfaces 2011, 3, 1068-1076.

Sugihara, K.; Voeroes, J.; Zambelli, T. ACS Nano 2010, 4, 5047-5054.

Jeon, T. J.; Malmstadt, N.; Poulos, J. L.; Schmidt, J. J. Biointerphases 2008, 3, FA96-FA100.

Malmstadt, N.; Jeon, L. J.; Schmidt, J. J. Adv. Mater. 2008, 20, 84-89.

Sugihara, K.; Voros, J.; Zambelli, T. J. Phys. Chem. B 2010, 114, 13982-13987.

Essential Cell Biology; 2nd ed.; Alberts, B.; Bray, D.; Hopkin, K.; Johnson, A.; Lewis, J.; Raff, M.; Roberts, K.; Walter, P., Eds.; Garland Science, 2003.

Studer, A.; Han, X.; Winkler, F. K.; Tiefenauer, L. X. Colloids Surf., B 2009, 73, 325-331.

Wang, H. L.; Chung, T. S.; Tong, Y. W.; Meier, W.; Chen, Z. C.; Hong, M. H.; Jeyaseelan, K.; Armugam, A. Soft Matter 2011, 7, 7274-7280.

Roder, F.; Waichman, S.; Paterok, D.; Schubert, R.; Richter, C.; Liedberg, B.; Piehler, J. Anal. Chem. 2011, 83, 6792-6799.

39 Studer, A.; Demarche, S.; Langenegger, D.; Tiefenauer, L. Biosens. Bioelectron. 2011, 26, 1924-1928.

40 Rigler, P.; Ulrich, W. P.; Vogel, H. Langmuir 2004, 20, 7901-7903.

41 Friedrich, M. G.; Plum, M. A.; Santonicola, M. G.; Kirste, V. U.; Knoll, W.; Ludwig, B.; Naumann, R. L. C. Biophys. J. 2008, 95, 1500-1510.

42 Wei, R.; Gatterdam, V.; Wieneke, R.; Tampe, R.; Rant, U. Nat. Nanotechnol. 2012, 7, 257263. 


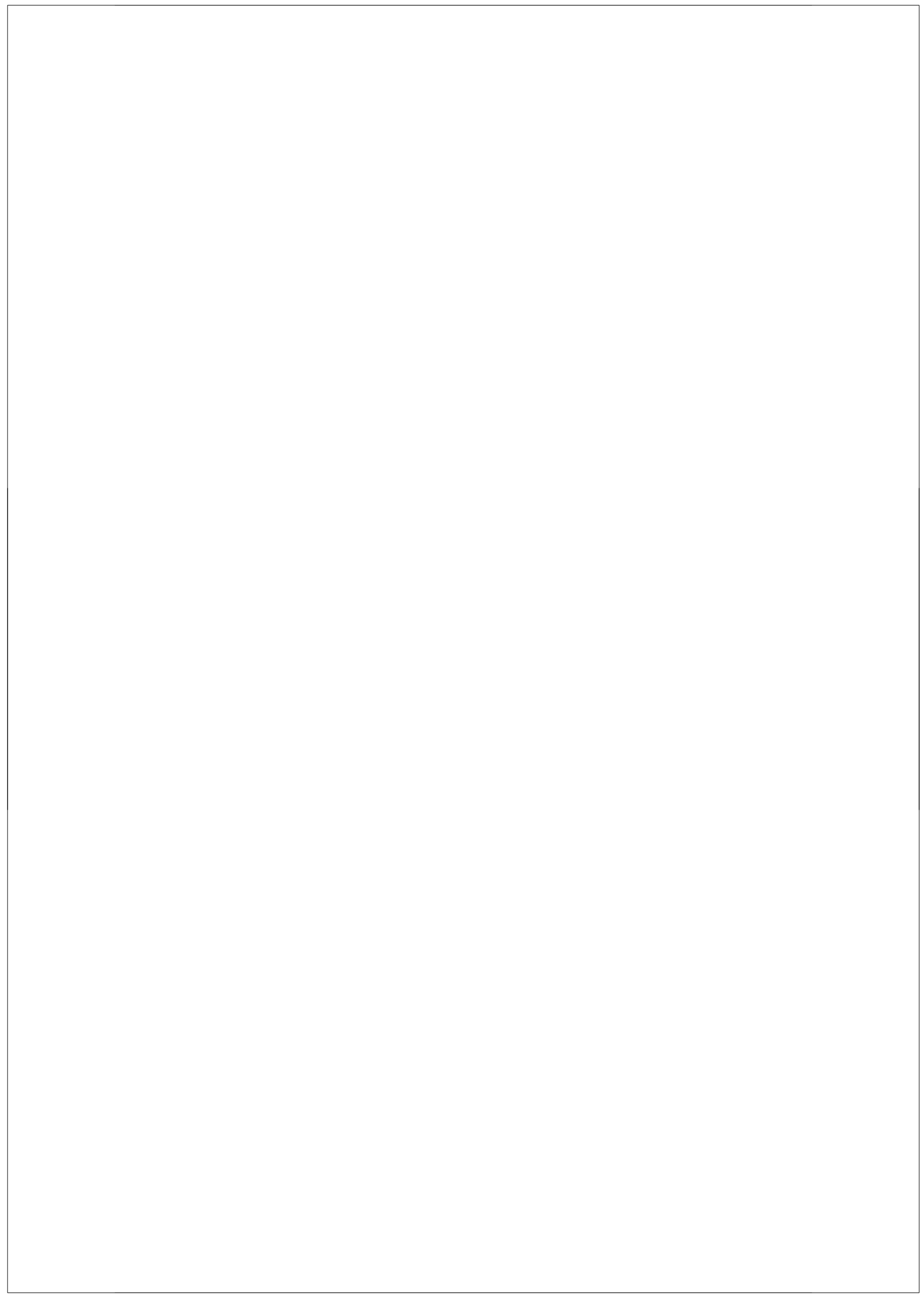




\section{Chapter 4}

Reversible $\mathrm{pH}$-controlled switching of poly(methacrylic acid) grafts for functional biointerfaces*

In this chapter, the description of the experimental work performed for this thesis starts. The grafting of $\mathrm{pH}$-sensitive poly(methacrylic acid) (PMAA) brushes from flat silicon surfaces using surface-initiated atom transfer radical polymerization (SI-ATRP) is presented. The growth kinetics of the PMAA layers was investigated with respect to the composition of the ATRP medium by grafting the polymer in mixtures of water and methanol with different ratios. Adding methanol to the ATRP medium was chosen to improve the surface wettability by the polymerization mixture, in view of the subsequent experiments of grafting PMAA brushes from nanoporous platforms (Chapter 5). The dissociation behavior of the polymer layers was characterized by FTIR titration after incubating the polymer-grafted substrates in phosphate buffer $(P B)$ solutions with varied $\mathrm{pH}$ values. The $\mathrm{pH}$-induced swelling and collapse of the grafted polymer layers were quantified in real time by in-situ ellipsometry in liquid solutions with $\mathrm{pH} 4$ and $\mathrm{pH} 8$. Switching between polymer conformations upon $\mathrm{pH}$ changes is rapid and reversible, and occurs in a range close to physiological conditions, thus opening the opportunity for applications as functional biointerfaces.

\footnotetext{
* This chapter has been published in: Santonicola, M.G.; de Groot, G.W.; Memesa, M.; Meszyńska, A.; Vancso, G.J. Langmuir 2010, 26, 17513-17519
} 


\subsection{Introduction}

Polymer brushes synthesized via surface-initiated polymerizations ("grafting from" approach) are a powerful tool to modify and control the surface properties of materials. In particular, polymer brushes grown using controlled radical polymerization techniques, most notably atom transfer radical polymerization (ATRP), provide robust and reproducible platforms for surface modification, by controlling functionality, density and thickness of the polymer brushes. ${ }^{1,2}$ These platforms are especially relevant for biologically-oriented applications, such as protein assays and biosensor devices. Here the need for reliable functionalization strategies is crucial, since mechanical stability of bioassays over multiple sensing techniques and for several days is often a limiting factor. ${ }^{3}$ In particular, development of screening assays for membrane proteins is of interest, as more than $60 \%$ of therapeutic drugs in use today target these proteins. ${ }^{4}$ Polymer-grafted substrates with tunable surface energy can also be helpful to guide liposome adsorption and formation of lipid membranes with embedded transmembrane proteins. Moreover, interfaces with reversible swollen/collapsed conformations could be useful in micro-porous platforms with controlled ion permeation to selectively probe the functionality of supported membrane protein ion channels. ${ }^{5,6}$ The possibility of reversibly binding macromolecules to surfaces is relevant to a variety of other applications as well, including protein purification by affinity chromatography, where responsive interfaces are exploited for protein immobilization and release under application of external stimuli. ${ }^{7}$

Surfaces with reversible switching between conformations can be achieved by immobilization of dense assemblies of flexible, long-chain polymers (that is polymer brush) containing segments that respond to external stimuli, such as variations of temperature, $\mathrm{pH}$, ionic strength or electric field. ${ }^{8,9}$ Polymer brushes are of great interest due to the specific properties that arise from their surface-confined architecture. The dissociation behavior of $\mathrm{pH}$-responsive polymer brushes bearing carboxylic groups, for example, is largely affected by surface confinement, and the local distribution of charges within the polymer layer is different from that of the bulk solution. ${ }^{10,11}$ As a result, these polymers in the brush configuration exhibit $\mathrm{p} K_{\mathrm{a}}$ values $\left(\mathrm{p} K_{\mathrm{a}}{ }^{\text {bulk }}\right)$ that are shifted relative to the $\mathrm{p} K_{\mathrm{a}}$ value of the polymer surface that is fully accessible by solvent. Recently, Dong and co-workers provided the first experimental evidence of the variation of the degree of dissociation within weak polyelectrolyte brushes on gold surfaces. ${ }^{12}$ Using FTIR titration, the authors determined the effective $\mathrm{p} K_{\mathrm{a}}^{\text {bulk }}$ of PMAA brushes synthesized on 
gold to be in the range 6.9-7.0, which is more than 2 units larger than the value measured at the brush surface $\left(\mathrm{p} K_{\mathrm{a}}^{\text {surf }}\right.$ ) by contact angle titration.

Several procedures are available for the synthesis of polymer brushes on surfaces. ${ }^{13}$ Among these, surface-initiated atom-transfer radical polymerization, ${ }^{14}$ from initiator monolayers immobilized on surface, is especially attractive for its tolerance to water and its compatibility with a wide range of functionalized monomers, as well as for the option of carrying out polymerizations at ambient temperature. ${ }^{15,16}$ ATRP polymerizations in mixtures of water and an alcohol can be used as a compromise between fast polymerization kinetics and the need to achieve polymerization in confined spaces such as micro-sized pores. ${ }^{17}$ However, control of the polymer grafting conditions and how this relates to the responsive behavior of the brushes are poorly understood. In particular, knowledge of the relation between grafting conditions and $\mathrm{pH}$-controlled response of weak polyelectrolyte brushes would greatly improve the design of functional biointerfaces with tunable interfacial energy.

Atomic force microscopy (AFM) is a powerful tool for the characterization of surfacegrafted polymer brushes. ${ }^{18}$ Previously, direct visualization of the swelling and collapse of $\mathrm{pH}$-responsive polymer layers, as well as the brush mechanical performance under compression, were assessed using AFM in liquid environment. ${ }^{19,20}$ However, studies of the reversibility of polymer switching between conformations in real time are difficult to accomplish by AFM due to the long equilibration times required for imaging and the presence of electrostatic interaction between tip and charged polymer surface. Ellipsometry, using polarized light with an angle to the surface, is a rapid and noninvasive optical technique commonly used to characterize thin films with multi-layer structures. Previously, in- situ multiple-angle null ellipsometry was used to examine the swelling behavior of weak poly acid brushes as a function of solution $\mathrm{pH}$ and salt concentration and of mixed polyelectrolyte brushes. ${ }^{21,22}$

Here we investigate the $\mathrm{pH}$-induced swelling and collapsing behavior of poly(methacrylic acid) (PMAA) grafted from silicon surfaces using atom-transfer radical polymerizations in relation to the methanol content of the aqueous ATRP medium. Polymer grafting kinetics and the real-time response of these brushes to variations of the surrounding solution $\mathrm{pH}$ are investigated by in-situ ellipsometry. Reversibility of the $\mathrm{pH}$-induced polymer switching is analyzed in FTIR titration and ellipsometry measurements. With this work we aim at investigating the responsive performance of 
PMAA brush structures for potential integration into platforms that can be used for $\mathrm{pH}$ controlled molecular screening of biological molecules.

\subsection{Results and Discussion}

\subsubsection{PMAA brush growth and characterization from silicon surfaces}

Surface-initiated ATRP was used to graft responsive poly(methacrylic acid) layers from silicon surfaces with immobilized (3-(2-bromoisobutyryl)propyl)dimethylchlorosilane molecules. Initiator layers obtained from deposition in vapor phase were consistently denser than those obtained with immersion in toluene solutions. The technique of contact angle measurements was used to probe the surface energetic state of immobilized initiator and grafted polymer films and could provide valuable information directly related to changes in surface properties as a result of a change in the surrounding medium. The static contact angle of water on the vapor-phase immobilized monolayers was typically $85 \pm 3^{\circ}$ and was found to be independent of the interval of time over which the monolayers were formed for times from $1 \mathrm{~h}$ to $24 \mathrm{~h}$. For initiator monolayers assembled in liquid phase (toluene solution), water contact angles were in the range $40^{\circ}-45^{\circ}$ after $18 \mathrm{~h}$ immersion.

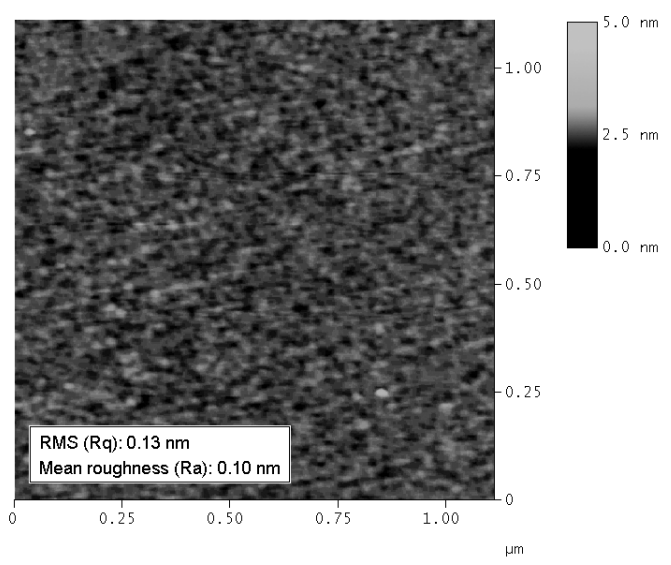

Figure 4.1: High resolution topography image by TM-AFM of ATRP initiator layer immobilized on silicon surface via vapor-phase deposition for $12 \mathrm{~h}$.

The surface morphology of the initiator monolayers in air was evaluated by AFM in tapping mode. After a vapor-phase deposition of $12 \mathrm{~h}$, monolayers have a thickness of $1.2 \pm 0.1 \mathrm{~nm}$ (step-height method) and an average surface roughness (root mean square, $\mathrm{rms}$ ) of $0.13 \mathrm{~nm}$ (scan size $1 \times 1 \mu^{2}$ ) (Figure 4.1). After polymerization, the water 
contact angle shows a clear decrease from $82 \pm 2^{\circ}$ for the initiator-covered silicon to 10 $\pm 3^{\circ}$, indicating the presence of a highly hydrophilic film immobilized on the surface.

The initiator-covered silicon surfaces were investigated by XPS to assess the quality of the deposited monolayers (Figure 4.2). The high resolution scan of the $C$ 1s peak was fitted for the different carbon species present in the initiator molecule, and their relative percentage was found to be in agreement with the carbon content of the molecule (Figure $4.2 \mathrm{~b}$ ). In the spectrum of the clean silicon wafer, no significant $\mathrm{C}$ 1s peak was detected (Figure 4.2a).
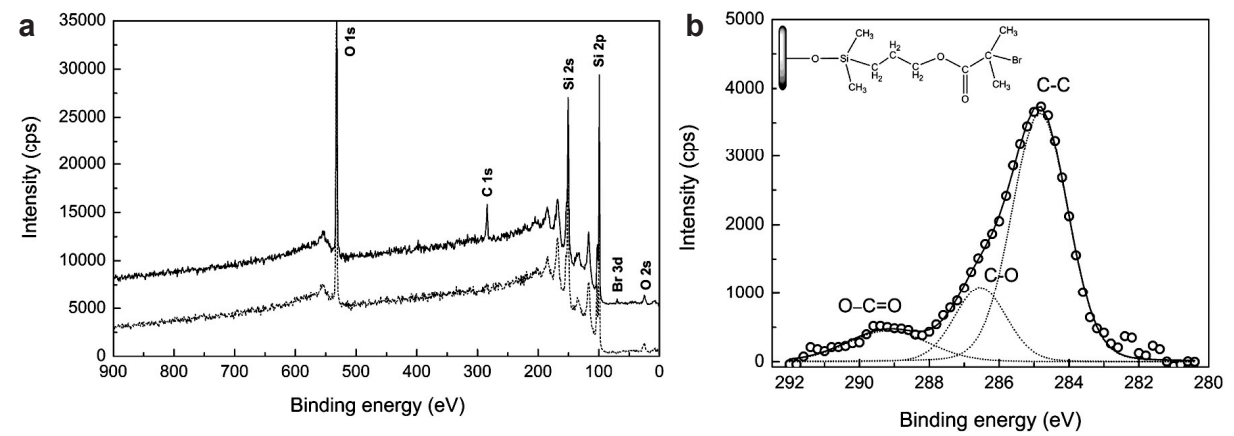

Figure 4.2: (a) XPS survey spectra (1.5 keV) of the ATRP initiator monolayer (solid line) and bare silicon surface (dashed line). (b) High-resolution XPS spectrum with carbon 1s band (0) and peak fitting (lines) of the surface-immobilized ATRP initiator (3-(2bromoisobutyryl)propyl)dimethylchlorosilane. The fitted relative percentages of the carbon species are $68.7 \%, 19.0 \%$ and $12.4 \%$ for $C-C, C-O$, and $\mathrm{O}-\mathrm{C}=\mathrm{O}$, respectively.

After polymerization, the near-surface composition of a 33-nm thick PMAA layer was determined using XPS. The carbon:oxygen molar composition was 64.9:35.1, which is comparable to the theoretical value of $66.7: 33.3$ for PMAA. The high resolution $C$ is spectrum was analyzed to determine the relative content of the carbon species in the polymer layer. The spectrum could be fitted with two components: a peak at $288.5 \mathrm{eV}$ corresponding to the sp2-hybridized carbon in the carboxyl group and a peak at 284.8 $\mathrm{eV}$ attributed to the sp3-hybridized carbon of the alkyl chain (Figure 4.3). A molar composition of 76.1:23.9 was determined from the integrated area of the two fitted components, in good agreement with the value of 75.0:25.0 expected for PMAA. In addition, XPS analysis of PMAA brushes that were treated after polymerization using different procedures was useful to assess the presence of $\mathrm{Cu}^{2+}$ ions and bipyridine 
molecules trapped inside the films, and showed that these residues could be effectively removed by treatment with $0.1 \mathrm{M}$ EDTA solution.

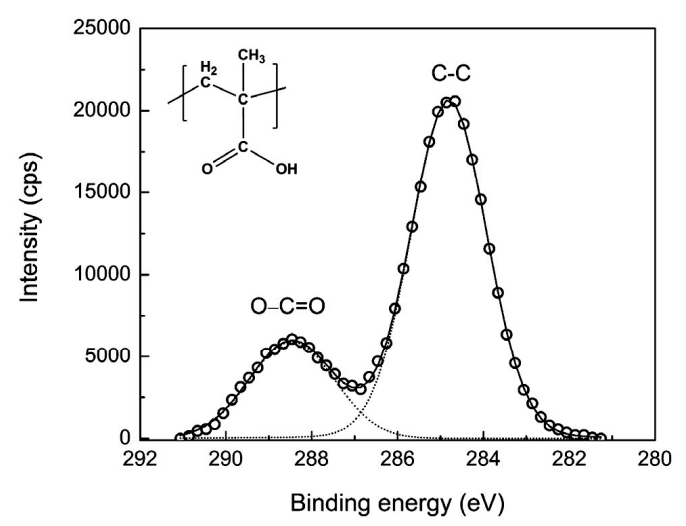

Figure 4.3: High-resolution XPS spectrum with carbon 1s band (o) and peak fitting (lines) of PMAA brushes grafted from silicon surfaces (SI-ATRP in water medium).

The evolution of the ellipsometric brush thickness with polymerization time with respect to the water/methanol content of the ATRP medium was investigated. Figure 4.4 shows the dry thickness of PMAA brushes grafted using ATRP in water and water/methanol 50:50 v/v medium after different polymerization times. Polymer brushes exhibit fast growth kinetics when synthesized using ATRP in both media. No significant difference can be observed in the PMAA thickness after $1 \mathrm{~h}$ in both cases. PMAA of low thicknesses ( $30 \mathrm{~nm}$ ) were synthesized using water as ATRP medium, suggesting early termination of the polymerization in water. In contrast, in the water/methanol mixture with ratio 50:50 v/v, PMAA grows to a thickness of $\sim 90 \mathrm{~nm}$. Polymer layers grafted in water are homogenous with a standard deviation of the dry thickness across the surface of less than $2 \mathrm{~nm}$. 


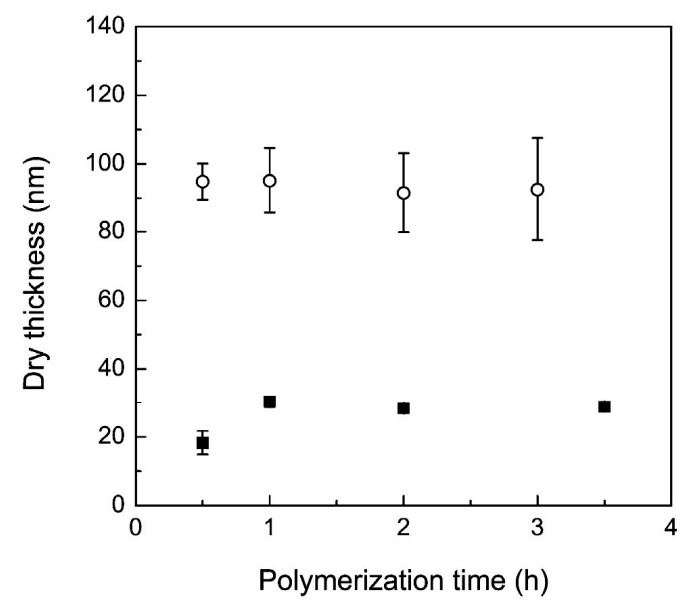

Figure 4.4: Ellipsometric thicknesses of dry PMAA layers on silicon surface as a function of the polymerization time in different SI-ATRP grafting medium: water ( $\mathbf{})$ and water/methanol 50:50 $\mathrm{v} / \mathrm{v}(\mathrm{O})$. Error bars are given by the standard deviation of an array of 25 measurements across the polymer brush surface (sampled area $5 \times 5 \mathrm{~mm}^{2}$ ).

\subsubsection{Characterization of $\mathrm{pH}$-sensitive behavior of PMAA brushes}

Prior to examining the $\mathrm{pH}$-controlled switching of the PMAA brushes in solutions with different $\mathrm{pH}$ values, the stability of the initiator monolayers in such solutions was investigated. In acidic solutions cleavage of the initiator molecules from silicon surfaces can occur, leading to detachment of the grafted polymer layer. For this study we measured variations of the wetting properties of the initiator monolayers after incubation of the functionalized silicon surfaces in PB solutions with different $\mathrm{pH}$ values. Table 4.1 shows variations of the static water contact angle on initiator monolayers as a function of the incubation time. Each of the substrates was incubated sequentially for 0 , 15, 60 and $120 \mathrm{~min}$. In solutions with a pH value of 3, the water contact angle of the initiator monolayer shows a clear decrease of about $12^{\circ}$ after $2 \mathrm{~h}$ of immersion, consistent with cleavage of the initiator molecules from the silicon surface. For all other $\mathrm{pH}$ values, the difference of the contact angle after $120 \mathrm{~min}$ of immersion is within $5^{\circ}$. In view of these results, the swelling and collapsing properties of silicon-grafted PMAA brushes were investigated in $\mathrm{PB}$ solutions with $\mathrm{pH}$ values between 4 and 8 , where the brushes exhibit good stability. 
Table 4.1: Changes in the wetting properties of (3-(2bromoisobutyryl)propyl)dimethylchlorosilane monolayers immobilized on silicon surfaces during incubation in PB solutions ( $50 \mathrm{mM}$ phosphate) with different $\mathrm{pH}$ values. Each contact angle value is a mean of five independent measurements. Standard deviation is less than $3 \%$.

\begin{tabular}{ccccc}
\hline \multicolumn{5}{c}{ Static water contact angle [ ${ }^{\circ}$ ] } \\
& \multicolumn{5}{c}{$\mathrm{pH}$} & $0 \mathrm{~min}$ & $15 \mathrm{~min}$ & $60 \mathrm{~min}$ & $120 \mathrm{~min}$ \\
\hline 3.0 & 82 & 76 & 74 & 70 \\
4.0 & 83 & 81 & 79 & 78 \\
5.0 & 83 & 80 & 79 & 77 \\
6.0 & 85 & 84 & 82 & 80 \\
7.0 & 85 & 84 & 82 & 80 \\
8.0 & 83 & 81 & 80 & 79 \\
\hline
\end{tabular}

The dissociation behavior of the carboxylic acid groups of silicon-grafted PMAA layers was evaluated by FTIR spectroscopy. Titration experiments were conducted by recording FTIR spectra after incubating the polymer-grafted substrates in PB solutions with different $\mathrm{pH}$ values. Figure 4.5 shows the FTIR absorption spectra for PMAA brushes treated with different buffer solutions. The characteristic band peaks of protonated and deprotonated carboxylic acid groups appear at $1705 \mathrm{~cm}^{-1}$ ( $\mathrm{C}=\mathrm{O}$ stretching of $\mathrm{COOH}$ ) and at $1558 \mathrm{~cm}^{-1}$ (asymmetric stretching band of $\mathrm{COO}^{-}$) respectively. The integrated areas under these characteristic bands were used to determine the degree of dissociation of the carboxylic acid groups as a function of the solution $\mathrm{pH}$, following previously reported procedures. $^{23,24}$ Experimentally determined titration curves were fitted to a sigmoidal function to determine the $\mathrm{pH}$ at which the degree of dissociation of the carboxylic acid groups is 0.5 , that is the effective $\mathrm{p} K_{\mathrm{a}}$ of the polymer in the brush configuration (Figure 4.6). The effective $\mathrm{p} K_{\mathrm{a}}^{\text {bulk }}$ of PMAA grafted from silicon surface is $6.5 \pm 0.1$. This value was found to be independent of PMAA grafting conditions, in terms of polymerization time and water/methanol ratio of the ATRP medium, when similar silicon-immobilized SAMs of initiator molecules are used. pH-responsive polymers grafted from surfaces using ATRP exhibit an effective $\mathrm{p} K_{\mathrm{a}}$ that is larger than the $\mathrm{p} K_{\mathrm{a}}$ of the monomer in 
solution. The larger $\mathrm{p} K_{\mathrm{a}}$ values are due to the unique properties of polymer brushes, namely the high grafting density and polymer chain segment density, which lead to a strong ion confinement effects.

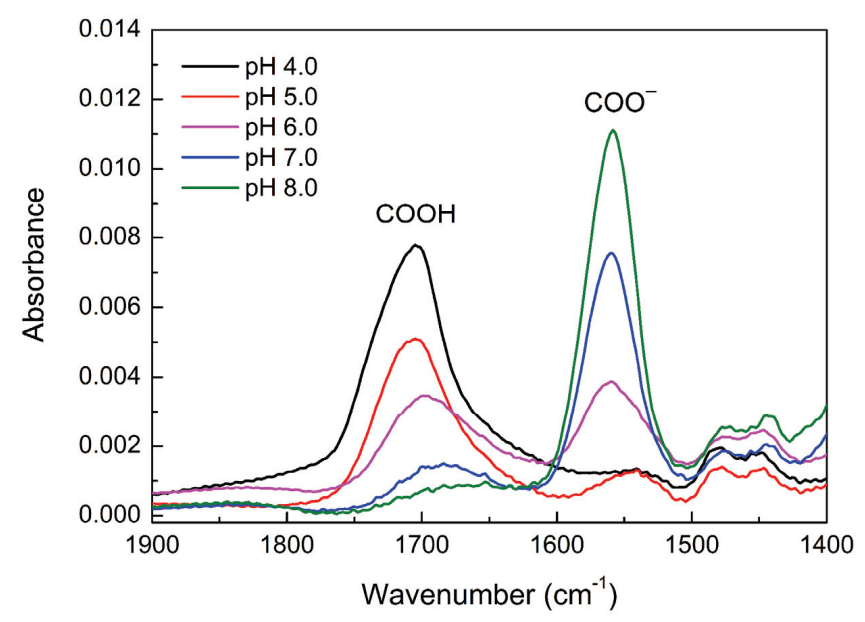

Figure 4.5: Carbonyl absorption region in FTIR spectra of PMAA brushes grafted from silicon surfaces (ATRP in water medium) after incubation in PB solutions (50 mM phosphate) with different $\mathrm{pH}$ values.

As a result, acid groups in the uppermost layers of the brush are easier to ionize than the acid groups buried down to the substrate surface. Theoretical simulations on weak polyelectrolyte layers showed that there is a strong coupling between the bulk salt concentration, the solution $\mathrm{pH}$, and the polymer density, which results in local degree of charging within the polymer brush, leading to the prediction that $\mathrm{p} K_{\mathrm{a}}^{\text {bulk }}$ is larger than $\mathrm{p} K_{\mathrm{a}}^{\text {surf }}{ }^{25}$ This behavior was experimentally proved for brushes of PAA and PMAA grown on gold surfaces using ATRP, where it was shown that the effective $\mathrm{p} K_{\mathrm{a}}^{\text {bulk }}$ values exceed the effective $\mathrm{p} K_{\mathrm{a}}^{\text {surf }}$ values by $\sim 2$ units. ${ }^{12}$ Here, for polymer brushes grafted from silicon surfaces the smaller difference observed between $\mathrm{p} K_{\mathrm{a}}^{\text {bulk }}$ and $\mathrm{p} K_{\mathrm{a}}^{\text {surf }}$ can be explained considering the different grafting density of the initiator molecules that are used in the synthesis. Polymer brushes grafted from gold surface can achieve higher grafting density due to the smaller cross-sectional area of the thiol-ended initiator molecules, and therefore exhibit a larger shift of the $\mathrm{p} K_{\mathrm{a}}{ }^{\text {bulk }}$ values. 


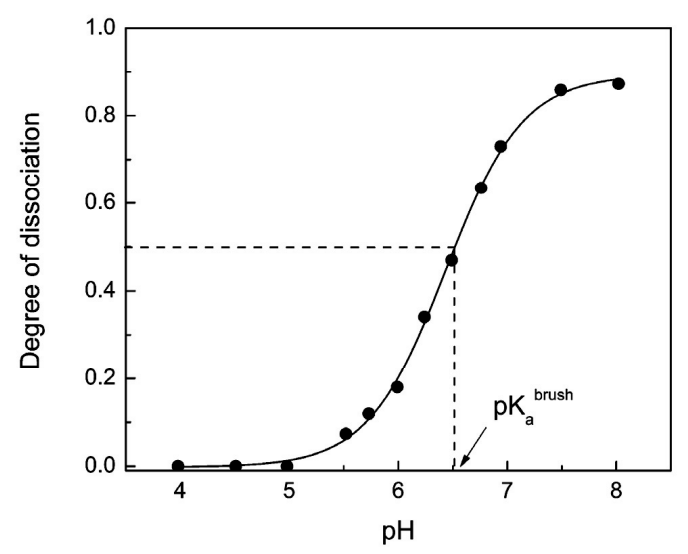

Figure 4.6: Degree of dissociation of carboxylic acid groups of a PMAA brush grafted from silicon surface (ATRP in water/methanol 50:50 mixture) as a function of the PB solution $\mathrm{pH}$. The solid line is a fit to a sigmoidal function.

The reversibility of the switching behavior of PMAA brushes grafted in ATRP aqueous solvent was investigated by FTIR and in situ ellipsometry using a liquid cell. Figure 4.7a and $4.7 \mathrm{~b}$ show the degree of dissociation of carboxylic groups and the thickness of the polymer layer, respectively, when switching the $\mathrm{pH}$ of the PB medium between 4 and 8 . The degree of dissociation varies reversibly with the $\mathrm{pH}$ change of the incubation medium. In acidic medium ( $\mathrm{pH} 4$ ) carboxylic acid groups are fully protonated, whereas at $\mathrm{pH} 8$ the degree of dissociation approaches the value of 1 . At the same time, at pH 4 the neutral polymer layers are in a collapsed state, and they swell due to the repulsion between the carboxylate groups at $\mathrm{pH}$ 8. PMAA layers synthesized via ATRP in water medium with dry thickness of $28 \mathrm{~nm}$ swell to a value of $\sim 66 \mathrm{~nm}$ in PB medium with pH 4 . This layer swells to a height of $104 \mathrm{~nm}$ in PB with $\mathrm{pH}$ 8. We define the brush swelling factor as the ratio of the maximum and minimum height values corresponding to the extended and collapsed state of the polymer brush at pH 8 and 4 . Here the swelling factor is $1.57 \pm 0.12$ (average of 4 cycles). Furthermore, at the end of each swelling/collapsing cycle the dry thickness of the polymer brush was determined again to evaluate any polymer loss from the substrates. Measurements showed no significant difference in the values of the ellipsometric angles before and after swelling/collapsing cycles within the inhomogeneity of the polymer film surface, thus supporting the argument that SI-ATRP can be used to synthesize very robust polymer brush structures on surfaces. The time of the polymer brush response with $\mathrm{pH}$ change was also 
investigated by ellipsometry kinetics. The polymer brush reached the equilibrium swollen/collapsed conformation within $1 \mathrm{~min}$ and was stable in the following $30 \mathrm{~min}$.

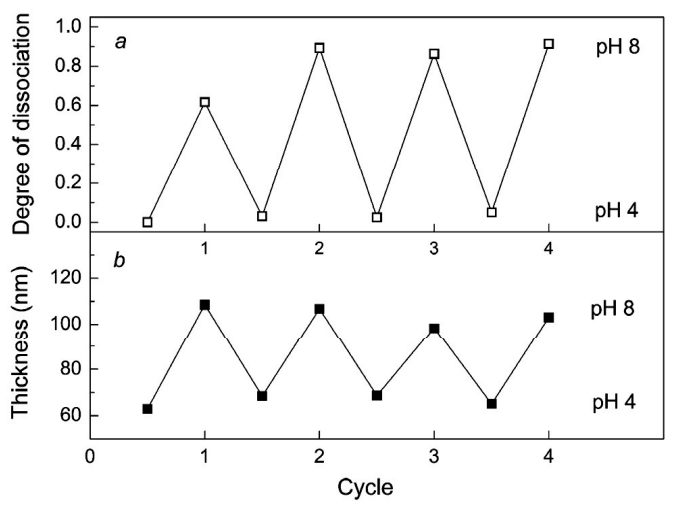

Figure 4.7: Reversibility of the pH-induced switching of a PMAA brush (dry thickness $28 \mathrm{~nm}$ ) grafted from silicon surface as investigated by FTIR (a) and in situ ellipsometry in PB medium (b).

The swelling behavior of the PMAA layers in PB solutions was evaluated by in situ AFM in liquid environment. Samples were scratched with plastic tweezers to remove mechanically part of the polymer layer and sonicated gently in ethanol for 1-2 min to remove the detached polymer. Next, they were immersed in PB solution and let to equilibrate for at least $1 \mathrm{~h}$ before images were taken. The height of the polymer brush was determined by AFM operated in contact mode with minimal loading force as the difference between the scratched region and the surrounding intact polymer layer. PMAA brushes with dry thickness of $31 \mathrm{~nm}$ (Figure 4.8a) show a swollen step-height profile of $115 \mathrm{~nm}$ when immersed in a PB solution with $\mathrm{pH} 8$ (Figure 4.8b). Both values are in agreement with those determined by ellipsometry. In liquid environment the thickness of the swollen polymer brush as determined by ellipsometry is $10 \%$ smaller than the height determined using AFM, which is expected when an isotropic layer model is used for the fitting of ellipsometric angles. In addition, swollen polymer layers can be subject to compression during AFM imaging in contact mode due to the load applied by the tip. Previously, for PMAA brushes synthesized on gold surfaces using SI-ATRP height compressions of approximately 30\% were reported under applied loads of $26 \mathrm{nN}$. In our measurements load forces of $10 \mathrm{nN}$ at most and relatively fast scan speeds (10 $\mu \mathrm{m} / \mathrm{s})$ were applied during imaging in order to reduce polymer brush compression to a minimum. 

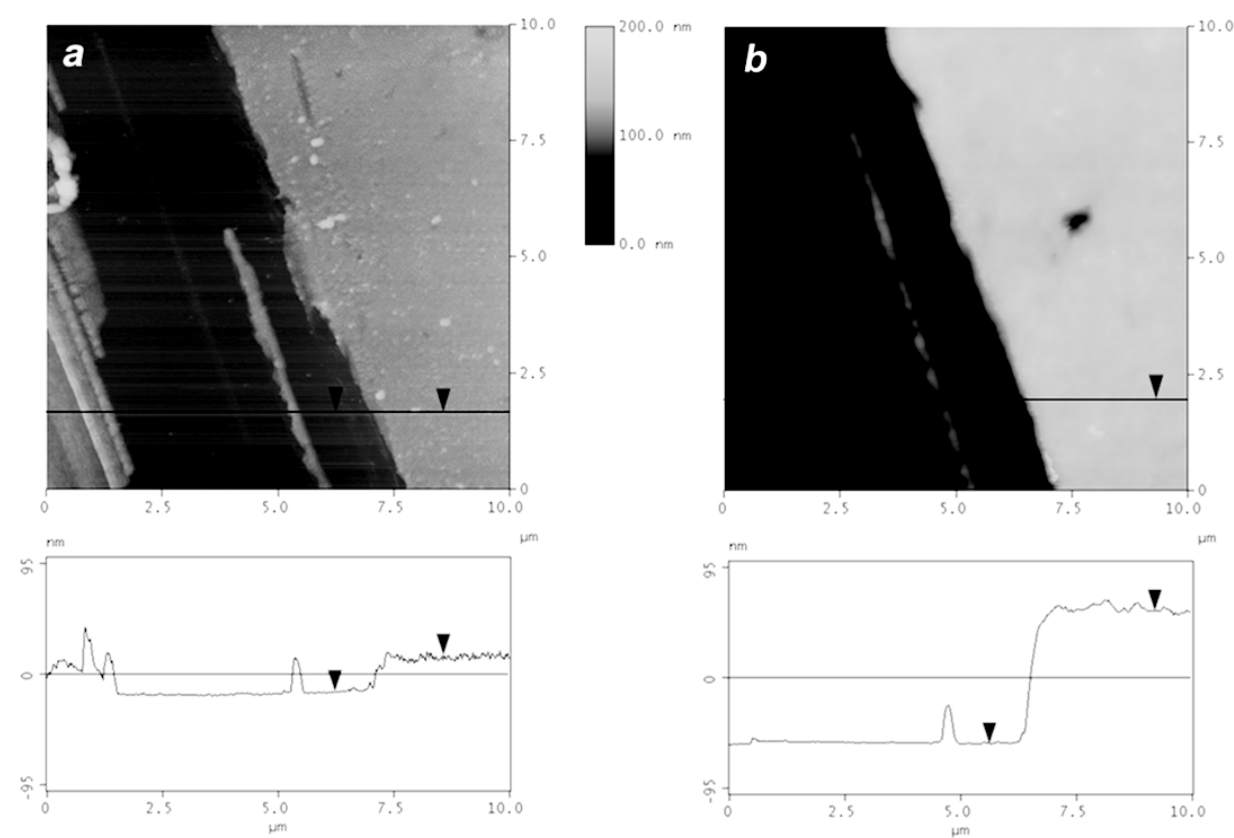

Figure 4.8: Topography image by CM-AFM of PMAA brush synthesized using SI-ATRP in water medium (polymerization time $1 \mathrm{~h}$ ) in air (a) and in PB solution of $\mathrm{pH} 8$ (b). Histograms show the evaluation of the average height of the polymer layer from scratched and unscratched regions: measured step-heights are $31 \mathrm{~nm}$ and $115 \mathrm{~nm}$ for dry environment and for PB with $\mathrm{pH}$ 8, respectively.

The effect of the polymerization medium, water or water/methanol, on the swelling properties of PMAA-grafted layers was investigated. PMAA brushes of similar thickness of $~ 90 \mathrm{~nm}$ were chosen for comparison, and the $\mathrm{pH}$-induced swelling and collapsing was evaluated in situ in aqueous environment using a liquid ellipsometric cell. The thickness and the refractive index of the polymer layers were obtained from the fitting procedure. Figure 4.9 shows the evolution of the polymer film thickness and refractive index while changing the solution $\mathrm{pH}$ between the values of 4 and 8 . For brushes polymerized in water, the swollen thickness varies between $\sim 182 \mathrm{~nm}$ and $\sim 230 \mathrm{~nm}$ at $\mathrm{pH} 4$ and $\mathrm{pH} 8$, respectively, corresponding to a swelling factor of $1.26 \pm 0.04$ (Figure 4.9a). On the other hand, PMAA brushes polymerized in water/methanol 50:50 v/v mixtures with same dry thickness swell to a thickness of $\sim 207 \mathrm{~nm}$ in PB solution with $\mathrm{pH} 4$ and extend to $\sim 229$ $\mathrm{nm}$ at $\mathrm{pH}$ 8. In this case the swelling factor is $1.11 \pm 0.03$, which is lower than for brushes polymerized in water. This behavior is not fully understood and could be related to a different polymer brush density achieved by polymerizing PMAA in a water/methanol 
mixture with respect to an aqueous medium as a consequence of different wetting of the surface-immobilized ATRP initiator monolayer (contact angle $\sim 85^{\circ}$ ). Experiments were conducted to investigate differences in the grafting density of the polymer brushes grown at the conditions above. However, due to the small size of the grafted substrates, experimental determination of PMAA grafting density by polymer layer cleavage and subsequent molecular weight assessment by GPC was not feasible. At the same time, polymerization in solution in the presence of a sacrificial initiator gave inconsistent results, due to the need to use a water-soluble initiator (2-bromo-isobutyric acid), in combination with the same polymerization medium used in our surface-initiated ATRP, which resulted in an uncontrolled polymerization. In fact, grafting density determination with the use of sacrificial initiators is limited to those cases where the growth of free polymers in solution and grafted polymer layers exhibit similar kinetics, as it was shown for SI-ATRP in non-aqueous solvents. ${ }^{26}$ It appears clear, however, that the polymer brushes synthesized in this work are in the high grafting density regime because they exhibit large values of $\mathrm{p} K_{\mathrm{a}}{ }^{\text {bulk }}$, as determined by FTIR titration, which is consistent with the theory of charge regulation within weak polyelectrolytes grafted layers of high density. ${ }^{10,11}$
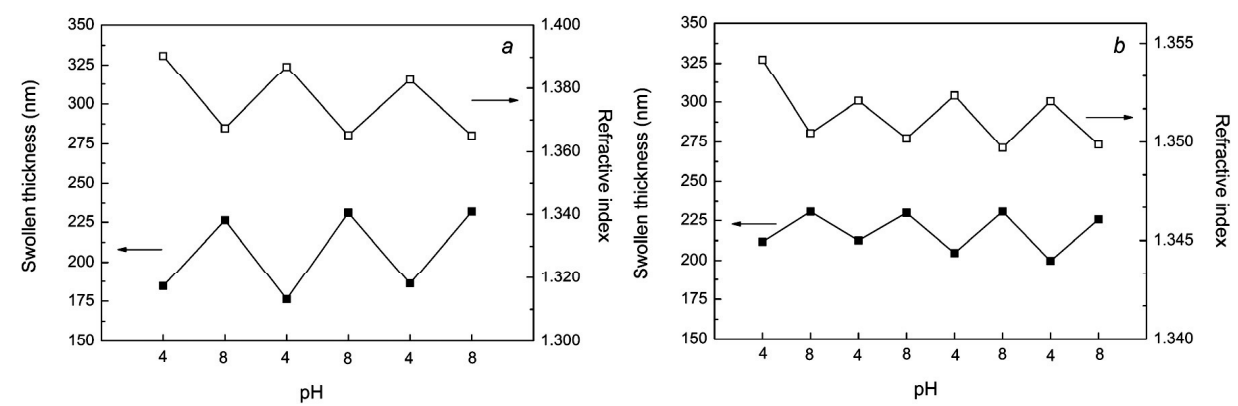

Figure 4.9: Swelling and collapsing behavior of PMAA brush (dry thickness $\sim 90 \mathrm{~nm}$ ) on silicon substrate by in situ ellipsometry in response to changes of $\mathrm{pH}$ in PB medium ( $50 \mathrm{mM}$ phosphate). Polymer layers grafted using SI-ATRP in water medium (a) and water/methanol mixture of ratio 50:50 v/v (b). 


\subsection{Conclusions}

Polymer brushes synthesized by surface-initiated atom-transfer radical polymerization provide a robust and reproducible platform for surface modification. In particular, $\mathrm{pH}$ responsive polymers offer the possibility of achieving rapid and reversible switching between conformations, which can be induced locally, making them ideal candidates for miniaturized devices with fast response times. Here we show that $\mathrm{pH}$-responsive PMAA layers grafted from initiator-immobilized silicon surfaces using ATRP exhibit reversible and rapid switching between conformations, and can withstand several cycles of swelling and collapsing with no significant loss from the surfaces. Silicon-grafted PMAA films with thicknesses from 20 to $100 \mathrm{~nm}$ show consistent and reproducible switching between extended and collapsed states at pH 8 and 4, respectively, as determined by in situ ellipsometry, with swelling factors that can be tuned by varying the water/methanol ratio in the ATRP medium in addition to the polymerization time. For all conditions investigated here, FTIR titration experiments show that the effective $\mathrm{p} K_{\mathrm{a}}$ of the PMAA layers is shifted to larger values than the monomer $\mathrm{p} K_{\mathrm{a}}$ due to ion confinement effects in the dense brush structures. This shifting of the $\mathrm{p} K_{\mathrm{a}}$ in the brush configuration is especially relevant for biologically-oriented applications of these polymers, such as functionalization of platforms for protein assays or biosensing, as switching between conformations can be triggered in a $\mathrm{pH}$ range closer to physiological conditions where protein functionality is optimal.

\subsection{Experimental Section}

Materials: Allyl 2-bromo-2-methylpropionate (CAS no. 40630-82-8, 98\%), chlorodimethylhydrosilane (CAS no. 1066-35-9, 98\%), chloroplatinic acid hexahydrate (CAS no. 18497-13-7, $\geq 37.50 \%$ Pt basis), sodium methacrylate (CAS no. 5536-61-8, 99\%), CuBr (CAS no. 7787-70-4, 99.999\%), $\mathrm{CuBr}_{2}$ (CAS no. 7789-45-9, 99.999\%), 2,2'-bipyridine (CAS no. 366-18-7, $\geq 99.0 \%$ ) were purchased from Sigma-Aldrich and used without further purification. All solvents were of high purity, and deionized water from a Milli-Q purification system (Millipore Advantage A10) was used throughout. PB solutions (50 $\mathrm{mM}$ phosphate) with various $\mathrm{pH}$ values were prepared by titrating aliquots from the same stock ( $\mathrm{pH} 7.4$ ) using $0.1 \mathrm{~N} \mathrm{HCl}$ or $\mathrm{KOH}$ solutions.

Surface-initiated polymerization of PMAA: Polymer layers were grafted from silicon surfaces with immobilized (3-(2-bromoisobutyryl)propyl)dimethylchlorosilane molecules. Synthesis of the ATRP initiator was conducted as previously reported. ${ }^{27}$ Allylic 
ester $(0.6 \mathrm{ml})$ was mixed with $4 \mathrm{ml}$ of dimethylchlorosilane. Few granules of chloroplatinic acid ( $\geq 37.5 \% \mathrm{Pt}$ ) were added and the mixture was stirred under nitrogen and in the dark for $48 \mathrm{~h}$ (room temperature). At the end of the reaction the excess chlorosilane was removed under reduced pressure. The quality of the silane initiator was determined using standard ${ }^{1} \mathrm{H}$ NMR analysis $\left[\mathrm{CDCl}_{3}, 300 \mathrm{MHz} ; \delta=0.43\left(\mathrm{Si}\left(\mathrm{CH}_{3}\right)_{2}, \mathrm{~s}, 6 \mathrm{H}\right)\right.$; $0.87\left(\mathrm{SiCH}_{2}, \mathrm{~m}, 2 \mathrm{H}\right) ; 1.76-1.81\left(\mathrm{CH}_{2}, \mathrm{~m}, 2 \mathrm{H}\right) ; 1.93\left(\mathrm{C}\left(\mathrm{CH}_{3}\right)_{2}, \mathrm{~s}, 6 \mathrm{H}\right) ; 4.14-4.19\left(\mathrm{CH}_{2} \mathrm{O}, \mathrm{m}\right.$, $2 \mathrm{H})$ ]. Initiator molecules were immobilized on freshly cleaned silicon surfaces by vapor phase deposition in a desiccator under vacuum. Prior to the deposition, silicon slides $\left(10 \times 10 \mathrm{~mm}^{2}\right.$ or $\left.15 \times 15 \mathrm{~mm}^{2}\right)$ were sonicated in ethanol and in deionized water for 15 min. Subsequently, the slides were cleaned in piranha solution $\left(\mathrm{H}_{2} \mathrm{SO}_{4} / \mathrm{H}_{2} \mathrm{O}_{2}\right.$ 70:30 v/v) for 30 min (warning: piranha solution reacts strongly with organic compounds and should be handled with extreme caution), rinsed extensively with water, ethanol, and dried in a stream of nitrogen. The initiator deposition time was kept constant (generally $16 \mathrm{~h})$, after which the substrates were extracted with dichloromethane, rinsed with ethanol and dried under nitrogen gas. Initiator-immobilized substrates were immediately used for surface-initiated ATRP.

The polymerization was carried out in water/methanol mixtures of different ratios at room temperature. The initiator-covered silicon substrates were placed in dry vials and purged with argon for $1 \mathrm{~h}$. CuBr (143.5 mg, $1 \mathrm{mmol}), \mathrm{CuBr}_{2}(22.3 \mathrm{mg}, 0.1 \mathrm{mmol})$, and 2,2'-bipyridine (343.6 mg, $2.2 \mathrm{mmol}$ ) were added to a $25 \mathrm{~mL}$ Schlenk flask equipped with a magnetic stir bar. Air in this flask was evacuated and replaced with argon three times. Sodium methacrylate $(5.40 \mathrm{~g}, 50 \mathrm{mmol})$ was dissolved in the ATRP medium $(10 \mathrm{~mL})$. The monomer solution was degassed with argon for $30 \mathrm{~min}$ and then transferred into the polymerization Schlenk flask using a syringe previously purged in argon atmosphere. The mixture was stirred under argon for about 10 min until a viscous brown solution was obtained. Next, the polymerization solution was injected into each reaction vial, adding enough solution to submerge each sample completely. After polymerization, samples were removed from the vials, washed with deionized water, gently sonicated in EDTA solution (0.1 M, pH 7) for $1 \mathrm{~min}$, and immersed in water overnight to remove any physisorbed polymer. Finally, the substrates were rinsed with ethanol and dried under nitrogen gas.

Characterization methods: Static and dynamic contact angle measurements were performed with the sessile drop method using an optical contact angle device equipped with an electronic syringe unit (OCA15, Dataphysics, Germany). Degassed Milli-Q quality 
water was used as the probe liquid. For each sample five successive measurements were made. For experiments on the stability of the initiator monolayers in acidic/basic solutions, the functionalized silicon surfaces were incubated in PB solutions with different $\mathrm{pH}$ values on a shaking plate. At regular times, substrates were taken out of solution, rinsed sequentially with water (to remove salt deposits) and ethanol, dried in a stream of nitrogen, and measured.

$X$-ray photoelectron spectroscopy (XPS) was used to evaluate the composition of the immobilized ATRP initiator monolayers. XPS spectra were obtained on a Quantera XPS instrument (Physical Electronics) using a monochromatized Al K $\alpha$ radiation (1486.6 eV) source with an X-ray beam diameter of $100 \mu \mathrm{m}$ and an electron take-off angle of $45^{\circ}$ relative to the sample surface. The spectrometer resolution was $0.2 \mathrm{eV}$ for the highresolution element scans and $0.8 \mathrm{eV}$ for the survey spectra. An argon ion beam neutralizer was not used to avoid damage to the labile bromine atom of the initiator molecule. Fitting of the high resolution scan of the $\mathrm{C} 1 \mathrm{~s}$ emission region was carried out using Gaussian-Lorentzian product functions. Before fitting, spectra were referenced to the value of $284.8 \mathrm{eV}$ for aliphatic carbon and corrected for non-linear Shirley background. $^{28}$

FTIR spectra were recorded using a Biorad FTS-575C spectrometer equipped with a nitrogen-cooled cryogenic mercury telluride detector (spectral resolution of $4 \mathrm{~cm}^{-1}, 1024$ scans). Background spectra were obtained by scanning a freshly cleaned silicon substrate. To investigate the dissociation behavior of the $\mathrm{pH}$-responsive polymer brushes, the grafted substrates were incubated in buffer solutions with different $\mathrm{pH}$ values for $15 \mathrm{~min}$, quickly rinsed with ethanol followed by drying under nitrogen gas, and characterized by FTIR.

The dry thickness of the grafted polymer layers was measured using a computercontrolled null ellipsometer (Plasmos SD 2002) working with a He-Ne laser $(\lambda=632.8$ $\mathrm{nm}$ ) at an angle of incidence of $70^{\circ}$. The measurements were averaged over at least 25 points at the surface of each sample (sampled area $5 \times 5 \mathrm{~mm}^{2}$ ). For the initiator monolayer and the grafted PMAA in dry state, refractive indexes of 1.460 and 1.475, respectively, were used. ${ }^{29}$ For measurements of the swollen thicknesses of the polymer brush, a Multiskop null ellipsometer (Optrel, Germany) in a polarizer-compensatorsample-analyzer (PCSA) configuration was used. The instrument was operated with a HeNe laser $(\lambda=632.8 \mathrm{~nm})$ as a light source and the angle of incidence was set to $70^{\circ}$. Measurements in liquid environment were conducted using a quartz flow cell with thin 
walls fixed at an angle of $70^{\circ}$ with respect to the sample plane. The angle of incidence of the light was set so that its path was normal to the window. Kinetic measurements were conducted by recording the ellipsometric angles every $5 \mathrm{~s}$. Both thickness and refractive index of the swollen polymer layer were extracted from the fitting of the measured ellipsometric angles $\Psi$ and $\Delta$ using a three-layer model for a flat film consisting of crystalline Si surface $(n=3.865-0.019 i)$, silicon oxide $(n=1.465)$, and polymer brush. The value of 1.333 was used as refractive index of the surrounding medium (PB solution with $50 \mathrm{mM}$ potassium phosphate). The thickness of the native silicon oxide layer was measured on freshly cleaned silicon surfaces and determined to be $1.7 \pm 0.1 \mathrm{~nm}$. The commercial software supplied with the Multiskop setup was used for the fitting of the measured ellipsometric angles. The program is based on a multi-layer model with optically isotropic layers and implements a modified Levenberg-Marquardt minimization algorithm for data inversion.

The swelling behavior of the responsive polymer layers was evaluated by in situ AFM in liquid environment using a NanoScope III Multimode setup (Veeco/Digital Instruments, Santa Barbara, CA) equipped with a liquid cell. AFM measurements were carried out in contact mode at ambient temperature using V-shaped $\mathrm{Si}_{3} \mathrm{~N}_{4}$ cantilevers with a length of $200 \mu \mathrm{m}$ (model NP, Veeco/Digital Instruments). The cantilever spring constants were determined using the thermal noise method resulting in $k=0.08 \mathrm{~N} / \mathrm{m}$. For imaging loading forces of $\sim 10 \mathrm{nN}$ at scan speeds of $10 \mu \mathrm{m} / \mathrm{s}$ were applied using optimized feedback parameters. Polymer layers were scratched with plastic tweezers and the height difference between the unscratched and scratched region was measured.

\subsection{References}

1 Coessens, V.; Pintauer, T.; Matyjaszewski, K. Prog. Polym. Sci. 2001, 26, 337-377.

2 Patten, T. E.; Xia, J. H.; Abernathy, T.; Matyjaszewski, K. Science 1996, 272, 866-868.

3 Reimhult, E.; Kumar, K. Trends Biotechnol. 2008, 26, 82-89.

4 Arinaminpathy, Y.; Khurana, E.; Engelman, D. M.; Gerstein, M. B. Drug Discovery Today 2009, 14, 1130-1135.

5 Han, X. J.; Studer, A.; Sehr, H.; Geissbuhler, I.; Di Berardino, M.; Winkler, F. K.; Tiefenauer, L. X. Adv. Mater. 2007, 19, 4466-4470.

6 Simon, A.; Girard-Egrot, A.; Sauter, F.; Pudda, C.; Picollet D'Hahan, N.; Blum, L.; Chatelain, F.; Fuchs, A. J. Colloid Interface Sci. 2007, 308, 337-343.

7 Dai, J. H.; Bao, Z. Y.; Sun, L.; Hong, S. U.; Baker, G. L.; Bruening, M. L. Langmuir 2006, 22, 4274-4281. 
Zhao, B.; Brittain, W. J. Prog. Polym. Sci. 2000, 25, 677-710. G. B.; Szleifer, I.; Tsukruk, V. V.; Urban, M.; Winnik, F.; Zauscher, S.; Luzinov, I.; Minko, S. Nature Mater. 2010, 9, 101-113.

Gong, P.; Genzer, J.; Szleifer, I. Phys. Rev. Lett. 2007, 98. Gong, P.; Wu, T.; Genzer, J.; Szleifer, I. Macromolecules 2007, 40, 8765-8773. Dong, R.; Lindau, M.; Ober, C. K. Langmuir 2009, 25, 4774-4779.

Polymer Brushes: Synthesis, Characterization, Applications; Advincula, R. C.; Brittain, W. J.; Caster, K. C.; Rühe, J., Eds.; Wiley-VCH: Weinheim, 2004.

Matyjaszewski, K.; Miller, P. J.; Shukla, N.; Immaraporn, B.; Gelman, A.; Luokala, B. B.; Siclovan, T. M.; Kickelbick, G.; Vallant, T.; Hoffmann, H.; Pakula, T. Macromolecules 1999, $32,8716-8724$.

Bao, Z. Y.; Bruening, M. L.; Baker, G. L. Macromolecules 2006, 39, 5251-5258.

Matyjaszewski, K.; Dong, H. C.; Jakubowski, W.; Pietrasik, J.; Kusumo, A. Langmuir 2007, 23, 4528-4531.

Yameen, B.; Kaltbeitzel, A.; Langner, A.; Duran, H.; Muller, F.; Gosele, U.; Azzaroni, O.; Knoll, W. J. Am. Chem. Soc. 2008, 130, 13140-13144.

Sui, X. F.; Zapotoczny, S.; Benetti, E. M.; Schon, P.; Vancso, G. J. J. Mater. Chem. 2010, 20, 4981-4993.

Parnell, A. J.; Martin, S. J.; Jones, R. A. L.; Vasilev, C.; Crook, C. J.; Ryan, A. J. Soft Matter 2009, 5, 296-299.

Benetti, E. M.; Reimhult, E.; de Bruin, J.; Zapotoczny, S.; Textor, M.; Vancso, G. J. Macromolecules 2009, 42, 1640-1647.

Biesalski, M.; Johannsmann, D.; Ruhe, J. J. Chem. Phys. 2002, 117, 4988-4994.

Houbenov, N.; Minko, S.; Stamm, M. Macromolecules 2003, 36, 5897-5901.

Konradi, R.; Ruhe, J. Macromolecules 2004, 37, 6954-6961.

Konradi, R.; Ruhe, J. Macromolecules 2005, 38, 6140-6151.

Nap, R.; Gong, P.; Szleifer, I. J. Polym. Sci., Part B: Polym. Phys. 2006, 44, 2638-2662.

Ramakrishnan, A.; Dhamodharan, R.; Ruhe, J. J. Polym. Sci., Part A: Polym. Chem. 2006, 44, 1758-1769.

Ramakrishnan, A.; Dhamodharan, R.; Ruhe, J. Macromol. Rapid Commun. 2002, 23, 612616.

Practical Surface Analysis, Auger and X-ray Photoelectron Spectroscopy; 2nd ed.; Briggs, D.; Seah, M. P., Eds.; John Wiley \& Sons: Chichester, England, 1990.

Polymer Handbook; 4th ed.; Brandrup, J.; Immergut, H. E.; Grulke, E. A., Eds.; Wiley: New York, 1999. 


\section{Chapter 5}

\section{Switching transport through nanopores with $\mathrm{pH}$-responsive polymer brushes for controlled ion permeability*}

This chapter contains the results on the functionalization of nanoporous platforms with poly(methacrylic acid) (PMAA) brush via surface-initiated ATRP. The electrochemical measurements presented in this chapter were performed with the help of Kaori Sugihara (ETH Zürich, Switzerland). The growth of the PMAA brush and its $\mathrm{pH}$-responsive behavior on the nanoporous platforms were confirmed by the characterization techniques SEM, FTIR and AFM. The swelling behavior of the pH-responsive PMAA brushes grafted only from the nanopore walls was investigated by AFM in aqueous liquid environment with $\mathrm{pH}$ value of 4 and 8. AFM images displayed open nanopores at $\mathrm{pH} 4$ and closed ones at $\mathrm{pH} 8$, which rationalizes the use of such polymer-modified porous chips as mechanical gating platforms. Ion conductivity across the nanopores was investigated with currentvoltage measurements at various $\mathrm{pH}$ values. Enhanced higher resistance across the nanopores was observed in a neutral polymer brush state (lower $\mathrm{pH}$ values) and lower resistance when the brush was charged (higher $\mathrm{pH}$ values). By adding a fluorescent dye in an environment of $\mathrm{pH} 4$ or $\mathrm{pH} 8$ at one side of the PMAA-brush functionalized nanopore array chips, diffusion across the nanopores was followed. These experiments demonstrated faster diffusion rates of the fluorescent molecules at $\mathrm{pH} 4$ (PMAA neutral state, open pores) and slower diffusion at pH 8 (PMAA charged state, closed pores) showing the potential of this technology for nanoscale valve applications.

\footnotetext{
* Parts of this chapter have been published in: de Groot, G.W.; Santonicola, M.G.; Sugihara, K.; Zambelli, T.; Reimhult, E.; Vörös, J.; Vancso, G.J. ACS Appl. Mater. Interfaces 2013, 5, 1400-1407
} 


\subsection{Introduction}

Functionalization of porous platforms with stimulus responsive polymer brush structures allows reversible controlled switching of surface properties inside micro- and nanochannels, and makes it possible to fabricate valves at these length scales. These functionalized porous platforms can be applied in biosensing, where they can provide stable devices to increase mechanical stability and lifetime for membrane protein screening. ${ }^{1}$

Stimulus responsive polymer grafts have been used with great success to engineer surfaces of materials. The switching can be triggered by an external stimulus in the environment of the material, e.g. changes in $\mathrm{pH}$, temperature, mechanical force or light. ${ }^{2}$ Surface-initiated controlled radical polymerization techniques are mostly used to synthesize these polymer brush structures, and the most frequently used is surfaceinitiated atom transfer radical polymerization (SI-ATRP). ${ }^{3}$ SI-ATRP provides an environment in which polymer brush growth is reproducible and yields robust polymer brush structures, well-defined in chain length and architecture. Grafting density can in principle be controlled by tuning the coverage of initiators attached to the substrates. ${ }^{4-7}$

Grafting stimulus responsive polymer brushes via SI-ATRP from porous platforms offers opportunities to different fields including delivery systems, lab-on-a-chip, microand nanofluidics and (bio)molecular screening. ${ }^{8}$ Especially weak polyelectrolyte brushes are interesting because they make it possible to control ion permeation through porous platforms by varying the $\mathrm{pH}$ of the surrounding solution, ${ }^{9-11}$ and can be useful in their swollen state as an alternative approach to support and span lipid bilayers over pores. ${ }^{12-}$ 17 Both these functionalities open ways to incorporate membrane proteins in the supported lipid bilayer and measure their ion channel activity for pharmaceutical relevance. ${ }^{18,19}$ This approach can create functional sensor surfaces with immobilized membrane proteins that are suitable for in vitro controlled electrochemical recording of their structure-function relationships and for label-free high-throughput screening of low molecular weight drug candidates. Another interesting possibility is the coupling of nitrilotriacetate (NTA) to polymer brushes with carboxylic acid groups via EDC/NHS activation for precise positioning of membrane proteins above the pore openings of the platforms. $^{20,21}$

Control of transport through polymeric membranes functionalized with smart polymer systems has already been described for both responsive polymer brush structures and (grafted) responsive hydrogels. ${ }^{22,23}$ It has been displayed that permeation 
of water and polymer solution through polymer brush functionalized polymeric membranes can be controlled by changing the $\mathrm{pH}$ of the surrounding environment. ${ }^{24-26}$ Besides pH-responsive polymer brush structures, also thermo-responsive polymer brushes were grafted to and from track-etched membranes. $N$-isopropylacrylamide (NIPAM) was polymerized by controlled radical polymerization techniques and the functionalized membranes were characterized with conductometric measurements below and above the lower critical solution temperature of polyNIPAM (pNIPAM) resulting in different permeabilities. ${ }^{27,28}$ More recently $\mathrm{pH}$-responsive polymer brush structures were grafted from single polymeric nanopores. Functionalized nanopores displayed a variation in transport of protons across the single pored membranes in response to a change in $\mathrm{pH}$. This $\mathrm{pH}$-responsive behavior originated from the protonation below pH 5 of the pyridine groups in the poly(4-vinyl pyridine) (PVP) brush, which resulted in a charged and swollen brush. In particular, current-voltage measurements showed that above $\mathrm{pH} 5$ (neutral brush) the ionic conductance was constant and low compared to $\mathrm{pH}$ values below pH 5 (charged brush) where the ionic conductance increased. ${ }^{29}$ The functionalization of macroporous silicon membranes with weak polyelectrolyte brushes synthesized by SI-ATRP was reported by the same group. These weak polyelectrolyte brush functionalized membranes were mainly characterized with focus on proton conductivity for fuel cell applications. ${ }^{30,31}$

The examples mentioned above demonstrate that current-voltage measurements are a convenient tool for investigating the ionic conductance of membranes functionalized with weak polyelectrolyte brushes in combination with $\mathrm{pH}$ variations. In addition, it is also reported that resistance measurements can be performed at polyelectrolyte multilayer filled nanopores, ${ }^{32}$ which is used for measuring the resistance of supported lipid bilayers spanned over these functionalized pores. ${ }^{12}$ Atomic force microscopy (AFM) is a well-known characterization technique for polymer brush structures, and applying AFM in a liquid environment makes it possible to monitor a responsive behavior on the polymer brush. ${ }^{33-36}$ The influence of the force applied by a AFM tip was investigated by force volume spectroscopy for polyethylene glycol chains anchored to a nanoring on a substrate. Applying less or more force changed the AFM image due to indentation of the AFM tip in the polymer brush structure. ${ }^{37}$ Yet another useful characterization method is fluorescence spectroscopy, which makes it possible to follow transport of fluorescent molecules from one side to the other of the membrane. ${ }^{38}$ 
Previously we reported the fast and reversible switching between polymer conformations at low and high $\mathrm{pH}$ values for $\mathrm{pH}$-responsive poly(methacrylic acid) (PMAA) brushes grafted from planar silicon surfaces. In that study the methanol content of the aqueous ATRP reaction mixture was varied to investigate further applications for controlled brush growth in confined spaces. ${ }^{10}$

Here we apply the brush growth from our previous study to several nanoporous platforms and characterize the polymerization using different techniques displaying the growth of PMAA brushes inside the nanopores. Current-voltage measurements and diffusion experiments in combination with fluorescence spectroscopy demonstrate control of transport through the functionalized pores by variations of the surrounding environment $\mathrm{pH}$.

\subsection{Results and Discussion}

5.2.1 Functionalization and characterization of nanoporous platforms with $\mathrm{pH}$ responsive poly(methacrylic acid) brushes

Supported and free-standing nanoporous silicon nitride films were functionalized with pH-responsive PMAA brushes using SI-ATRP. First, the pre-activated chips were treated by vapor phase deposition with an initiator layer of (3-(2bromoisobutyryl)propyl)dimethylchlorosilane molecules. After, SI-ATRP of sodium methacrylate was conducted to synthesize $\mathrm{pH}$-responsive PMAA brushes. SI-ATRP was performed in water/methanol 50:50 v/v reaction mixture to improve the wetting of the pore walls for polymer brush growth inside the pores. The polymerization was performed for $1 \mathrm{~h}$ at room temperature, which resulted in PMAA brushes with dry thickness of approximately $90 \mathrm{~nm}$, as measured by ellipsometry in air on planar silicon surfaces. ${ }^{10}$ After polymerization, a color change at the surface of the substrates was observed, which was a first indication of the film modification with the PMAA brush layer.

Scanning electron microscopy (SEM) was used to compare bare nanoporous films with PMAA-brush functionalized nanoporous films to confirm the presence of the polymeric layer inside the nanowells (Figure 5.1). SEM images were taken from the top surface and the cross-section, with the samples imaged in a tilted position. The images of the top sides clearly display that there is a layer of polymeric material on the functionalized nanoporous film. The pore openings are smaller in diameter and the edges of the pore openings are much smoother. The cross-sectional SEM images confirm 
that there is polymeric material inside the nanowells, and that the added layer on the functionalized nanoporous film is approximately $60 \mathrm{~nm}$ in thickness.

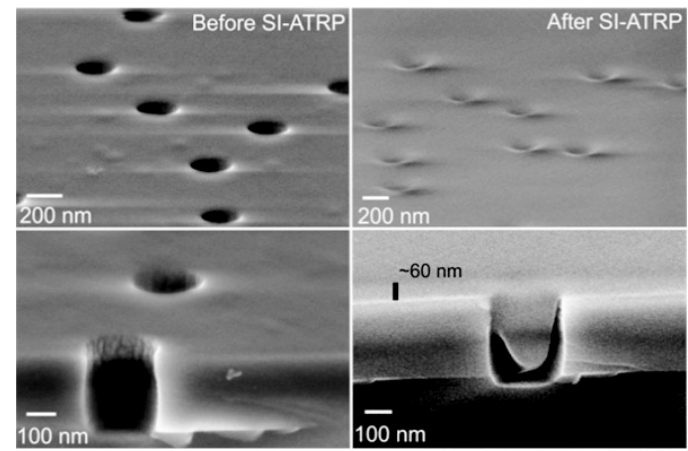

Figure 5.1: SEM images of non-functionalized (left) and PMAA-brush functionalized (right) nanoporous silicon nitride films without chrome top layer. Top images display tilted top surface views and bottom images display cross sectional views of the samples. Images on the right refer to samples after SI-ATRP and show a polymer layer stratification on top surface (smaller and smoother pore openings). From the cross sectional view a polymer layer of $\sim 60 \mathrm{~nm}$ on top surface can be estimated (vertical black line). The cross sectional view also shows smooth polymeric material inside the nanowell, in contrast with the cross sectional view of the nonfunctionalized film (left) where the rough surface of the pore wall caused by the reactive ion etching step during film preparation is evident. ${ }^{39}$

Fourier transform infrared (FTIR) spectroscopy was used to confirm the $\mathrm{pH}$-responsive behavior of the PMAA brush grafted from the nanoporous film. A functionalized nanoporous film was immersed in a phosphate solution of $\mathrm{pH} 4$ or $\mathrm{pH} 8$, and a FTIR spectrum was taken. Figure 5.2 displays the carbonyl absorption region of the PMAA brush right after immersion in each solution. The characteristic band peak of the protonated carboxylic acid groups is found at $1705 \mathrm{~cm}^{-1}$, whereas the characteristic band peak of the deprotonated carboxylic acid groups is located at $1558 \mathrm{~cm}^{-1}$. These FTIR spectra prove that PMAA brushes were grafted from nanoporous films and that these grafts respond to $\mathrm{pH}$ variations. 


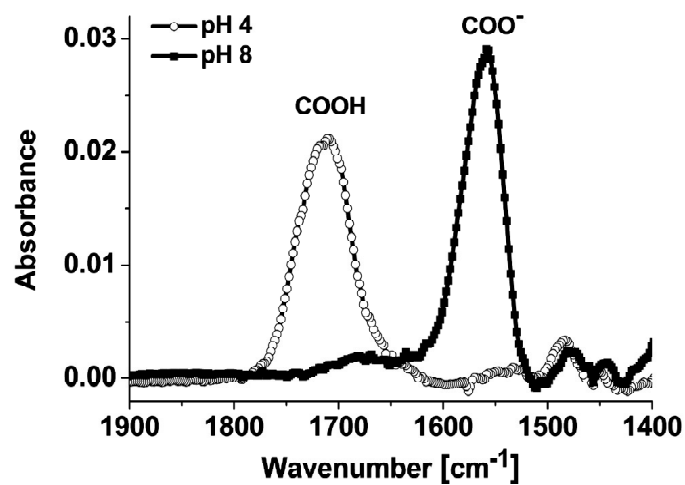

Figure 5.2: Carbonyl absorption region in FTIR spectra of a PMAA brush grafted from a nanoporous silicon nitride film after incubation in phosphate solution of $\mathrm{pH} 4 \mathrm{or} \mathrm{pH}$. FTIR spectra display protonated PMAA chains at $\mathrm{pH} 4$ and deprotonated PMAA chains at $\mathrm{pH} 8$.

AFM characterization in liquid environment was used to follow the swell and the collapse of the PMAA brushes inside the nanopores. As displayed in the SEM images of Figure 5.1, the graft layers were both grown from the wall of the nanowells as well from the top surface of the nanoporous films. The swelling of the polymer layer grafted from the top surface blocked the view at the nanopore openings by AFM. Therefore, nanoporous films with a chrome top layer were used to grow the grafts only from the silicon nitride walls of the nanowells. The chrome top layer served as a passivation layer, as there is no formation of silanol groups at the chrome surface during the activation step by piranha solution prior to the deposition of the ATRP initiator layer. It is known that chrome oxide surfaces can be functionalized by organosilanes such as trichloroalkylsilanes and triethoxyalkylsilanes. ${ }^{40,41}$ On the other hand, there are also examples in the literature where chrome is used to create patterns where silanes do not attach, including monochlorosilanes. ${ }^{42}$ To clarify this issue, water contact angle and XPS measurements were performed at silicon and chrome surfaces before and after vapor phase deposition of the initiator molecules. For silicon surfaces, contact angle values before immobilization of the initiator (right after activation in piranha solution) were $\sim 23^{\circ}$ and increased to $\sim 77^{\circ}$ after initiator immobilization, indicating the presence of the hydrophobic initiator molecules on surface. ${ }^{10}$ Chrome surfaces had a contact angle of $\sim 13^{\circ}$ after treatment in piranha solution, and $\sim 17^{\circ}$ after the deposition of the initiator molecules. These results gave a first indication that no initiator molecules were covalently linked to the chrome surface. The same silicon and chrome surfaces were investigated by XPS, and the full element analysis showed less carbon atoms and almost 
no bromine atoms at the chrome surface after treatment with the ATRP initiator as compared to the silicon surface (Table 5.1).

Table 5.1: XPS full element analysis of silicon and chrome surfaces before and after vapor phase deposition of the initiator molecules. Chrome surfaces before and after initiator deposition contain comparable levels of carbon atoms and negligible bromine atoms (bold numbers in table). Silicon surfaces after initiator deposition contained bromine atoms, whereas the control sample before initiator deposition did not. Small fractions of sodium atoms and sulfur atoms were found on the chrome surfaces as residuals from the substrate preparation.

\begin{tabular}{|c|c|c|c|c|c|c|c|}
\hline Sample & C & 0 & $\mathrm{Na}$ & Si & $S$ & $\mathrm{Cr}$ & $\mathrm{Br}$ \\
\hline $\begin{array}{l}\text { Cr before } \\
\text { initiator } \\
\text { deposition }\end{array}$ & $\begin{array}{l}6.95 \pm \\
0.43\end{array}$ & $\begin{array}{l}54.41 \pm \\
0.68\end{array}$ & $\begin{array}{l}3.51 \pm \\
0.31\end{array}$ & & $\begin{array}{l}4.23 \pm \\
0.24\end{array}$ & $\begin{array}{l}30.86 \pm \\
0.36\end{array}$ & $\begin{array}{l}0.04 \pm \\
0.02\end{array}$ \\
\hline $\begin{array}{l}\text { Cr after } \\
\text { initiator } \\
\text { deposition }\end{array}$ & $\begin{array}{l}8.26 \pm \\
0.47\end{array}$ & $\begin{array}{l}53.19 \pm \\
0.31\end{array}$ & $\begin{array}{l}3.38 \pm \\
0.29\end{array}$ & & $\begin{array}{l}3.62 \pm \\
0.38\end{array}$ & $\begin{array}{l}31.47 \pm \\
0.49\end{array}$ & $\begin{array}{l}0.07 \pm \\
0.05\end{array}$ \\
\hline $\begin{array}{l}\text { Si before } \\
\text { initiator } \\
\text { deposition }\end{array}$ & $\begin{array}{l}1.84 \pm \\
0.28\end{array}$ & $\begin{array}{l}45.48 \pm \\
0.37\end{array}$ & & $\begin{array}{l}52.68 \pm \\
0.26\end{array}$ & & & $\begin{array}{l}0.00 \pm \\
0.00\end{array}$ \\
\hline $\begin{array}{l}\text { Si after } \\
\text { initiator } \\
\text { deposition }\end{array}$ & $\begin{array}{l}12.35 \pm \\
0.75\end{array}$ & $\begin{array}{l}40.24 \pm \\
0.38\end{array}$ & & $\begin{array}{l}47.21 \pm \\
0.35\end{array}$ & & & $\begin{array}{l}0.21 \pm \\
0.10\end{array}$ \\
\hline
\end{tabular}

XPS survey spectra for a silicon and chrome surface after initiator deposition are included (Figure 5.3). From both contact angle and XPS results the conclusion was drawn that the ATRP initiator monolayer does not form on chrome surfaces when vapor phase deposition is used. At the silicon nitride surface inside the nanowells silanol groups are formed in contact with piranha solution and can therefore be used for immobilization of monochlorosilane initiator molecules. In this way it was possible to attach the initiator molecules only onto the silicon nitride surface and to graft polymer chains only from the nanopore walls. 


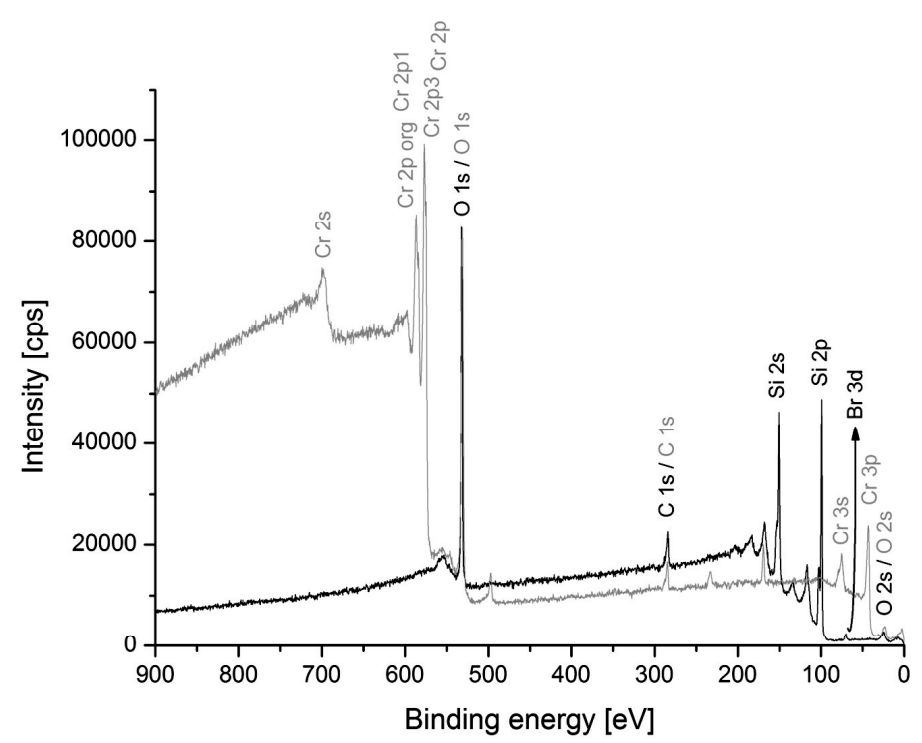

Figure 5.3: XPS survey spectra (1.5 keV) of a silicon surface (black) and a chrome surface (grey) after deposition of the ATRP initiator monolayer. The survey spectrum of the chrome surface does not show a significant bromine peak $(\mathrm{Br} 3 \mathrm{~d})$ and the area under the carbon peak (C $1 \mathrm{~s})$ is less than the area under the carbon peak (C 1s) of the initiator coated silicon surface.

These PMAA-brush functionalized nanoporous films were both characterized with SEM and AFM. With SEM, non-functionalized as well as functionalized nanoporous films were compared, with both top surface and cross-section scanned in a tilted position (Figure 5.4). It can be seen from the SEM images that the functionalized nanoporous film has polymer layers inside the nanowells. At the top surface there is a rim of polymeric material visible at the nanopore openings. This latter observation was investigated by AFM under ambient conditions. The top surface of non-functionalized and PMAA-brush functionalized nanoporous films were both scanned. On the AFM images of Figure 5.5 it can be seen that the polymer graft protrudes out of the nanopore in the case of the functionalized silicon nitride nanoporous film, whereas there is no substance around the pore rim of the non-functionalized nanoporous film. It should be noted that away from the pore rims the AFM images in Figure 5.5 show the same surface features for both the non-functionalized and the functionalized nanoporous film, and no polymer grafts attached to the chrome surface are visible. Both SEM and AFM images indicate that the passivating chrome layer method works as expected, and that there is no polymer brush grafted from the top side of the nanoporous films. 


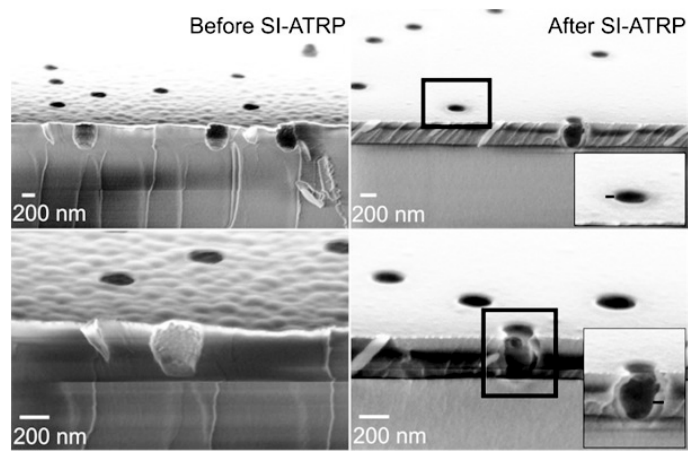

Figure 5.4: SEM images of non-functionalized (left) and PMAA-brush functionalized (right) nanoporous silicon nitride films with chrome top layer. Top images display tilted top surface and cross sectional view together, and bottom images display a zoom in at the cross sectional view. Black boxes indicate polymer grafts protruding out of the pore opening (top box, see also Figure 5.5) and polymer layers inside the nanowell (bottom box). The insets on the right show zoomed areas of the functionalized nanowells: the horizontal black lines were added to indicate the extent of the polymer layer protruding out of the pore (top inset) and along the nanopore wall (bottom inset).
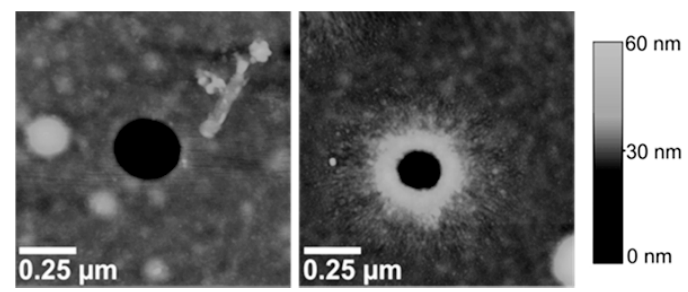

Figure 5.5: TM-AFM images (top view) in ambient environment of non-functionalized (left) and PMAA-brush functionalized (right) nanoporous silicon nitride film with chrome top layer. In the right AFM image, polymer chains grafted from the pore wall are protruding out of the pore opening.

\subsubsection{Controlled nanopore gating function by $\mathrm{pH}$-responsive poly(methacrylic acid) brushes}

In our previous study, in situ ellipsometry and AFM in liquid environment were used to investigate the swell and collapse of PMAA brushes grafted from planar surfaces upon changes of the solution $\mathrm{pH}^{10}$ This $\mathrm{pH}$-responsive behavior originates from the deprotonation of the carboxylic acids groups at higher $\mathrm{pH}$ values. The polymer chains become charged and repel each other, which results in swelling of the brushes. Besides the electrostatic interactions upon changes in $\mathrm{pH}$, the osmotic pressure of the 
counterions also plays a role on the polymer brush conformation. Large additions of salt result in the collapse of weak polyelectrolyte brushes, because of a decrease of osmotic pressure of the counterions. Conversely, low additions of salt cause an increase in brush height by exchange of protons for cations. This exchange produces an increase in the degree of dissociation and therefore swelling of weak polyelectrolyte brushes occurs. ${ }^{43}$ In the case of functionalized nanochannels, the $\mathrm{pH}$-responsive behavior of the polymer grafts can be used as a nanopore gating function. In a previous work we investigated brush thickness variations in phosphate solutions with $\mathrm{pH} 4$ and $\mathrm{pH} 8$ for PMAA grafted from planar silicon surfaces at the same conditions. Measurements by in situ ellipsometry showed large swelling in the phosphate solutions (up to $229 \mathrm{~nm}$ for $\mathrm{pH} 8$ ) and swelling factors in the range 1.1-1.3 depending on the brush grafting density. ${ }^{10}$ In the present situation, due to the different polymerization kinetics in the nanopores confined space and to the accessibility of the polymer layer conformation, it is difficult to determine brush thickness variations and corresponding swelling factors. From a theoretical study it is known that polymer chains attached close to the entrance of pores with short aspect ratios tend to protrude out of the pores in a good solvent. By protruding out of the pore into the reservoir, polymer chains relieve nanoconfinement and stretch away from the pore. ${ }^{44}$ To observe the swelling of the PMAA grafts inside the nanowells of the nanoporous silicon nitride films, AFM measurements were performed on substrates with a passivating chrome top layer (Figure 5.6). Measurements were in phosphate solution of $\mathrm{pH} 4$ (brush collapsed state) or $\mathrm{pH} 8$ (maximum brush swelling in liquid). AFM characterization was also chosen to analyze the response of the PMAA brushes grafted from the nanowells in the actual environment of the application considered in this work, that is the mechanical gating for the ion permeability control through nanopores. At both $\mathrm{pH}$ values the same area with five pores with a diameter of $200 \mathrm{~nm}$ was scanned. A pore size of $200 \mathrm{~nm}$ was chosen to completely block the pores at the maximum brush swelling conformation. In fact, our previous study on PMAA brushes grafted from planar silicon surfaces had displayed a maximum brush swelling up to 229 $\mathrm{nm}$ in phosphate solutions of $\mathrm{pH} 8 .^{10}$ Compared to the AFM image obtained in dry conditions in Figure 5.5, the polymer grafts in Figure 5.6 were already swollen by uptake of phosphate solution. It can be seen from the corresponding AFM image taken at $\mathrm{pH} 4$ that the nanopores at this $\mathrm{pH}$ value are not closed by the graft. After changing the liquid in the AFM liquid cell to phosphate solution of $\mathrm{pH} 8$ the same area was scanned again and a significant swelling of the PMAA brushes out of the pores was observed. This 
result displays the opening and closing of PMAA-brush functionalized nanopores with the chosen polymerization conditions by changing the $\mathrm{pH}$ of the surrounding environment from $\mathrm{pH} 4$ to $\mathrm{pH} 8$.

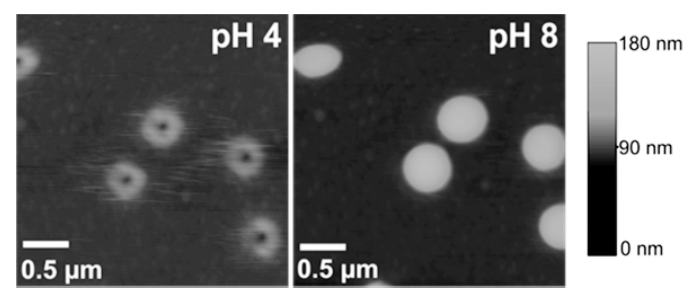

Figure 5.6: CM-AFM images (top view) of PMAA-brush functionalized nanoporous silicon nitride film with a chrome top layer in phosphate solution of $\mathrm{pH} 4$ or pH 8. At pH 4 (left) the polymer chains protruding out of the five pore openings are swollen, nevertheless the pore openings are not completely blocked. At $\mathrm{pH} 8$ (right) there is a significant swelling of the polymer chains out of the five nanowells.

To investigate the $\mathrm{pH}$-controlled gating properties of the PMAA brushes, nanopore array chips with channels accessible on both sides in a silicon nitride membrane were functionalized with SI-ATRP and analyzed in current-voltage measurements. Chips with arrays of four nanochannels with a diameter of $400 \mathrm{~nm}$ were used, and the ionic conductance was obtained at different $\mathrm{pH}$ values of the phosphate solution. Here nanochannels of $400 \mathrm{~nm}$ in diameter were used to limit entrapment of air bubbles, which would affect the conductivity measurements. At each $\mathrm{pH}$ value a current-voltage measurement was applied where the voltage was cycled between $-0.2 \mathrm{~V}$ and $0.2 \mathrm{~V}$ at a sweep rate of $10 \mathrm{mV} / \mathrm{s}$ and the current across the functionalized chip was measured (Figure 5.7). From $0 \mathrm{~V}$ to $0.2 \mathrm{~V}$ the I-V curves for all $\mathrm{pH}$ values were linear and the resistance was calculated from the slopes. The graph in Figure 5.8a shows the decrease of electrical resistance with increasing $\mathrm{pH}$, which implies that charging up the PMAA chains favors ion transport across the nanopore array chip. This behavior is in agreement with previous published work on nanochannels functionalized with PVP brushes, if we consider the different nature of the polymer brush and its opposite $\mathrm{pH}$-dependent gating properties. ${ }^{29}$ In that case, in fact, a significant decrease in the transmembrane ionic current was measured with increasing the solution $\mathrm{pH}$, that is when the PVP chains are in the neutral state. The inset in Figure 5.8a displays the result of a control experiment performed with a non-functionalized nanopore array chip with one pore 
with a diameter of $800 \mathrm{~nm}$. The same phosphate solutions with varied $\mathrm{pH}$ values were used to confirm that the change in electrical resistance over the nanopore array chip is coming from the PMAA graft. The graph in the inset shows no change in electrical resistance by variation of $\mathrm{pH}$. The reversibility of PMAA brushes between $\mathrm{pH} 4$ and $\mathrm{pH} 8$ was investigated in depth in our previous study, where the degree of dissociation by FTIR and the brush thickness by in situ ellipsometry displayed reversibility over four $\mathrm{pH}$ cycles. ${ }^{10}$ In this study, the I-V measurements were repeated in solution of various $\mathrm{pH}$ values between $\mathrm{pH} 4$ and $\mathrm{pH} 8$ in a cycle. Figure 5.8a displays the curve obtained from $\mathrm{pH} 8$ to $\mathrm{pH}$ 4. The complete cycle starting from $\mathrm{pH} 4$ to $\mathrm{pH} 8$ and back is presented Figure $5.8 \mathrm{~b}$, which shows for all $\mathrm{pH}$ values a reproducible value in electrical resistance. These results confirm that PMAA brushes are grafted from nanopore array chips and that they respond to variations of $\mathrm{pH}$ in their environment. The ionic conductance across PMAAbrush functionalized nanochannels can be controlled by varying the $\mathrm{pH}$ of the surrounding environment.

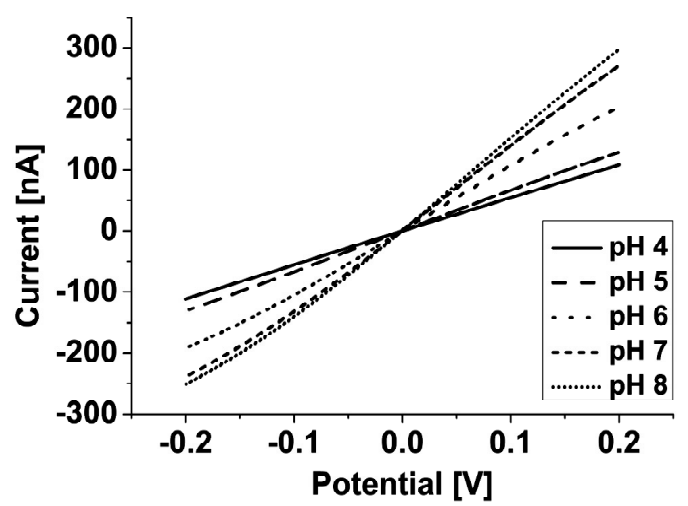

Figure 5.7: Current-voltage measurements of PMAA-brush functionalized nanopore array (4 pores of $400 \mathrm{~nm}$ in diameter) at varied $\mathrm{pH}$ values. 
a)

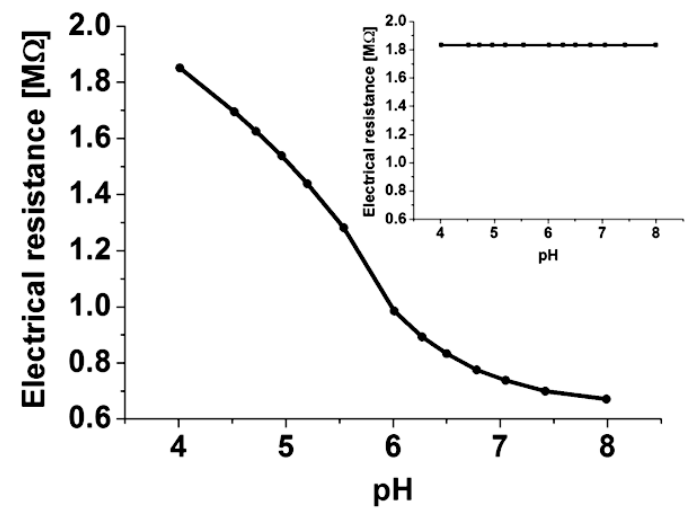

b)

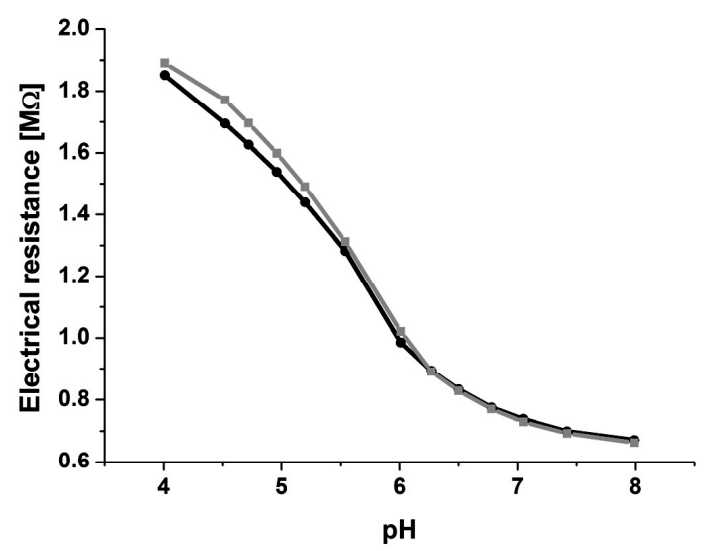

Figure 5.8: a) Change in electrical resistance of PMAA-brush functionalized nanopore array at varied $\mathrm{pH}$ values. By increasing the solution $\mathrm{pH}$, electrical resistance decreases. Inset (same axes) displays control experiment with non-functionalized nanopore array, which displays no response to variations of $\mathrm{pH}$ solution. b) A complete cycle was performed from $\mathrm{pH} 4$ to $\mathrm{pH} 8$ (grey) and back to $\mathrm{pH} 4$ (black). Comparable values of electrical resistance were obtained for each $\mathrm{pH}$ value during the cycle.

Results from the AFM measurements on PMAA grafted nanowells (Figure 5.6) clearly show that the pores are open at $\mathrm{pH} 4$ and closed at $\mathrm{pH}$ 8. To assess further the mechanical gating properties of the polymer brush in response to $\mathrm{pH}$ variations, fluorescent dye diffusion experiments were performed using functionalized chips with arrays 512 pores of $800 \mathrm{~nm}$ in diameter. Pore sizes of $800 \mathrm{~nm}$ in diameter were chosen to avoid bubble entrapment in the sieve, therefore to have a reproducible diffusion area at different solution conditions. At the same time, in the permeability measurements it 
was necessary to maximize the throughput of the diffusing fluorescent dye so that it could be measured by a fluorometer. A two-chamber setup filled with phosphate solution of $\mathrm{pH} 4$ or $\mathrm{pH} 8$ was used and the diffusion of the fluorescent dye rhodamine 6G across the chip was followed. Samples were taken after fixed time intervals and analyzed by fluorescence spectroscopy. The intensities given by fluorescence spectroscopy were used to calculate back the concentration of the samples with use of the calibration curves for $\mathrm{pH} 4$ and $\mathrm{pH} 8$. The relative concentrations of diffused rhodamine $6 \mathrm{G}$ were calculated with respect to the start concentration and are shown in Table 5.2. From this table it can be seen that the translocated fluorophore concentrations obtained at $\mathrm{pH} 4$ are higher than the ones obtained at pH 8 after $19.5 \mathrm{~h}$ or $16 \mathrm{~h}$. Changing the order in which the two phosphate solutions were applied yielded the same result. Also the $\mathrm{p} K_{\mathrm{a}}$ of rhodamine $6 \mathrm{G}$ has to be taken into account, this value is around 7.5 , which means that rhodamine $6 \mathrm{G}$ is positively charged at $\mathrm{pH} 8$. At $\mathrm{pH} 8$ the negatively charged PMAA chains and the positively charged rhodamine $6 \mathrm{G}$ molecules form ion pairs resulting in hindered diffusion across the chips. From this it is clear that the diffusion of rhodamine $6 \mathrm{G}$ in the pore with charged environment and more closed state at $\mathrm{pH} 8$ is slower due to both brush hindrance effect and rhodamine $6 \mathrm{G}$ binding to the polymer layer. These results show that mechanical gating by $\mathrm{pH}$-controlled PMAA brushes grafted from nanochannels is possible. Further analysis of the permeability data in terms of diffusion coefficients was difficult to perform for this system, due to the complicated geometry of the interface of the functionalized nanochannels and the confinement effect that plays an important role on the acid-base equilibrium of the $\mathrm{pH}$-responsive brush. ${ }^{11} \mathrm{~A}$ concentration gradient was used as the driving force for dye diffusion. At $\mathrm{pH} 8$ the swollen brush blocks the pores more than at $\mathrm{pH} 4$, resulting in hindered diffusion. In addition, the previous mentioned electrostatic interaction between rhodamine $6 \mathrm{G}$ and the charged polymer chains at $\mathrm{pH} 8$ further decreases the dye diffusion. As a result of both effects, nanochannels functionalized with PMAA brushes can be effectively used for stimuli-gated nanofiltration. 
Table 5.2: Relative concentrations of rhodamine 6G from diffusion experiments with PMAA functionalized nanopore chips (pore diameter $800 \mathrm{~nm}$ ) at $\mathrm{pH} 4$ and $\mathrm{pH} 8$. Regardless of the order of the solution applied first, slower diffusion of rhodamine $6 \mathrm{G}$ across the functionalized nanochannels is measured with solutions of $\mathrm{pH} 8$.

\begin{tabular}{lll}
\hline \multicolumn{2}{c}{ Solution $\mathbf{p H}$} & $\begin{array}{l}\text { Diffused rhodamine } 6 \mathbf{G} \\
\text { Relative concentration }\left[\% \times 10^{2}\right]\end{array}$ \\
\hline \multirow{2}{*}{ Chip 1 } & $\mathrm{pH} 4$ after 19.5 h & 12.8 \\
& $\mathrm{pH} 8$ after 19.5 h & 0.73 \\
\hline \multirow{2}{*}{ Chip 2 } & $\mathrm{pH} 8$ after 16 h & 1.82 \\
& $\mathrm{pH} 4$ after 16 h & 6.3 \\
\hline
\end{tabular}

For future functional assays for transporter membrane proteins the throughput of the nanopores must be exactly monitored with an ultimate resolution of a single channel. Therefore, permeate-brush interactions in the on- or off-state of the single nanopore must be further studied. Repetition of the previous mentioned diffusion measurements with a neutral fluorescent dye is a manner to do this, as the dye diffusion is then only influenced by the conformational changes of the brush by applying a stimulus. There are not so many neutral dyes available and we chose bodipy R6G, which is very similar as rhodamine 6G, but without charges. The diffusion experiments with this neutral dye were so far not successful due to the limited water solubility of uncharged dye molecules.

\subsection{Conclusions}

Nanoporous platforms were functionalized with pH-responsive PMAA brushes using surface-initiated ATRP. Polymer grafting from silicon nitride films with etched nanowells was confirmed by SEM and FTIR characterization. The same nanoporous films with a passivating chrome top layer were used to synthesize PMAA grafts only on the walls of the nanowells. The swell and collapse of the $\mathrm{pH}$-responsive polymer chains on the pore walls was investigated by AFM in liquid environment of $\mathrm{pH} 4$ or $\mathrm{pH}$ 8. The AFM images displayed open pores at $\mathrm{pH} 4$ and closed pores at $\mathrm{pH} 8$.

Several nanopore gating functions of PMAA-brush functionalized nanopore array chips were explored. The gating of ions could be controlled by varying the $\mathrm{pH}$ of the surrounding environment of the functionalized nanochannels. Increasing the $\mathrm{pH}$ of the surrounding environment resulted in a decrease of electrical resistance across the 
nanochannels. Mechanically gating was investigated by following the diffusion of a fluorescent dye across the functionalized nanochannels. Diffusion of the dye molecule was slower at $\mathrm{pH} 8$ when the PMAA chains are in a charged and swollen state, and therefore hindering the diffusion through the nanochannels.

The properties of the $\mathrm{pH}$-responsive PMAA-brush functionalized nanoporous platforms illustrate the potential applications in electrochemical (bio)sensors for the controlled gating of ions and in nanofluidics as valves for low molecular weight molecules.

\subsection{Experimental Section}

Materials: Allyl 2-bromo-2-methylpropionate (CAS no. 40630-82-8, 98\%), chlorodimethylhydrosilane (CAS no. 1066-35-9, 98\%), chloroplatinic acid hexahydrate (CAS no. 18497-13-7, $\geq 37.50 \%$ Pt basis), sodium methacrylate (CAS no. 5536-61-8, 99\%), CuBr (CAS no. 7787-70-4, 99.999\%), $\mathrm{CuBr}_{2}$ (CAS no. 7789-45-9, 99.999\%), 2,2'-bipyridine (CAS no. 366-18-7, $\geq 99.0 \%$ ), rhodamine 6G (CAS no. 989-38-8, dye content $~ 95 \%$ ) were purchased from Sigma-Aldrich and used without further purification. All solvents were of high purity, and deionized water from a Milli-Q purification system (Millipore Advantage A10) was used throughout. Phosphate solutions (50 mM phosphate) with various $\mathrm{pH}$ values were prepared by titrating aliquots from the same stock $(\mathrm{pH} 7.4)$ using $\mathrm{HCl}$ or $\mathrm{KOH}$ solutions.

Nanoporous silicon nitride films with pore diameters of $200 \mathrm{~nm}$ and a pore depth of $300 \mathrm{~nm}$ were prepared by colleagues of the Laboratory for Surface Science and Technology at ETH Zürich using particle lithography. ${ }^{39,45}$ The nanopores were etched in silicon nitride films supported on silicon or glass substrates, and will be referred to as nanowells. Such nanoporous films were used with or without a passivating chrome layer on the top surface. Chips with a single pore or with an array of pores accessible on both sides in a $5 \times 5 \mathrm{~mm}^{2}$ and $300 \mathrm{~nm}$ thick silicon nitride membrane were fabricated by Leister Technologies AG. ${ }^{46}$ Chips with 4 pores of $400 \mathrm{~nm}$ in diameter, 1 pore of $800 \mathrm{~nm}$ in diameter and 512 pores of $800 \mathrm{~nm}$ diameter were used. The pores of these chips will be indicated as nanochannels in this work.

Nanopore functionalization with PMAA brushes: All nanoporous platforms were functionalized combining ATRP and the grafting from approach following a previously published procedure. ${ }^{10}$ Briefly, nanoporous silicon nitride surfaces were cleaned and activated in piranha solution $\left(\mathrm{H}_{2} \mathrm{SO}_{4} / \mathrm{H}_{2} \mathrm{O}_{2} 70: 30 \mathrm{v} / \mathrm{v}\right.$ ) for 30 min (warning: piranha 
solution reacts strongly with organic compounds and should be handled with extreme caution), rinsed with water and ethanol, and dried in a stream of nitrogen. Next, a monolayer of the ATRP initiator (3-(2-bromoisobutyryl)propyl)dimethylchlorosilane was deposited on the nanoporous surfaces by vapor phase deposition, which was followed by ATRP of sodium methacrylate at room temperature for $1 \mathrm{~h}$ under argon atmosphere. The surface-initiated ATRP was performed in a water/methanol mixture 50:50 v/v to improve the wetting of the pore walls and allow for polymer brush growth inside the nanopores. Sodium methacrylate $(50 \mathrm{mmol})$ was dissolved in the ATRP medium $(10 \mathrm{~mL})$ and the solution degassed before addition to the Schlenk flask with $\mathrm{CuBr}(1 \mathrm{mmol})$, $\mathrm{CuBr}_{2}(0.1 \mathrm{mmol})$, and 2,2'-bipyridine $(2.2 \mathrm{mmol})$ under inert atmosphere. After stirring for $15 \mathrm{~min}$, the ATRP mixture was transferred to argon-filled vials with the initiatorcoated nanoporous samples. After polymerization, the nanoporous chips were washed with water and with ethylenediaminetetraacetic acid (EDTA) solution (0.1 M, pH 7), and then immersed in water overnight to remove any physisorbed polymer. Finally, chips were rinsed with ethanol and dried under nitrogen gas.

Characterization techniques: Scanning Electron Microscopy (SEM) images were taken with a HR-LEO 1550 FEF SEM in vacuum. Cross-sections of the nanoporous films were obtained by breaking the substrates after cleaning, in case of a non-functionalized nanoporous film, or after polymerization, in case of PMAA-brush functionalized nanoporous films.

Fourier transform infrared (FTIR) spectra were obtained with a Biorad FTS-575C spectrometer equipped with a nitrogen-cooled cryogenic mercury telluride detector (spectral resolution of $4 \mathrm{~cm}^{-1}, 1024$ scans). The background spectrum was obtained by recording the spectrum of a cleaned silicon nitride nanoporous film. The $\mathrm{pH}$-responsive behavior of PMAA brushes grafted from silicon nitride nanoporous films was investigated by immersing the PMAA-brush functionalized nanoporous film in a phosphate solution of $\mathrm{pH} 4$ or $\mathrm{pH} 8$ for 15 minutes, rinsing it with ethanol, drying it under nitrogen stream, and scanning by FTIR.

Static contact angle measurements were performed with the sessile drop method using an optical contact angle device equipped with an electronic syringe unit (OCA15, Dataphysics, Germany). Degassed Milli-Q water was used as the probe liquid. For each sample three successive measurements were made.

X-ray photoelectron spectroscopy (XPS) was used to evaluate the immobilization of initiator molecules on silicon surfaces and chrome surfaces. A $20 \mathrm{~nm}$ thick layer of 
chrome was evaporated on a silicon wafer to serve as a model surface. XPS spectra were obtained on a Quantera XPS instrument (Physical Electronics) using a monochromatized Al $\mathrm{K} \alpha$ radiation $(1486.6 \mathrm{eV})$ source with an X-ray beam diameter of $100 \mu \mathrm{m}$ and an electron take-off angle of $45^{\circ}$ relative to the sample surface. The spectrometer resolution was $0.2 \mathrm{eV}$ for the high-resolution element scans and $0.4 \mathrm{eV}$ for the survey spectra. An argon ion beam neutralizer was not used to avoid damage to the labile bromine atom of the initiator molecule.

Atomic force microscopy (AFM) images of non-functionalized and PMAA-brush functionalized nanoporous films with a chrome top layer were obtained under ambient conditions in tapping mode (TM-AFM) with a NanoScope III Multimode setup (Digital Instruments/Veeco-Bruker, Santa Barbara, CA, USA) using silicon cantilevers with resonance frequencies of $200-500 \mathrm{kHz}$ (type PPP-NCH-W, Nanosensors, Wetzlar, Germany) and a EV-scanner (Digital Instruments/Veeco-Bruker). The swelling behavior of the PMAA brush upon $\mathrm{pH}$ variation was evaluated by in situ AFM in liquid environment using a NanoScope III Multimode setup equipped with a liquid cell. AFM measurements were carried out in contact mode (CM-AFM) (with minimal loading force of $\sim 10 \mathrm{nN}$ using optimized feedback parameters) using commercially available V-shaped $\mathrm{Si}_{3} \mathrm{~N}_{4}$ cantilevers (model NP, $k=0.58 \mathrm{~N} / \mathrm{m}$, Digital Instruments/Veeco-Bruker).

Current-voltage measurements were performed with an Autolab PSTAT12 Instrument (Ecochemie, Utrecht, The Netherlands). Current-voltage curves were obtained between two $\mathrm{Ag} / \mathrm{AgCl}$ electrodes purchased from Lot-Oriel AG (WPI reference electrode for ECQCM Module QSP 020). Nanopore array chips were placed in a two-chamber setup and the chambers were filled with phosphate solutions of various $\mathrm{pH}$. During the measurements the two-chamber setup was placed in a Faraday cage.

Diffusion experiments with nanopore array chips functionalized with PMAA brush were performed in a two-chamber setup filled with phosphate solution of $\mathrm{pH} 4$ or $\mathrm{pH} 8$. The fluorescent dye rhodamine $6 \mathrm{G}$ was added at one side of the chip and after $19.5 \mathrm{~h}$ or $16 \mathrm{~h}$ a sample for fluorescence spectroscopy was taken. After rinsing the setup, the phosphate solution was switched to the other $\mathrm{pH}$ and rhodamine $6 \mathrm{G}$ was again added at one side of the polymer brush functionalized nanopore chip. The calibration was performed by measuring the fluorometer responses of known concentrations of rhodamine $6 \mathrm{G}$ dye molecules in phosphate solution of $\mathrm{pH} 4$ or $\mathrm{pH} 8$. Fluorescence spectroscopy was performed with a Perkin Elmer spectrometer. 


\subsection{References}

1 Reimhult, E.; Kumar, K. Trends Biotechnol. 2008, 26, 82-89.

2 Stuart, M. A. C.; Huck, W. T. S.; Genzer, J.; Muller, M.; Ober, C.; Stamm, M.; Sukhorukov, G. B.; Szleifer, I.; Tsukruk, V. V.; Urban, M.; Winnik, F.; Zauscher, S.; Luzinov, I.; Minko, S. Nat. Mater. 2010, 9, 101-113.

Barbey, R.; Lavanant, L.; Paripovic, D.; Schuwer, N.; Sugnaux, C.; Tugulu, S.; Klok, H. A. Chem. Rev. 2009, 109, 5437-5527.

4 Matyjaszewski, K.; Xia, J. H. Chem. Rev. 2001, 101, 2921-2990.

5 Luzinov, I.; Minko, S.; Tsukruk, V. V. Soft Matter 2008, 4, 714-725.

6 Ducker, R.; Garcia, A.; Zhang, J. M.; Chen, T.; Zauscher, S. Soft Matter 2008, 4, 17741786.

Nagase, K.; Kimura, A.; Shimizu, T.; Matsuura, K.; Yamato, M.; Takeda, N.; Okano, T. J. Mater. Chem. 2012, 22, 19514-19522.

8 Adiga, S. P.; Brenner, D. W. J. Funct. Biomater. 2012, 3, 239-256.

9 Tagliazucchi, M.; Azzaroni, O.; Szleifer, I. J. Am. Chem. Soc. 2010, 132, 12404-12411.

10 Santonicola, M. G.; de Groot, G. W.; Memesa, M.; Meszynska, A.; Vancso, G. J. Langmuir 2010, 26, 17513-17519.

11 Tagliazucchi, M.; Szleifer, I. Soft Matter 2012, 8, 3292-3305.

12 Sugihara, K.; Voros, J.; Zambelli, T. ACS Nano 2010, 4, 5047-5054.

13 Kumar, K.; Isa, L.; Egner, A.; Schmidt, R.; Textor, M.; Reimhult, E. Langmuir 2011, 27, 10920-10928.

14 Simonsson, L.; Gunnarsson, A.; Wallin, P.; Jonsson, P.; Hook, F. J. Am. Chem. Soc. 2011, 133, 14027-14032.

15 Roder, F.; Waichman, S.; Paterok, D.; Schubert, R.; Richter, C.; Liedberg, B.; Piehler, J. Anal. Chem. 2011, 83, 6792-6799.

16 Lazzara, T. D.; Kliesch, T. T.; Janshoff, A.; Steinem, C. ACS Appl. Mater. Interfaces 2011, 3, 1068-1076.

17 Santonicola, M. G.; Memesa, M.; Meszynska, A.; Ma, Y. J.; Vancso, G. J. Soft Matter 2012, 8, 1556-1562.

Overington, J. P.; Al-Lazikani, B.; Hopkins, A. L. Nat. Rev. Drug Discovery 2006, 5, $993-$ 996.

19 Arinaminpathy, Y.; Khurana, E.; Engelman, D. M.; Gerstein, M. B. Drug Discovery Today 2009, 14, 1130-1135.

Dai, J. H.; Bao, Z. Y.; Sun, L.; Hong, S. U.; Baker, G. L.; Bruening, M. L. Langmuir 2006, 22, 4274-4281. Sun, L.; Dai, J. H.; Baker, G. L.; Bruening, M. L. Chem. Mater. 2006, 18, 4033-4039. 
22 Tang, M.; Zhang, R.; Bowyer, A.; Eisenthal, R.; Hubble, J. Biotechnol. Bioeng. 2003, 82, 4753. Bernstein, R.; Anton, E.; Ulbricht, M. ACS Appl. Mater. Interfaces 2012, 4, 3438-3446. Ito, Y.; Inaba, M.; Chung, D. J.; Imanishi, Y. Macromolecules 1992, 25, 7313-7316. Ito, Y.; Park, Y. S.; Imanishi, Y. Langmuir 2000, 16, 5376-5381. Zhang, H. J.; Ito, Y. Langmuir 2001, 17, 8336-8340. Alem, H.; Duwez, A. S.; Lussis, P.; Lipnik, P.; Jonas, A. M.; Demoustier-Champagne, S. J. Membr. Sci. 2008, 308, 75-86. Alem, H.; Jonas, A. M.; Demoustier-Champagne, S. Polym. Degrad. Stab. 2010, 95, 327331.

Yameen, B.; Ali, M.; Neumann, R.; Ensinger, W.; Knoll, W.; Azzaroni, O. Nano Lett. 2009, 9, 2788-2793.

Yameen, B.; Kaltbeitzel, A.; Langner, A.; Duran, H.; Muller, F.; Gosele, U.; Azzaroni, O.; Knoll, W. J. Am. Chem. Soc. 2008, 130, 13140-13144. Yameen, B.; Kaltbeitzel, A.; Glasser, G.; Langner, A.; Muller, F.; Gosele, U.; Knoll, W.; Azzaroni, O. ACS Appl. Mater. Interfaces 2010, 2, 279-287. Sugihara, K.; Voros, J.; Zambelli, T. J. Phys. Chem. B 2010, 114, 13982-13987. Lin, Y. H.; Teng, J.; Zubarev, E. R.; Shulha, H.; Tsukruk, V. V. Nano Lett. 2005, 5, 491-495. Benetti, E. M.; Reimhult, E.; de Bruin, J.; Zapotoczny, S.; Textor, M.; Vancso, G. J. Macromolecules 2009, 42, 1640-1647. Sui, X. F.; Zapotoczny, S.; Benetti, E. M.; Schon, P.; Vancso, G. J. J. Mater. Chem. 2010, 20, 4981-4993. Kutnyanszky, E.; Vancso, G. J. Eur. Polym. J. 2012, 48, 8-15. Lim, R. Y. H.; Deng, J. ACS Nano 2009, 3, 2911-2918. Jovanovic-Talisman, T.; Tetenbaum-Novatt, J.; McKenney, A. S.; Zilman, A.; Peters, R.; Rout, M. P.; Chait, B. T. Nature 2009, 457, 1023-1027. Reimhult, E.; Kumar, K.; Knoll, W. Nanotechnology 2007, 18, 275303. Anac, I.; McCarthy, T. J. J. Colloid Interface Sci. 2009, 331, 138-142. Hild, R.; David, C.; Muller, H. U.; Volkel, B.; Kayser, D. R.; Grunze, M. Langmuir 1998, 14, 342-346. Fujihira, M.; Morita, Y. J. Vac. Sci. Technol. B 1994, 12, 1609-1613. Biesalski, M.; Johannsmann, D.; Ruhe, J. J. Chem. Phys. 2002, 117, 4988-4994. Peleg, O.; Tagliazucchi, M.; Kroger, M.; Rabin, Y.; Szleifer, I. ACS Nano 2011, 5, 47374747. 
46 Heyderman, L. J.; Ketterer, B.; Bachle, D.; Glaus, F.; Haas, B.; Schift, H.; Vogelsang, K.; Gobrecht, J.; Tiefenauer, L.; Dubochet, O.; Surbled, P.; Hessler, T. Microelectron. Eng. 2003, 67-8, 208-213. 


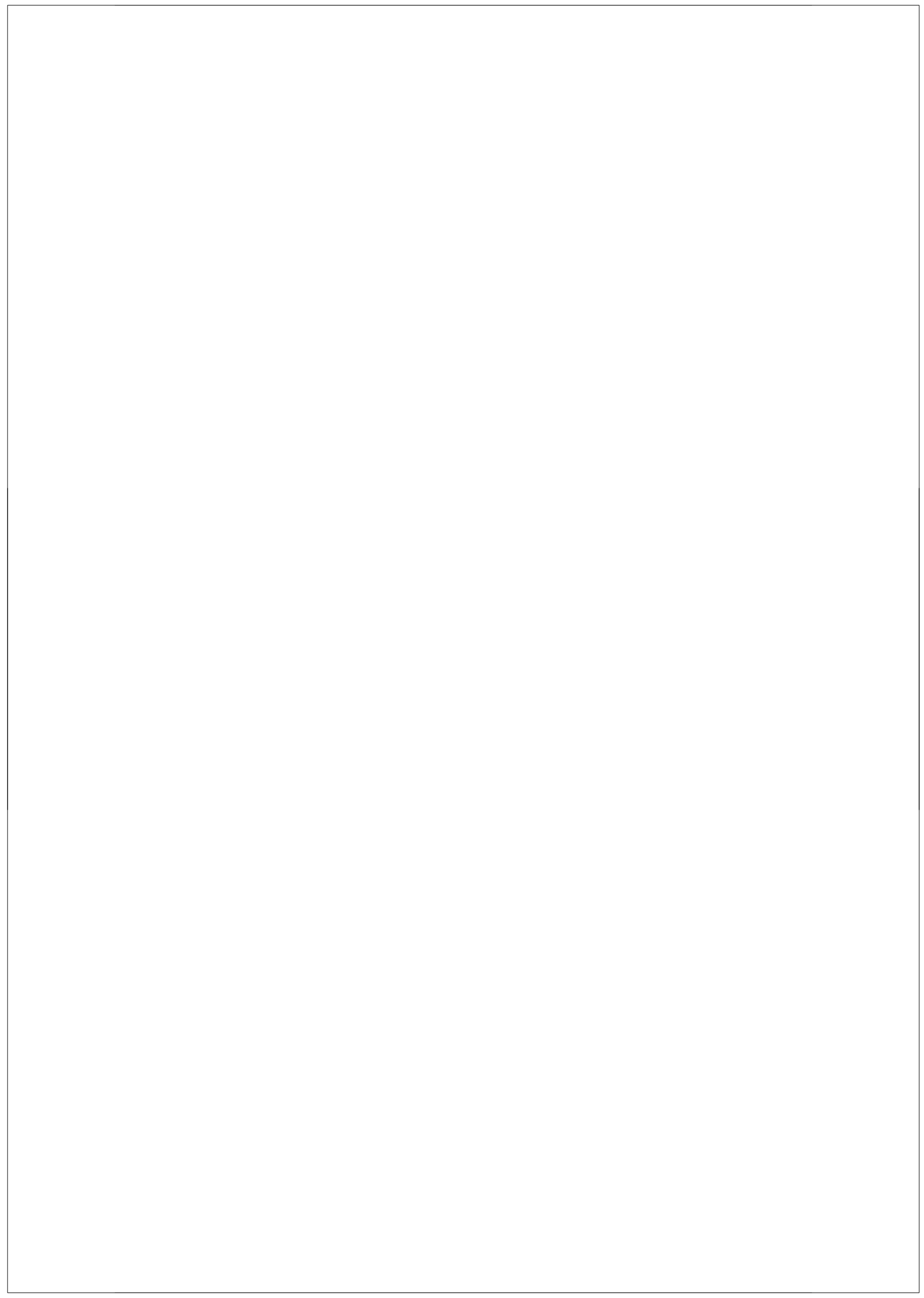




\section{Chapter 6}

Smart polymer brush structures for guiding the self-assembly of porespanning lipid bilayers with integrated membrane proteins*

In this chapter we provide a description of silicon-based nanopore array chips functionalized with $\mathrm{pH}$-responsive poly(methacrylic acid) (PMAA) brushes via surfaceinitiated atom transfer radical polymerization (SI-ATRP) and their use as supports for pore-spanning lipid bilayers with integrated membrane proteins. His-tagged proteins were selectively and reversibly bound to nitrilotriacetic acid (NTA) moieties introduced to the PMAA brush, and consequently lipid bilayer membranes were formed by liposome rupture and fusion. Enhanced membrane resistance as determined by electrochemical impedance spectroscopy (EIS) and free diffusion of dyed lipids observed as fluorescence recovery after photobleaching confirmed the presence of a lipid bilayer. Immobilization of the His-tagged membrane proteins on the NTA-modified PMAA brush near the pore edges was characterized by fluorescence microscopy. The polymer-modified integrated system allowed us to adjust the protein density in the free-standing bilayers, which were stabilized by the polymer brush in the pores. EIS measurements of reused chips also displayed a significant increase in membrane resistance, which make the chips robust platforms for membrane protein assays.

\footnotetext{
* Parts of this chapter have been submitted for publication by de Groot, G.W.; Demarche, S.; Santonicola, M.G.; Tiefenauer, L.; Vancso, G.J.
} 


\subsection{Introduction}

Membrane proteins inserted or attached to lipid bilayer membranes are the gatekeepers of cells and therefore the largest class of drug targets. More than $60 \%$ of the pharmaceutical compounds currently on the market target membrane proteins. ${ }^{1,2}$ Biosensor surfaces having pore-spanning lipid bilayers with integrated membrane proteins are expected to significantly improve the high-throughput screening of drug candidates. $^{3-5}$ In addition to pharmaceutical screening with its relevant industrial applications, the scientific investigation of the diverse structure-function relationships of membrane proteins can benefit from these membrane protein assays. ${ }^{6}$

There are many challenges in the development of biosensors for membrane protein screening. For instance the surface properties are one important factor and appropriate chemical modification of supports can enhance fusion of liposomes resulting in supported bilayers. ${ }^{6}$ Artificial lipid membranes obtained by liposome fusion are preferred over lipid membranes from painting lipids dissolved in organic solvents, as residual aliphatic molecules in the latter type might interact with the membrane proteins and impair their function. Moreover, membrane protein systems reconstituted over porous surfaces have several disadvantages. First, a solid support might impede the protein functionality by a direct contact, and secondly the accumulation of ions in the space between the bilayer and the support leads to fast saturation and causes a low sensitivity for analytical electrochemical applications. ${ }^{7}$ Silicon-based membranes with predetermined pore arrays have been used to achieve stable and functional painted lipid bilayers. ${ }^{8}$ In that work, the long-term stability of lipid bilayers was investigated for several lipids, and the bilayers functionality was confirmed by electrochemical measurement after insertion of the potassium transporter valinomycin.

Schmitt and colleagues ${ }^{9}$ reported one of the first chemical modification of a nanoporous platform to promote liposome fusion into a free-standing, solvent-free lipid bilayer with a membrane resistance too low to record single channel activity but high enough for the collective activity of ion channel ensembles. A cholesterol derivative was chemisorbed via a thiol end group to the porous alumina substrates and large unilamellar vesicles (LUVS) were fused on the surface. Recently, Korman et al. ${ }^{10}$ described nanopore-spanning lipid bilayers over a $\mathrm{Si}_{3} \mathrm{~N}_{4}$ pore array of 40000 pores. Prior to the lipid bilayer formation from rupture of small unilamellar vesicles (SUVs) of $200 \mathrm{~nm}$ in diameter, the silicon nitride membrane with pores of $130 \mathrm{~nm}$ was functionalized by an organosilane layer to render the surface hydrophobic. The membrane fluidity of the 
established lipid bilayer was assessed by fluorescence recovery after photobleaching (FRAP) and found to be comparable to similar bilayers deposited on silanized glass. A high electrical resistance (>1 G $\Omega$ ) was measured by electrochemical impedance spectroscopy (EIS). However, it is questionable if such hydrophobic nanoporous surfaces are useful as support for lipid bilayers with embedded transmembrane proteins, since the hydrophobic transmembrane domain of the proteins preferentially interacts with the hydrophobic surface.

Polymers, either physically adsorbed or covalently attached to surfaces, are generally used as cushions for supporting lipid membranes on solid supports. ${ }^{11}$ In the work by Sugihara and colleagues a lipid bilayer is formed over a pore of $800 \mathrm{~nm}$ in diameter filled with a polyelectrolyte multilayer (PEM). ${ }^{12}$ The filling prevents diffusion of the small unilamellar vesicles (50 $\mathrm{nm}$ in diameter) through the pore, and the lipid bilayer is only formed at the top side of the chip. Fusion to PEMs is promoted if oppositely charged lipids are used for the liposomes. Such polymer-supported bilayers exhibit an increased lifetime and a high membrane resistance. Moreover, the PEM is permeable for monovalent ions. The use of charged polymers to induce the rupture of lipid vesicles made of zwitterionic lipids (net charge zero) on functionalized surfaces was investigated in another work. ${ }^{13}$ In that work, a zwitterionic polymer layer of sulfobetaine methacrylate (SBMA) was covalently grafted from several surfaces by atom transfer radical polymerization, and a supported lipid bilayer was formed from fusion of DOPC liposomes. The DOPC membranes on poly(SBMA) layers showed high fluidity and stability as determined by fluorescence microscopy and AFM investigations.

Another major bottleneck in the development of membrane protein assays is to position proteins over pores, so that both sides are accessible to the aqueous compartments and proteins are not impeded by interaction with the supporting surface. Specific coupling chemistries offer an efficient way to control the location of proteins on surfaces, most notably via the selective and reversible immobilization of His-tagged proteins to NTA-modified interfaces. This approach has also been used to immobilize membrane proteins within a lipid bilayer onto several supports, allowing structural investigations ${ }^{14-16}$ or functional studies of these proteins. ${ }^{17-19}$ Due to their versatile chemistry, polymers are often chosen as interfaces for modification with NTA groups and subsequent protein immobilization. ${ }^{20}$ Despite the significant research efforts in the field, to our knowledge the successful fabrication and validation of a membrane protein 
assay with a pore-spanning lipid membrane containing membrane proteins specifically located over the pores has yet to be accomplished.

In this study we present the design and synthesis of a novel integrated system featuring the controlled immobilization of membrane proteins in free-standing lipid bilayers suspended over nanoporous silicon substrates. We first functionalize the inner walls of the nanopores with $\mathrm{pH}$-responsive poly(methacrylic acid) (PMAA) brushes and subsequently modify these with nitrilotriacetate groups. The nanopore functionalization with $\mathrm{pH}$-sensitive polymer brushes allows the opening and closing of pores on command. Chemical modification was achieved by surface-initiated atom transfer radical polymerization (SI-ATRP), a well-established and versatile controlled polymerization technique that allows for the synthesis of robust polymer architectures on surfaces. ${ }^{21,22}$ Recently, we demonstrated the functionalization of nanopore arrays with PMAA brushes using SI-ATRP, and we used the $\mathrm{pH}$-driven reversible switching of the polymer brush as a way to control ion permeability across the nanopores. ${ }^{23}$ That and other work reported by our group, ${ }^{24}$ showed that at physiological conditions negatively charged PMAA brushes swell and fill the pores. Another advantage of the functionalization with PMAA brushes is the availability of the carboxylic acid groups that can be coupled to the amine group of NTA moieties after activation by N-(3dimethylaminopropyl)- $\mathrm{N}^{\prime}$-ethylcarbodiimide hydrochloride/N-hydroxysuccinimide (EDC/NHS) chemistry. ${ }^{25}$ We use this strategy to position liposomes with integrated Histagged membrane proteins above the pores. By using nanoporous surfaces modified with stimulus responsive polymers, this study presents the next logical step towards controlled transport through membrane protein assays, which would enable a selective pharmaceutical screening of these proteins.

\subsection{Results and Discussion}

\subsubsection{Functionalization of nanopore chips with NTA-modified PMAA brushes}

The nanopore array chips have a silicon oxide surface that was first functionalized with PMAA brushes using surface-initiated ATRP of sodium methacrylate, and subsequently the carboxylic acid groups of the polymer were activated and derivatized with aminobutyl NTA via EDC/NHS chemistry (Scheme 6.1). 


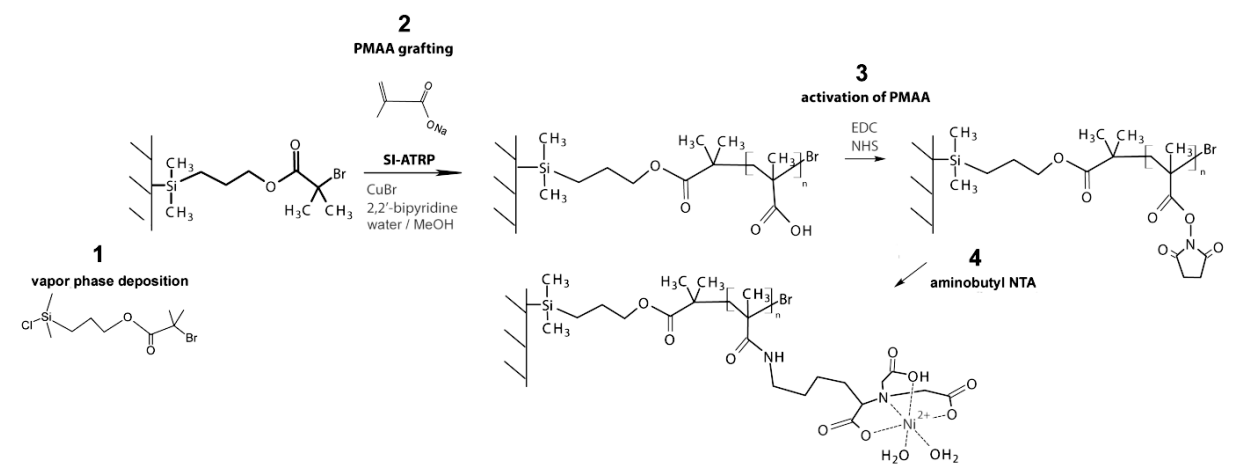

Scheme 6.1: Reaction scheme of the functionalization of silicon nanopore chips with NTAmodified PMAA brushes: (1) Immobilization of the ATRP initiator (3-(2bromoisobutyryl)propyl)dimethylchlorosilane by vapor phase deposition on the pre-activated silicon surface; (2) Synthesis of PMAA brushes by surface-initiated ATRP of sodium methacrylate; (3) Conversion of the carboxylic acid groups of PMAA into an active ester by $\mathrm{N}$-(3dimethylaminopropyl)- $\mathrm{N}^{\prime}$-ethylcarbodiimide hydrochloride (EDC) and $\mathrm{N}$-hydroxysuccinimide (NHS); (4) Reaction of the ester with aminobutyl NTA.

The PMAA brush functionalization was performed on the trans side of the nanopore chips (Figure 6.1). During vapor phase deposition of the ATRP initiator, the cis side of the chips was placed on a protecting PDMS layer. In the subsequent processes, the polymer brush is synthesized only on the trans side of the chip surface, as well as on the walls of the nanopores. This procedure was used in order to functionalize only the area around the nanopores on one side of the chips, which allows us to investigate the feasibility of the proposed concept.

During surface-initiated polymerization, nonporous silicon substrates were functionalized in parallel with the silicon nanopore chips and used for characterization of the polymer brush by various surface-sensitive methods. Typically, surface-initiated ATRP was conducted for one hour, resulting in PMAA brushes of a thickness of $177 \pm 5$ $\mathrm{nm}$, as measured by ellipsometry in air. SEM images were taken from the cis side of the nanopore chips before and after polymerization from the trans side. The SEM images of the nanopores after surface-initiated ATRP confirm that the PMAA brush is grafted from the nanopore walls all the way through to the cis side of chips (Figure 6.1). 

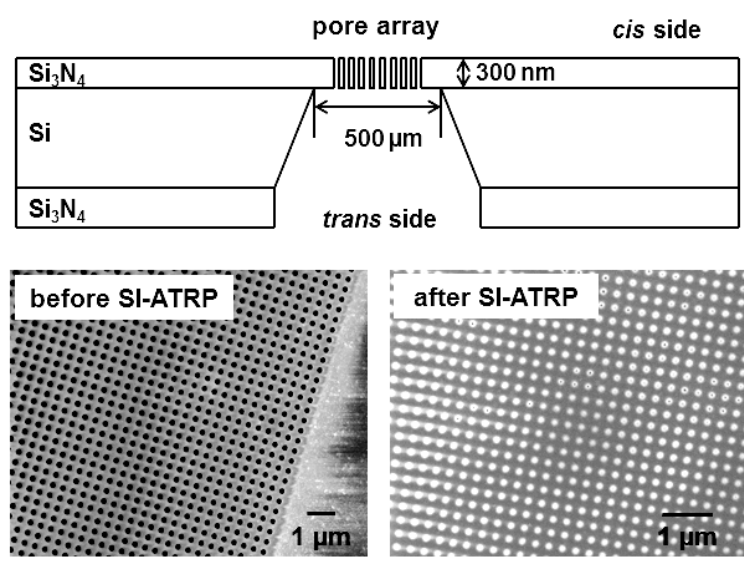

Figure 6.1: (Top) Cross-sectional scheme of the nanopore array chips used in this study. The nanopores are generated in a 300-nm-thick silicon nitride membrane with area of $0.5 \times 0.5 \mathrm{~mm}^{2}$. (Bottom) SEM images of an array chip (pore diameter $200 \mathrm{~nm}$ ) before and after surface-initiated grafting of PMAA brushes. Note: The SEM images were obtained at the cis side of the chip.

The subsequent coupling of aminobutyl NTA with the carboxylic acid groups of PMAA was confirmed by Fourier transform infrared (FTIR) spectroscopy. Figure 6.2 shows the FTIR spectra of PMAA brushes before and after NTA coupling in the carbonyl absorption region. Both spectra were obtained after immersing the sample for 15 minutes in a phosphate solution of $\mathrm{pH} 8$ to ensure that most of the carboxylic acids groups were deprotonated (as indicated by the absorbance band at $1558 \mathrm{~cm}^{-1}$ ). This allowed to collect only the absorbance signal from the characteristic peptide $\mathrm{C}=\mathrm{O}$ stretching absorbance band at $1685 \mathrm{~cm}^{-1}$ (the amide I band) arising from the coupling of NTA to the PMAA brush, eliminating any contribution from the unreacted protonated carboxylic acid groups (absorbance band at approximately $1705 \mathrm{~cm}^{-1}$ ). The spectra confirm the presence of amide groups and thus a successful coupling. Measurements of water contact angles in advancing mode on a PMAA-brush functionalized nonporous silicon surface before and after NTA coupling to the carboxylic acid groups gave values of $5.4 \pm$ $2^{\circ}$ and $6.5 \pm 3^{\circ}$, respectively, thus indicating no significant difference in the hydrophilicity of the brush-coated surfaces. 


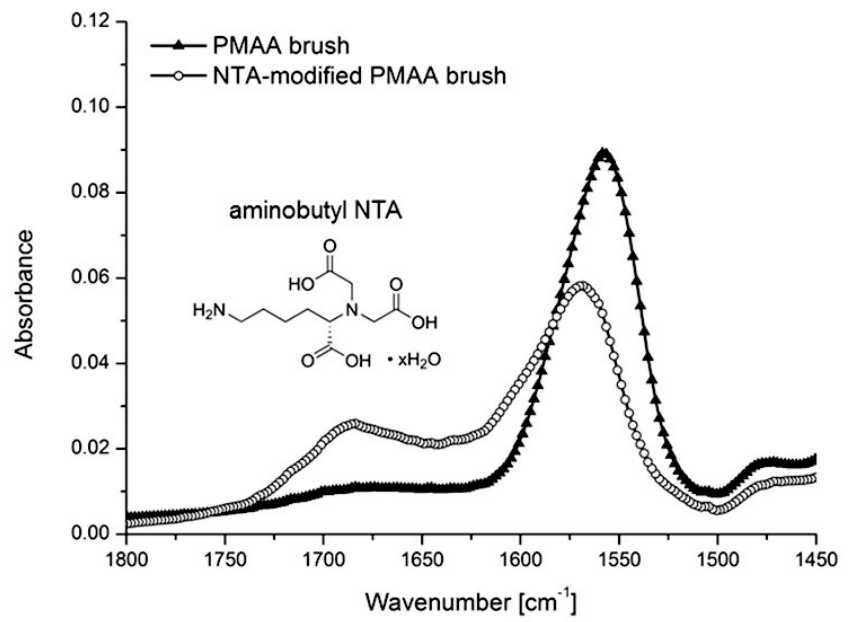

Figure 6.2: Carbonyl region of transmission FTIR absorbance spectra of a PMAA-brush functionalized nonporous silicon surface, before and after coupling with aminobutyl NTA via EDC/NHS chemistry.

To control the location of membrane proteins in the lipid membrane area suspended over the nanopores, His-tagged proteins were added to the covalently-attached NTA moieties in the presence of $\mathrm{Ni}^{2+}$ ions. The specific immobilization of a His-tagged protein to the NTA-modified PMAA brush was investigated by fluorescence confocal microscopy (Figure 6.3). A nonporous flat substrate was functionalized with PMAA brush and subsequently modified with NTA only on half of its surface. Next, it was first incubated with $\mathrm{Ni}^{2+}$ ions, followed by immersion in a solution of His-tagged green fluorescent protein (His-GFP) as a His-tagged model protein. Figure 6.3a clearly visualizes the fluorescence from His-GFP immobilized on the area where the NTA-modified PMAA brush is present. In a similar way, the immobilization of the purple His-mCherry$\mathrm{NaChBac}$ to the NTA-modified PMAA brush was verified (Figure 6.3b). By rinsing the sample with immobilized His-GFP with a solution of EDTA, the protein is released from the surface and the fluorescence intensity decreased by $92 \%$ (Figure 6.3c-d). This confirms the specific and reversible immobilization of the His-GFP proteins to NTA moieties and also highlights an efficient way to regenerate the NTA-modified polymer surface. 

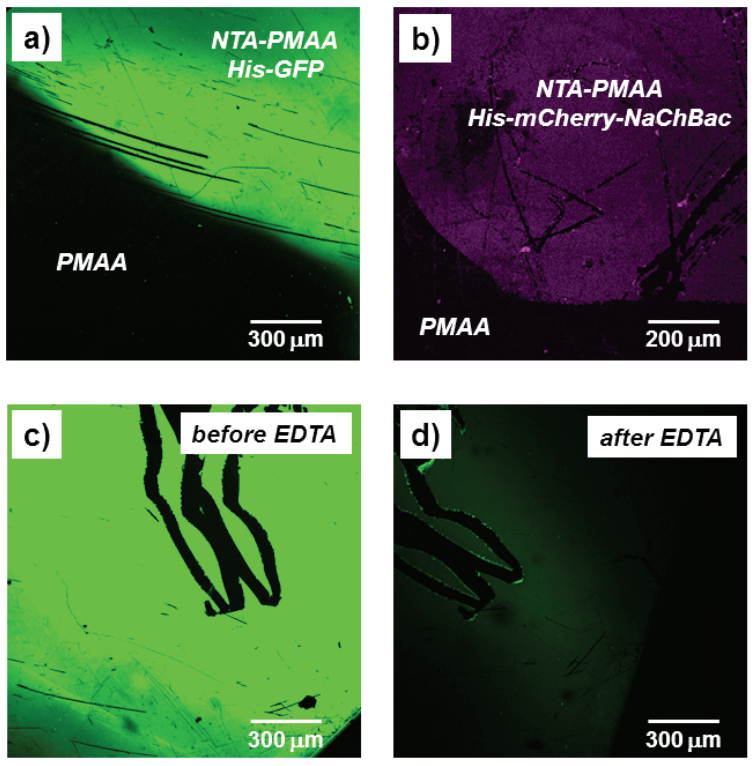

Figure 6.3: Fluorescence confocal microscopy images of PMAA brush grafted from a nonporous silicon surface with half of the surface area functionalized with NTA after incubation with (a) HisGFP and (b) His-mCherry-NaChBac. Images on the bottom are for a silicon surface functionalized with NTA-PMAA brush (c) after incubation with His-GFP and (d) after subsequent incubation in EDTA solution $(0.5 \mathrm{M})$ for $10 \mathrm{~min}$. The black pattern visible in the images is a scratch used as a reference point.

\subsubsection{Fluorescence confocal microscopy of nanopore-spanning lipid membranes}

Nanopore array chips with 16384 pores, each of $200 \mathrm{~nm}$ in diameter, were functionalized with NTA-modified PMAA brushes. The cis side of the polymer functionalized chips was incubated overnight in a suspension of POEPC/NBD-PC $(97 \% / 3 \%)$ liposomes of $50 \mathrm{~nm}$ in diameter. After the incubation, the chips were rinsed with working buffer solution and imaged by fluorescence confocal microscopy. Figure 6.4 presents fluorescence confocal measurements after incubation of the NTA-PMAAfunctionalized nanopore array with the liposomes, and the control of a polymer-free chip for comparison. The fluorescence images confirm the presence of the lipids on the cis side of the nanopore chip. In the control (Figure 6.4b) only very low fluorescence is observed, indicating that the liposomes are almost completely detached by the washing step.

The lipid membrane formation over the PMAA brush was investigated using several lipids. Best results were obtained when positively charged POEPC fused to the negatively 
charged PMAA brush at physiological pH. It should be noted that the lipid membrane only forms on the cis side of the 200-nm-wide nanopores, even when liposomes of 50 $\mathrm{nm}$ in diameter are used. In fact, at physiological pH the nanopores are completely filled with the swollen PMAA brush and not permeable to the liposomes. Previously, we had reported an investigation of the protonation of $\mathrm{pH}$-responsive PMAA brushes grafted from silicon surfaces, both neat and porous, as a function of $\mathrm{pH}$ (see also Chapter 4 and Chapter 5). ${ }^{24}$ The $p K_{a}$ value of the carboxylic acid groups of the PMAA brush was determined to be 6.5 , meaning that the polymer brush is negatively charged, and thus swollen when immersed in buffers at physiological conditions (pH 7.4). The effect of $\mathrm{pH}$ variations on the configuration of PMAA brushes grafted from nanopores had also been analyzed in AFM experiments, where it was demonstrated that the 200-nm-wide pores were closed at $\mathrm{pH}$ higher than 6.5 and open at lower $\mathrm{pH}$ values. ${ }^{23}$
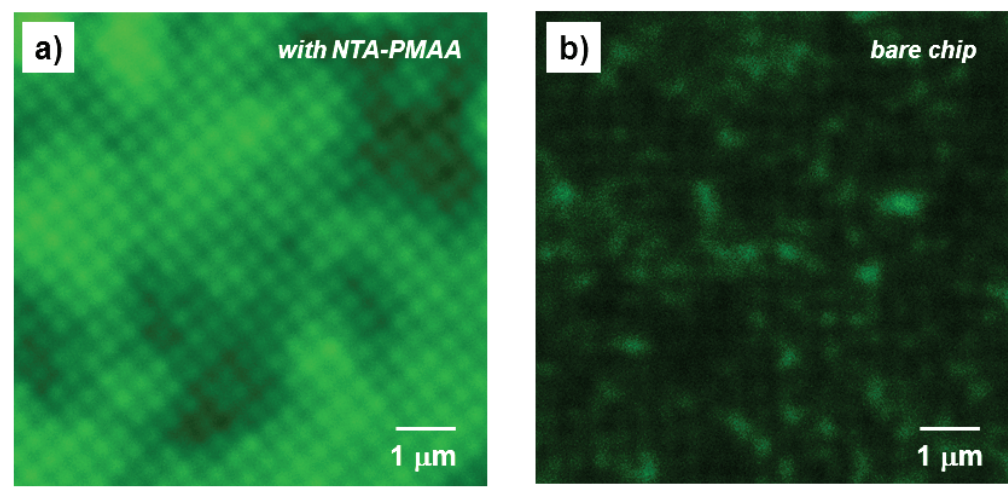

Figure 6.4: Fluorescence confocal microscopy images of a positively charged POEPC/NBD-PC $(97 \% / 3 \%)$ membrane on a nanopore array chip (cis side) with a pore diameter of $200 \mathrm{~nm}$ (a) functionalized with NTA-modified PMAA brush and (b) without the polymer brush. The brighter fluorescent areas of (a) are the pore-spanning part of the lipid membrane.

In order to confirm that liposomes have really fused and not just attached to the surface we investigated the pore-spanning membranes using fluorescence recovery after photobleaching. A circular area with a diameter of $\sim 10 \mu \mathrm{m}$ was bleached by laser and the recovery of fluorescence emission intensity, which is related to the lipid mobility within the membrane, was followed over time. Figure 6.5 shows an example of a FRAP measurement of a bilayer lipid membrane spanned over a NTA-PMAA-functionalized nanopore array chip. From the FRAP recovery curves, the diffusion coefficient $(D)$ of the labeled NBD-PC lipid was determined following the procedure of Axelrod et al. ${ }^{26}$ Three 
replicate samples were taken, and for each sample an average $D$ value was calculated from a minimum of four bleached spots in different regions of the nanopore array. The $D$ values were determined to be $0.38 \pm 0.14 \mu \mathrm{m}^{2} / \mathrm{s}, 0.32 \pm 0.14 \mu \mathrm{m}^{2} / \mathrm{s}$ and $0.12 \pm 0.04$ $\mu \mathrm{m}^{2} / \mathrm{s}$ for the three replicate samples.
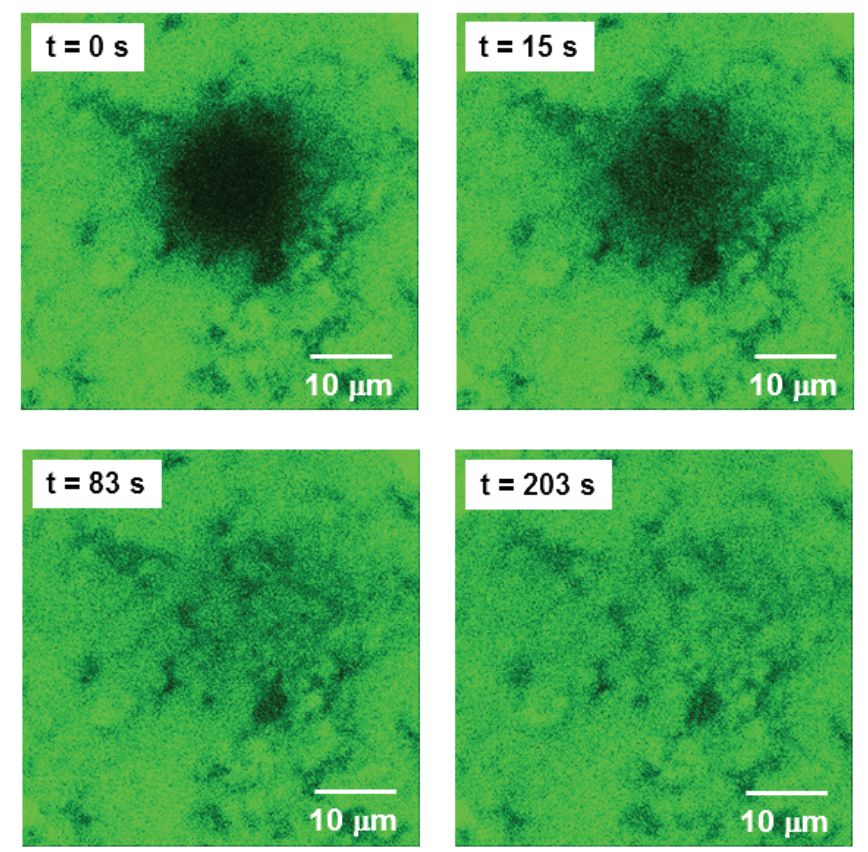

Figure 6.5: Fluorescence recovery after photobleaching (FRAP) experiment of a positively charged POEPC/NBD-PC (97\%/3\%) bilayer membrane spanned over an array of nanopores functionalized with NTA-modified PMAA brush.

The recovery of fluorescence emission intensity within the bleached area was relatively fast and completed in less than four minutes, thus confirming the presence of a continuous lipid bilayer membrane formed on the NTA-PMAA brush modified nanopores. For comparison, these $D$ values are higher than the ones obtained for lipid bilayer membranes over nanopore chips filled with polyelectrolyte multilayers $(D=0.08$ $\left.\pm 0.04 \mu \mathrm{m}^{2} / \mathrm{s}\right){ }^{12}$ and they are closer to the values reported for free lipid membranes as those of liposomes in solution $\left(D=\sim 1 \mu \mathrm{m}^{2} / \mathrm{s}\right) .{ }^{27}$ However, the values of the lipid diffusion coefficients in polymer-supported bilayers will be lower than in liposomes since the negatively charged PMAA brush interacts electrostatically with the positively charged lipids. The rate of lipid diffusion within the membrane is an important measure 
to assess its quality and usefulness for biosensor applications, as the membrane fluidity significantly relates to the activity of the reconstituted proteins. However, lipid diffusion coefficients determined on nanopore arrays should be judged with caution, as they include contribution from the free-standing part of the membrane and from the part in contact with the solid edges of the nanopores. ${ }^{28}$

\subsubsection{Electrochemical impedance spectroscopy of single-nanopore spanned lipid membranes}

In addition to FRAP measurements, electrochemical impedance spectroscopy was used to confirm lipid bilayer formation. Measurements of single-nanopore spanned lipid membranes by EIS gave information on the membrane sealing properties and electrical resistance. Silicon chips with a single pore of $200 \mathrm{~nm}$ in diameter were functionalized with NTA-PMAA brushes, charged with $\mathrm{Ni}^{2+}$ ions and then incubated with the POEPC liposomes at room temperature overnight. EIS spectra were acquired after extensive rinsing of the chips with working buffer. Before incubation in the liposome suspension, the NTA-PMAA brush functionalized chips were measured, which displayed an electrical resistance $\sim 5 \mathrm{M} \Omega$. In most cases, the total electrical resistance increased. Since both $\mathrm{pH}$ and ionic strength of the system remained constant, it can be assumed that the resistance change is due to the lipid bilayer formation on the nanopore. EIS measurements were repeated up to four hours after the end of the liposome incubation, however no further significant change in the EIS spectra was observed. Figure 6.6 shows a representative set of EIS spectra before and after the formation of the nanoporespanning lipid membrane. The bare single-pore chip has a slightly lower impedance than the chip functionalized with the NTA-modified PMAA brush. This is likely related to the presence of negative charges on the PMAA brush at the physiological $\mathrm{pH}$ values of the buffer. At the lowest frequency, the impedance can be approximated to be equal to the resistance of the functionalized pore plus the resistance of the lipid membrane. Several PMAA-brush functionalized chips were incubated overnight in the POEPC liposome solutions. The success rate of the bilayer lipid membrane formation with a resistance higher than $50 \mathrm{M} \Omega$ was $76 \%$. 


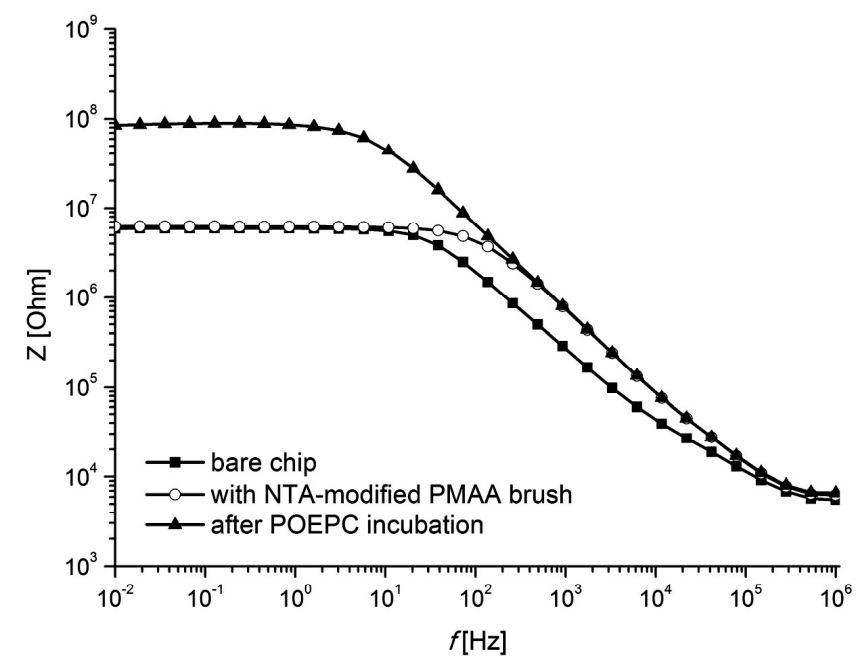

Figure 6.6: Electrochemical impedance spectra before (-o-) and after (- - -) formation of a POEPC lipid membrane on a chip with a single pore of $200 \mathrm{~nm}$ in diameter. The solution of POEPC liposomes was incubated over the nanopore chip at room temperature overnight.

\subsubsection{Nanopore-spanning lipid bilayers with integrated His-tagged membrane proteins}

The integration of membrane proteins into preformed lipid bilayers and with both sides of the protein accessible to aqueous compartments is still generally considered to be difficult to achieve and thus a limiting factor in the development of biosensors for membrane proteins. In our concept a proteolipid membrane is formed on top of a NTAfunctionalized nanoporous support, which allows for the immobilization of His-tagged membrane proteins near the pore edges. Two different protocols were investigated. The first method consisted of the immobilization of the His-NaChBac protein onto the NTAmodified PMAA brush of the nanopores array chip and subsequent liposome addition to form the lipid membrane. The His-mCherry-NaChBac was delivered to the nanopore chips in buffer used for protein purification. This buffer contains detergent (DDM, 0.03\% $\mathrm{w} / \mathrm{v}$ ) commonly used to solubilize membrane proteins in aqueous solutions while maintaining their 3D structure and functionality. ${ }^{29-31}$ After protein incubation, the solution on the cis side of the nanopores was replaced by a solution of POEPC liposomes containing fluorescent TopFluor-PC $(3 \% \mathrm{~mol})$ and left overnight. Following extensive rinsing with working buffer, the nanopore chips were imaged by fluorescence confocal microscopy to detect the fluorescent signals from both components, the protein and the lipid of the proteolipid membrane. Excitation at $488 \mathrm{~nm}$ and emission at 503-523 nm showed the TopFluor-labeled lipids, whereas excitation at $543 \mathrm{~nm}$ and emission at 550- 
$650 \mathrm{~nm}$ allowed the detection of the mCherry-labeled protein. Images by fluorescence microscopy in Figure 6.7 clearly show that both the His-tagged membrane proteins and the lipid membrane are integrated on the nanopore array chip.

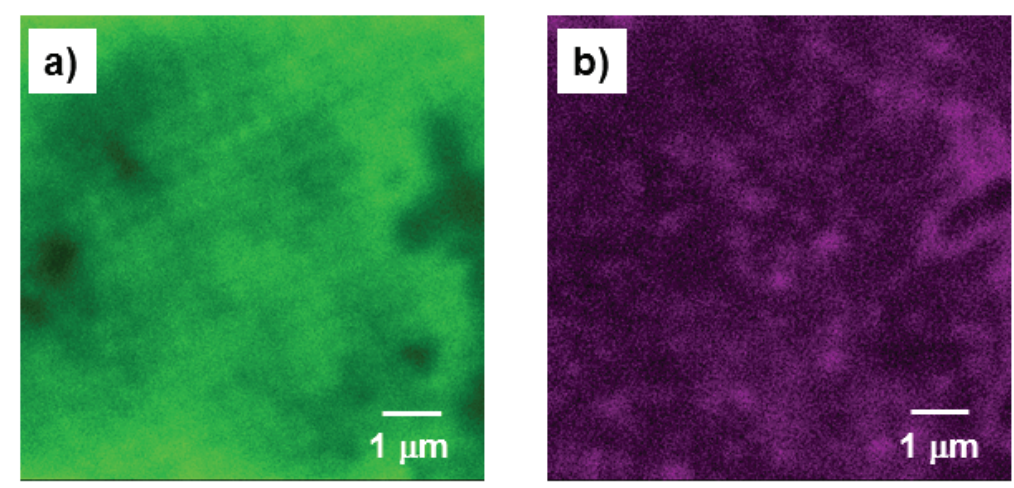

Figure 6.7: (a) Fluorescent confocal image of the lipid component POEPC/TopFluor-PC (97\%/3\% by mol) of a proteolipid membrane spanned over NTA-PMAA brush functionalized nanopores. (b) Fluorescence emission of the His-mCherry-NaChBac protein embedded in the nanoporespanning membrane.

Furthermore, FRAP measurements allow us to compare the fluidity of the lipid membrane containing the His-NaChBac protein to that of the protein-free POEPC membrane. The lipid diffusion coefficient $D$ for the proteolipid membrane decreased from $0.27 \pm 0.14 \mu \mathrm{m}^{2} / \mathrm{s}$ (no protein present) to $0.22 \pm 0.07 \mu \mathrm{m}^{2} / \mathrm{s}$ when the protein was incubated at a concentration of $25 \mu \mathrm{g} / \mathrm{mL}$, and to $0.06 \pm 0.03 \mathrm{um}^{2} / \mathrm{s}$ when the protein was incubated at $250 \mu \mathrm{g} / \mathrm{mL}$. As expected, the presence of proteins in the lipid membrane lowers its fluidity. This is ascribed to the hydrophobic and electrostatic interactions between the embedded membrane proteins and the surrounding lipids, which decreases the lipid mobility. This effect is more pronounced as more protein is included in the pore-spanning lipid membrane. However, the obtained $D$ values are still consistent with the presence of a lipid bilayer. ${ }^{32}$ Pore-spanning lipid membranes were formed on single-pore chips with immobilized His-NaChBac proteins and analyzed by electrochemical impedance spectroscopy. EIS spectra revealed an increase in electrical resistance of the chips after membrane formation up to $97 \mathrm{M} \Omega$, confirming the formation of the lipid membrane adjacent the immobilized membrane proteins.

To further simplify the process and to reduce the risk of membrane protein denaturation during immobilization onto the nanopore chip, we followed a second 
protocol and formed the suspended proteolipid membrane by direct rupture of preformed proteoliposomes of POEPC containing the His-tagged membrane protein $\operatorname{KvAP}(1 / 200 \mathrm{w} / \mathrm{w})$. The successful integration of the protein in the proteoliposomes was earlier assessed by recording the ion channel activity in a black lipid membrane. After incubation of the proteoliposomes over the nanopore chips modified with Ni-NTA-PMAA brush, the EIS spectra showed a lipid bilayer resistance over $90 \mathrm{M} \Omega$ in at least $50 \%$ of the experiments, thus confirming a proteolipid membrane formation over the polymerfunctionalized pore arrays directly from fusion of proteoliposomes. At the beginning of the study, a third protocol was followed, namely by immobilization of the His-tagged membrane proteins embedded in mixed micelles of lipids and detergent, and the subsequent removal of the detergent molecules by Bio-beads ${ }^{\mathrm{TM}}$. However, the incubation of these proteoliposomes before FRAP measurements was not successful. In the future these proteoliposomes with embedded His-tagged membrane proteins can be the ideal candidates for lipid bilayer formation by rupture and fusion including the controlled location of the proteins on the NTA-PMAA brush functionalized nanopore array chips.

One of the significant advantages of our concept is that the sensor surface can be regenerated. This was investigated with the nanopore chips modified with the NTAPMAA brush. Chips with pore-spanning lipid membranes without proteins were regenerated by cleaning with ethanol solvent; chips with membrane proteins were cleaned with EDTA solution $(0.5 \mathrm{M})$ and protein cleaning solution containing pepsin. EIS measurements of reused nanopore chips showed that a pore-spanning lipid membrane with electrical resistance of $100 \mathrm{M} \Omega$ was obtained even after seven times of use of the same chip also when incubated with proteins.

\subsection{Conclusions}

In summary, we have shown that silicon nanopore chips functionalized with NTAmodified PMAA brush offer a useful platform for free-standing lipid membranes with integrated membrane proteins. On the nanopores array, the immobilization of the Histagged membrane proteins was controlled using the NTA moiety present on the polymer brush. In physiological buffer the $\mathrm{pH}$-responsive PMAA brush $\left(\mathrm{p} K_{\mathrm{a}}=6.5\right)$ was swollen and filled the pores, thus favoring the formation of free-standing POEPC membranes. The lipid membrane was fluid and with electrical resistance up to $142 \mathrm{M} \Omega$, as demonstrated in FRAP and EIS experiments. 
An optimal procedure for the formation of the proteolipid membrane was found by immobilization of the His-tagged protein on the NTA-modified polymer brush followed by direct rupture of the POEPC liposomes. It was also demonstrated that the protein density could be varied in a wide range without impairing the formation of the lipid bilayer. This approach offers several advantages over previously reported pore-spanning lipid membranes: (1) it enhances the probability of suspending organic solvent-free POEPC lipid bilayers from liposome fusion on the nanoporous surface and (2) enables the controlled location of membrane proteins integrated in lipid membranes; (3) the covalent grafting of PMAA brush via surface-initiated polymerization resulted in a universal platform for His-tagged membrane protein assays and (4) allowed repeated use of the nanopore chips; (5) this design could be particularly useful for measuring the activity of ion channels at conditions where the PMAA brush is in its charged (swollen) state and the nanopores are permeable to ions.

The electrical resistance achieved by the integrated sensor platform of this investigation was in the best case one order of magnitude lower than the electrical resistance of painted bilayers. Nevertheless, our current platform will allow for optical activity measurements of membrane proteins, for which the resistance is not a critical parameter. In the future, several factors, including pore shape and diameter as well as the use of lipid mixtures, will be investigated to increase the electrical resistance of the membrane protein assay and enable electrochemical activity measurements.

\subsection{Experimental Section}

Materials: Allyl 2-bromo-2-methylpropionate (98\%), chlorodimethylhydrosilane, chloroplatinic acid hexahydrate ( $\geq 37.50 \%$ Pt basis), CuBr (99.999\%), 2,2'-bipyridine ( $\geq$ 99.0\%), N-(3-dimethylaminopropyl)- $\mathrm{N}^{\prime}$-ethylcarbodiimide hydrochloride (EDC, $\geq 98.0 \%$ ), $\mathrm{N}$-hydroxysuccinimide (NHS, 98\%) and $\mathrm{N \alpha}, \mathrm{N \alpha}$-bis(carboxymethyl)-L-lysine hydrate (aminobutyl NTA, $\geq 97.0 \%$ ) were purchased from Sigma-Aldrich and used without further purification. Sodium methacrylate (99\%) was purchased from Sigma-Aldrich and washed with toluene and dried under vacuum before use. 4-(2-hydroxyethyl)piperazine-1ethane-sulfonic acid (HEPES), potassium chloride $(\mathrm{KCl})$, sodium chloride $(\mathrm{NaCl})$, sodium phosphate monobasic $\left(\mathrm{NaH}_{2} \mathrm{PO}_{4}\right)$ and nickel chloride $\left(\mathrm{NiCl}_{2}\right)$, and protein cleaning solution for electrodes (HI 7073L, HANNA Instruments Inc., USA) were also purchased from Sigma-Aldrich. N-dodecyl- $\beta$-D-maltopyranoside (DDM) was purchased from Affymetrix (Santa Clara, USA). His-tagged green fluorescent protein (His-GFP) was 
purchased from BioVision (San Francisco, USA). 1-palmitoyl-2-oleoyl-sn-glycero-3ethylphosphocholine (POEPC), 1-palmitoyl-2-(12-[(7-nitro-2-1,3-benzoxadiazol-4yl)amino]dodecanoyl)-sn-glycero-3-phosphocholine (NBD-PC) and 1-palmitoyl-2(dipyrrometheneboron difluoride)undecanoyl-sn-glycero-3-phosphocholine (TopFluorPC) were purchased from Avanti Polar Lipids (Alabaster, USA). Working buffers with 10 $\mathrm{mM}$ HEPES and $150 \mathrm{mM} \mathrm{KCl}$ at $\mathrm{pH} 7.4$ were prepared in Milli-Q water (Millipore Corporation, Darmstadt, Germany) and filtered through a $0.2 \mu \mathrm{m}$ filter. All solvents were of high purity, and deionized water from a Milli-Q purification system (Millipore Advantage A10) was used throughout. Phosphate solutions (50 mM phosphate) with a $\mathrm{pH}$ value of 8 were prepared by titrating a stock solution $(\mathrm{pH} \mathrm{7.4)} \mathrm{using} \mathrm{KOH}$ solution.

Nanopore Functionalization with PMAA Brushes: Chips with an array of pores or with a single pore accessible on both sides in a $0.5 \times 0.5 \mathrm{~mm}^{2}$ window with a 300 -nm-thick silicon nitride membrane were fabricated by Leister Process Technologies (Axetris). ${ }^{33}$ Chips with 16384 pores (pitch $0.4 \mu \mathrm{m}$ ) of $200 \mathrm{~nm}$ in diameter and 1 pore of $200 \mathrm{~nm}$ in diameter were used. Nanopore array chips were functionalized combining ATRP and the "grafting from" approach following a previously published procedure. ${ }^{24}$ Briefly, chips were cleaned and activated in piranha solution $\left(\mathrm{H}_{2} \mathrm{SO}_{4} / \mathrm{H}_{2} \mathrm{O}_{2} 70: 30 \mathrm{v} / \mathrm{v}\right)$ for $30 \mathrm{~min}$ (warning: piranha solution reacts strongly with organic compounds and should be handled with extreme caution), rinsed with water and ethanol, and dried in a stream of nitrogen. Next, a monolayer of the ATRP initiator (3-(2bromoisobutyryl)propyl)dimethylchlorosilane was deposited on the pore walls by vapor phase deposition via the trans side of the chips, while protecting the cis side by placing it on a polydimethylsiloxane (PDMS) layer. The SI-ATRP of sodium methacrylate was performed at room temperature for $1 \mathrm{~h}$ under argon atmosphere. Polymerization was performed in a water/methanol mixture 50:50 v/v to improve the wetting of the pore walls and to allow for polymer brush growth inside the nanopores. Sodium methacrylate $(50 \mathrm{mmol})$ was dissolved in the ATRP medium $(10 \mathrm{~mL})$ and the solution degassed before addition to the Schlenk flask with $\mathrm{CuBr}(1 \mathrm{mmol})$ and 2,2'-bipyridine $(2.2 \mathrm{mmol})$ under inert atmosphere. After stirring for $15 \mathrm{~min}$, the ATRP mixture was transferred to the argon-filled vials with the initiator-coated nanopore chips. After polymerization, the chips were washed with water and with EDTA solution $(0.1 \mathrm{M}, \mathrm{pH}$ ), and then immersed in water overnight to remove any physisorbed polymer. Finally, chips were rinsed with ethanol and dried under nitrogen gas. 
Coupling of Aminobutyl NTA to PMAA Brushes: The activation of the carboxylic acid groups of the PMAA brushes was performed via EDC/NHS chemistry. ${ }^{34,35}$ PMAA-brush functionalized nanopore chips were immersed for 30 minutes in a $0.1 \mathrm{M}$ EDC/NHS solution (1:1 ratio) in Milli-Q water, rinsed subsequently with Milli-Q water and ethanol, and dried in nitrogen flow. Next, the chips with activated PMAA brushes were immersed for $1 \mathrm{~h}$ in a solution of aminobutyl NTA (0.1 M), adjusted to $\mathrm{pH}$ 10-10.2 with a $10 \mathrm{M} \mathrm{NaOH}$ solution, rinsed with Milli-Q water and dried in nitrogen flow.

Protein Expression and Purification: His-NaChBac and His-mCherry-NaChBac were expressed and purified using a published protocol, ${ }^{36}$ with the following modifications. His-NaChBac was genetically modified to carry a mCherry fluorescent protein at its Cterminus, yielding a His-mCherry-NaChBac protein. Gel filtration was performed using a Superdex 200 (GE Healthcare, Freiburg, Germany). His-NaChBac and His-mCherry$\mathrm{NaChBac}$ were stored at $-80{ }^{\circ} \mathrm{C}$ in gel filtration buffer $\left(50 \mathrm{~mm} \mathrm{NaH} \mathrm{PO}_{4}, 300 \mathrm{~mm} \mathrm{NaCl}\right.$, $10 \%$ glycerol and $0.03 \% \mathrm{DDM}$ at $\mathrm{pH}$ 8). His-KvAP protein was purified as previously described. $^{37}$

Preparation of (Proteo)Liposomes and Incubation: Lipids in chloroform were mixed to the desired ratio in a test tube and chloroform was evaporated under a nitrogen stream ( $>3 \mathrm{~h}$ ). Lipids were then hydrated with working buffer ( $>1 \mathrm{~h})$ to a final lipid concentration of $10 \mathrm{mg} / \mathrm{mL}$ and vortexed. Lipid solutions were extruded 31 times through a polycarbonate filter (Nucleopore Track Etched membranes, GE healthcare) of the desired liposome diameter: $800 \mathrm{~nm}, 400 \mathrm{~nm}, 200 \mathrm{~nm}$ or $50 \mathrm{~nm}$. Liposome solutions were stored at $4{ }^{\circ} \mathrm{C}$ and used within few days. His-KvAP proteoliposomes (protein/lipid ratio of $1 / 200(w / w))$ were formed using $100 \%$ POEPC liposomes of $50 \mathrm{~nm}$ in diameter. ${ }^{37}$ The functionalized nanopore chips were clamped in between two identical poly(methyl methacrylate) (PMMA) compartments of $200 \mu \mathrm{L}$ with a silicon sealing ring with an aperture exposing the pore array to the compartments. Both compartments were also open at the top side of the module and were filled with working buffer. The cis side of the chips was incubated in a $\mathrm{NiCl}_{2}$ solution $(20 \mathrm{mM}$ ) for $10 \mathrm{~min}$ and rinsed 15 times with working buffer. If proteins were incubated in the experiment, the cis side of the chips was rinsed with gel filtration buffer, incubated for $1 \mathrm{~h}$ with the proteins at the desired concentration, and then rinsed with gel filtration buffer. Liposomes or proteoliposomes solutions were diluted to a final concentration of $1 \mathrm{mg} / \mathrm{mL}$ and incubated in the cis compartment overnight. Before EIS measurements the compartment was rinsed extensively (30 times) with working buffer. 
Characterization of PMAA Brushes: Water contact angle measurements were performed with the sessile drop method, in static or advancing mode, using the OCA15 instrument (Dataphysics Instruments $\mathrm{GmbH}$, Filderstadt, Germany) equipped with an electronic syringe unit. Milli-Q water was used as the probe liquid and for each sample three successive measurements were made. Polymer brush thickness was measured using a variable-angle spectroscopic ellipsometer (VASE) (J.A. Woollam Co., Lincoln, NE, USA) in the range from 1.5 to $4.5 \mathrm{eV}$ with a step size of $0.1 \mathrm{eV}$. The measurements were performed at three different incident angles $\left(65^{\circ}, 70^{\circ}\right.$ and $\left.75^{\circ}\right)$, and were averaged over an area of $2 \mathrm{~mm}^{2}$. The ellipsometry spectra, i.e. $\psi$ and $\Delta$ as a function of the wavelength, were analyzed using the software package CompleteEASE (Woollam), employing the tabulated dielectric functions for both silicon and silicon oxide as the substrate. SEM images were taken with a JEOL JSM-6330F field emission instrument in vacuum. FTIR spectra were obtained with a Bio-Rad FTS-575C spectrometer equipped with a nitrogencooled cryogenic mercury telluride detector (spectral resolution of $4 \mathrm{~cm}^{-1}, 1024$ scans). The background spectrum was obtained by recording the spectrum of a cleaned silicon substrate. The PMAA brush grafted from silicon was investigated by immersing the sample in a phosphate solution of $\mathrm{pH} 8$ for 15 minutes, rinsing it with ethanol, drying it under nitrogen stream, and placing it in the FTIR spectrometer. The same procedure was repeated after coupling of NTA to the carboxylic acid groups of the PMAA brush.

Characterization of Pore-Spanning (Proteo)Lipid Membranes: EIS spectra were acquired using an Autolab PGSTAT 12 (Ecochemie, Utrecht, The Netherlands) equipped with a FRA module. Two $\mathrm{Ag} / \mathrm{AgCl}$ electrodes were placed in each PMMA compartment (WPI reference electrode, Lot-Oriel AG, Germany). EIS spectra were recorded from 1 $\mathrm{MHz}$ to $0.01 \mathrm{~Hz}$ at $0 \mathrm{~V}$ offset potential applying a signal amplitude of $10 \mathrm{mV}$. EIS measurements were performed in a Faraday cage. Confocal fluorescence microscopy and FRAP investigations were performed with a Leica microscope TCS SP5 (Leica Microsystems, Wetzlar, Germany) equipped with an Ar laser (488 nm), a He-Ne laser $(543 \mathrm{~nm})$, and a $63 \mathrm{X}$ glycerol objective (Leica Microsystems). Liposomes were prepared with POEPC and NBD-PC or TopFluor-PC as fluorophores (97\%/3\% by mol). After liposome incubation, the chips were rinsed 30 times with working buffer and placed in a Ludin chamber (Life Imaging Services, Basel, Switzerland) mounted with a sample coverslip of thickness \#1 (Menzel-Gläser, Germany). Images were analyzed with FRAP wizard from the LAS AF software (Leica Microsystems). From the recovery curves, lipid 
diffusion coefficients were calculated according to the theory developed by Axelrod et al. ${ }^{26}$ Fluorescent images were acquired using the same setup.

\subsection{References}

1 Overington, J. P.; Al-Lazikani, B.; Hopkins, A. L. Nat. Rev. Drug Discovery 2006, 5, 993996.

2 Rask-Andersen, M.; Almén, M. S.; Schiöth, H. B. Nat. Rev. Drug Discovery 2011, 10, 579590.

3 Bally, M.; Bailey, K.; Sugihara, K.; Grieshaber, D.; Vörös, J.; Städler, B. Small 2010, 6, 2481-2497.

4 Reimhult, E.; Kumar, K. Trends Biotechnol. 2008, 26, 82-89.

5 Zagnoni, M. Lab Chip 2012, 12, 1026-1039.

6 Tiefenauer, L.; Demarche, S. Materials 2012, 5, 2205-2242.

7 Demarche, S.; Sugihara, K.; Zambelli, T.; Tiefenauer, L.; Vörös, J. Analyst 2011, 136, 10771089.

Han, X. J.; Studer, A.; Sehr, H.; Geissbuhler, I.; Di Berardino, M.; Winkler, F. K.; Tiefenauer, L. X. Adv. Mater. 2007, 19, 4466-4470.

9 Schmitt, E. K.; Nurnabi, M.; Bushby, R. J.; Steinem, C. Soft Matter 2008, 4, 250-253.

10 Korman, C. E.; Megens, M.; Ajo-Franklin, C. M.; Horsley, D. A. Langmuir 2013, 29, 44214425.

11 Tanaka, M.; Sackmann, E. Nature 2005, 437, 656-663.

12 Sugihara, K.; Vörös, J.; Zambelli, T. ACS Nano 2010, 4, 5047-5054.

13 Santonicola, M. G.; Memesa, M.; Meszyńska, A.; Ma, Y. J.; Vancso, G. J. Soft Matter 2012, 8, 1556-1562.

14 Gupta, S.; Dura, J. A.; Freites, J. A.; Tobias, D. J.; Blasie, J. K. Langmuir 2012, 28, 1050410520.

Rigler, P.; Ulrich, W.-P.; Vogel, H. Langmuir 2004, 20, 7901-7903.

Todorovic, S.; Verissimo, A.; Wisitruangsakul, N.; Zebger, I.; Hildebrandt, P.; Pereira, M. M.; Teixeira, M.; Murgida, D. H. J. Phys. Chem. B 2008, 112, 16952-16959.

17 Ataka, K.; Giess, F.; Knoll, W.; Naumann, R.; Haber-Pohlmeier, S.; Richter, B.; Heberle, J. J. Am. Chem. Soc. 2004, 126, 16199-16206. Nowak, C.; Santonicola, M. G.; Schach, D.; Zhu, J. P.; Gennis, R. B.; Ferguson-Miller, S.; Baurecht, D.; Walz, D.; Knoll, W.; Naumann, R. L. C. Soft Matter 2010, 6, 5523-5532.

19 Roder, F.; Waichman, S.; Paterok, D.; Schubert, R.; Richter, C.; Liedberg, B.; Piehler, J. Anal. Chem. 2011, 83, 6792-6799.

Zhen, G.; Falconnet, D.; Kuennemann, E.; Vörös, J.; Spencer, N. D.; Textor, M.; Zürcher, S. Adv. Funct. Mater. 2006, 16, 243-251. 
21 Barbey, R.; Lavanant, L.; Paripovic, D.; Schuwer, N.; Sugnaux, C.; Tugulu, S.; Klok, H. A. Chem. Rev. 2009, 109, 5437-5527.

22 Matyjaszewski, K.; Xia, J. H. Chem. Rev. 2001, 101, 2921-2990.

23 de Groot, G. W.; Santonicola, M. G.; Sugihara, K.; Zambelli, T.; Reimhult, E.; Vörös, J.; Vancso, G. J. ACS Appl. Mater. Interfaces 2013, 5, 1400-1407.

24 Santonicola, M. G.; de Groot, G. W.; Memesa, M.; Meszynska, A.; Vancso, G. J. Langmuir 2010, 26, 17513-17519. Wang, C.; Yan, Q.; Liu, H.-B.; Zhou, X.-H.; Xiao, S.-J. Langmuir 2011, 27, 12058-12068. Axelrod, D.; Koppel, D. E.; Schlessinger, J.; Elson, E.; Webb, W. W. Biophys. J. 1976, 16, 1055-1069. Jacobson, K. Cell Motil. Cytoskeleton 1983, 3, 367-373.

28 Weiskopf, D.; Schmitt, E. K.; Kluhr, M. H.; Dertinger, S. K.; Steinem, C. Langmuir 2007, 23, 9134-9139.

Privé, G. G. Methods 2007, 41, 388-397.

30 Santonicola, M. G.; Lenhoff, A. M.; Kaler, E. W. Biophys. J. 2008, 94, 3647-3658.

31 Seddon, A. M.; Curnow, P.; Booth, P. J. Biochim. Biophys. Acta 2004, 1666, 105-117.

32 Lalchev, Z. I.; Mackie, A. R. Colloids Surf. B 1999, 15, 147-160.

33 Heyderman, L. J.; Ketterer, B.; Bachle, D.; Glaus, F.; Haas, B.; Schift, H.; Vogelsang, K.; Gobrecht, J.; Tiefenauer, L.; Dubochet, O.; Surbled, P.; Hessler, T. Microelectron. Eng. 2003, 67-68, 208-213.

34 Dai, J. H.; Bao, Z. Y.; Sun, L.; Hong, S. U.; Baker, G. L.; Bruening, M. L. Langmuir 2006, 22, 4274-4281.

35 Sun, L.; Dai, J. H.; Baker, G. L.; Bruening, M. L. Chem. Mater. 2006, 18, 4033-4039.

36 Studer, A.; Demarche, S.; Langenegger, D.; Tiefenauer, L. Biosens. Bioelectron. 2011, 26, 1924-1928.

37 Hutter, I.; Müller, E.; Kristiansen, P. M.; Kresak, S.; Tiefenauer, L. Microfluid. Nanofluid. 2013, 14, 421-429. 


\section{Chapter 7}

\section{Future developments to design membrane protein assays}

\subsection{Outlook}

This thesis describes solutions to problems related to the design, synthesis, characterization and testing of integrated, pore-spanning biomembrane systems, ultimately for label-free, high-throughput drug screening applications (see Chapter 3). One of the specific research objectives within the complex program to which the thesis belonged has been related to controlled reversible switching of small-molecule permeability through nanopores using stimulus responsive polymers. Permeability switching in single nanopores is already a complex issue, but in order to achieve this objective of high-throughput screening still a great amount of work is needed. However, many aspects that contribute to achieving this objective have been successful, and specific contributions by this thesis are summarized in a separate section. Here we would like to provide some ideas regarding possible options for continuation, and mention issues, which deserve further in-depth attention.

Firstly, we would like to elaborate on the issue of choosing a stimulus responsive polymer, which would allow membrane pore switching. In the thesis we describe research on using poly(methacrylic acid) (PMAA)-based brushes, which respond to $\mathrm{pH}$, thus changes in the surrounding medium. Such responses are difficult to control for individual single nanopores, therefore the system studied cannot be multiplexed. This means that permeability could be controlled at the ensemble level and not through individual pores. Therefore polymers are needed, which can be addressed at the single pore level, i.e. they must be 'nanotechnology compatible', nanoscale addressable structures, to implement in a high-throughput screening device. Single-chain addressable polymers can respond to local electric signals, as these can be channeled at the nanoscale via nanofabricated, conducting electrodes. Thus to achieve this original objective, one must build on the achievements of this thesis but choose such singlechain addressable polymers for brush growth in the nanoporous platforms. A collaboration with colleagues to investigate the potential of redox-responsive poly(ferrocenylsilanes) $(P F S)^{1}$ for pore switching was explored. We reached the 
conclusion that surface-initiated polymerizations of high grafting density brushes consisting of PFS derivatives pose a challenge, specifically as the standard atom transfer radical polymerization (ATRP) approaches applied here cannot be employed. Also other surface attached initiators explored by our collaborators posed unexpected chemical challenges. Thus, for multipore switching and permeability control by electric potential, first the fundamental problems of PFS brush growth must be solved.

The original design would have required precise positioning of membrane proteins over the pores embedded in the platform. ${ }^{2}$ Ideally the membrane protein structure and functionality should not be affected by the supporting lipid bilayer, or by the 'device'. This objective again poses numerous challenges, of which only some have been successfully achieved in the project. We have reported on the successful preparation of robust platforms consisting of PMAA brush modified nanopores in combination with the integration of His-tagged membrane proteins in artificial suspended lipid bilayers (see Chapter 6). Protein integration was demonstrated, but activity of and controlled transport through the membrane protein channels still needs to be demonstrated. In addition, for the device we targeted in the program, controlled integration of a variation of membrane proteins would be needed, which necessitates molecular fabrication of platforms with exact positioning of individual proteins at the nanoscale in the preselected positions over the array of the device.

For drug screening purposes the throughput of the nanopore array chips must be exactly monitored with an ultimate resolution of a single array pore. This again poses many physical as well as device related challenges. At the single nanopore level, for example, the throughput mechanisms depending on the on- or off-state of the stimulus responsive coating within the pores must be studied for the types of molecules one wishes to screen. In this work we achieved a demonstrator showing that $\mathrm{pH}$-responsive switching for a charged model fluorophore was possible. However, permeate-brush interactions in the on- or off-states of the single nanopore must be further studied. This also leads to problems, as for example due to the limited water solubility of uncharged dye molecules, we have had difficulties by measuring pore permeability (see Chapter 5). Selective trafficking of molecular cargo through switchable nanopores holds in general many fundamental and practical challenges. ${ }^{3}$

The above examples are related to the principles of device function, yet do not touch on the durability and reversibility of a device. While we demonstrated multiple switching with PMAA brushes (see Chapter 4), we have not tackled the stability and multiple 
switching of our platform with pore-spanning biomembranes. Obviously, before addressing this issue, all the above challenges must be solved.

In conclusion, we believe that a number of issues have been solved with success, which demonstrates the feasibility of our concepts. Yet a number of fundamental and technical problems remain open. These problems need to be studied if progress is to be made with the development of devices that would allow one to perform quantitative drug screening in real-life applications. If this could be achieved, one would be able to realize a so far unachieved objective of the pharmacological industry, i.e. the development of drugs that target membrane proteins and designed with the benefit of a membrane protein structure. ${ }^{4}$

\subsection{References}

Eloi, J.-C.; Chabanne, L.; Whittell, G. R.; Manners, I. Mater. Today 2008, 11, 28-36.

2 Tiefenauer, L.; Demarche, S. Materials 2012, 5, 2205-2242.

3 Jovanovic-Talisman, T.; Tetenbaum-Novatt, J.; McKenney, A. S.; Zilman, A.; Peters, R.; Rout, M. P.; Chait, B. T. Nature 2009, 457, 1023-1027.

4 Rask-Andersen, M.; Almen, M. S.; Schioth, H. B. Nat. Rev. Drug Discovery 2011, 10, 579590. 


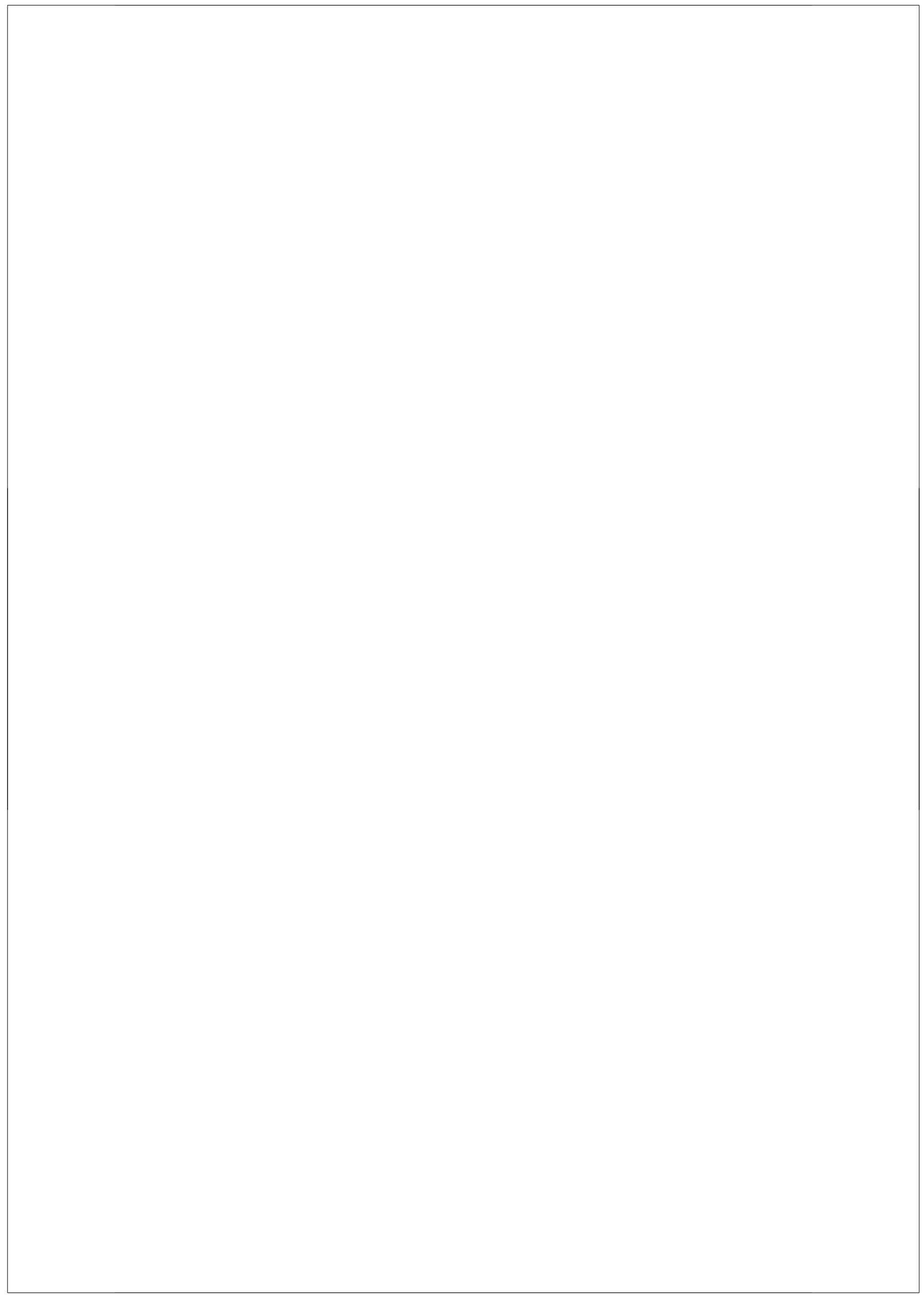




\section{Summary}

The aim of this thesis was the development of stimulus responsive polymer brush structures as micropore switches for molecular screening. This was achieved by grafting $\mathrm{pH}$-responsive poly(methacrylic acid) (PMAA) brushes from nanoporous platforms that were then integrated in functional assays for membrane proteins. Several properties of the smart PMAA brush contributed to the functionality of such protein assays. Controlled transport of ions and molecules through nanopores, support of porespanning artificial lipid bilayers and positioning of membrane proteins over the pores were achieved with the explored PMAA-brush functionalized platforms.

Chapter 1 provides a short introduction to and motivation for the control of molecular transport in bioassays with macromolecular nanotechnology. The scope of the thesis is also presented in this chapter.

In Chapter 2 a literature overview of smart polymer brushes in nanopores for controlled molecular gating is discussed, including theory, synthesis and characterization of polymer brushes. In addition, examples of stimulus responsive polymer brushes applications in surface functionalization and control of transport across pores are described.

As additional review of the literature background for the work presented in this thesis, an overview on pore-spanning lipid bilayers for functional assays of membrane proteins is presented in Chapter 3. The role of membrane proteins as drug targets is introduced and the current limitations in the development of such assays are discussed. The formation and characterization of artificial lipid bilayers and the integration of membrane proteins in the biomembranes is also part of this chapter. Specific examples of the relevance of the polymer support for artificial lipid bilayers are mentioned.

As part of the design of the modification of nanoporous platforms with polymer brushes, PMAA brushes were grafted from flat silicon surfaces using surface-initiated atom transfer radical polymerization (SI-ATRP). This is described in Chapter 4. Growth kinetics of the $\mathrm{pH}$-responsive polymer layers were investigated in mixtures of water and methanol with different ratios. The $\mathrm{pH}$-induced swelling and collapse of the brush were characterized by in situ ellipsometry, and showed a fast and reversible switching of the PMAA layers with swelling factors that increased by decreasing the methanol content of the atom transfer radical polymerization mixture. Fourier transform infrared (FTIR) spectra recorded in titration experiments indicated that the effective $\mathrm{p} K_{\mathrm{a}}$ of the PMAA 
films were shifted to the value of $6.5 \pm 0.2$ from the bulk $p K_{a}$ value of PMAA. The shift is due to ion confinement effects in the dense brush, and brings the conformational switching of PMAA brushes closer to physiological conditions. This is relevant for biologically oriented applications in miniaturized devices with rapid response times.

In Chapter 5 the PMAA brush functionalization and characterization of nanoporous platforms with wells (dead-end pores) or channels (pores through) is described. The brushes were synthesized by SI-ATRP in a mixture of water and methanol (1:1 by volume) to improve the wettability of the pores. The PMAA brush growth and its $\mathrm{pH}$ sensitive behavior on the nanoporous platforms were confirmed by scanning electron microscopy (SEM), atomic force microscopy (AFM) and FTIR spectroscopy. AFM imaging was further used to observe the swelling of the PMAA brush selectively inside the wells upon varying the $\mathrm{pH}$ of the liquid environment from 4 to 8 . AFM images showed open nanopores at $\mathrm{pH} 4$ and closed ones at $\mathrm{pH}$ 8. Platforms with nanochannels were functionalized with PMAA brushes and investigated by cyclic voltammetry at varied $\mathrm{pH}$ values. The functionalized pores had higher electrical resistance when the brush was in a neutral state and lower resistance when the brush was charged. The results of these measurements, combined with diffusion experiments using a fluorescent dye to investigate mechanical gating, demonstrated the controlled transport of ions and small molecules across the pores. All the results presented in this chapter rationalize the use of nanoporous platforms functionalized with PMAA brushes for controlled gating applications, for example as electrochemical (bio)sensors or nanoscale valves.

In Chapter 6, a description is provided discussing functionalization of silicon-based nanopore array chips functionalized with PMAA brush, which were used as supports for pore-spanning lipid bilayers. The formation of the suspended artificial lipid bilayers was achieved by spontaneous liposome rupture and fusion. Enhanced membrane resistance as determined by electrochemical impedance spectroscopy (EIS) and free diffusion of dyed lipids observed as fluorescence recovery after photobleaching (FRAP) confirmed the presence of the lipid bilayer. Besides for the support of the lipid bilayer, the PMAA chains were functionalized with nitrilotriacetic acid (NTA) for immobilization of Histagged membrane proteins. The localization of the membrane proteins near the pore edges and their integration in the pore-spanning lipid bilayer was imaged by fluorescence microscopy. It was also demonstrated that the protein density could be varied in a wide range without impairing the formation of the lipid bilayer. Although improvements are needed for the electrochemical measurements of membrane protein 
activity, yet the potential application of the integrated platform for ion channel protein assays is demonstrated.

An outlook with a discussion of the current limitations to design screening devices for membrane proteins is in Chapter 7. Future developments of the work presented in this thesis include: addressing the switch on/off of individual pores for controlled transport across selected pores; analyzing the transport of ions and low-molecular-weight drug candidates per single nanopore; and test the universal applicability of the designed assay platforms by integrating different types of membrane proteins. 


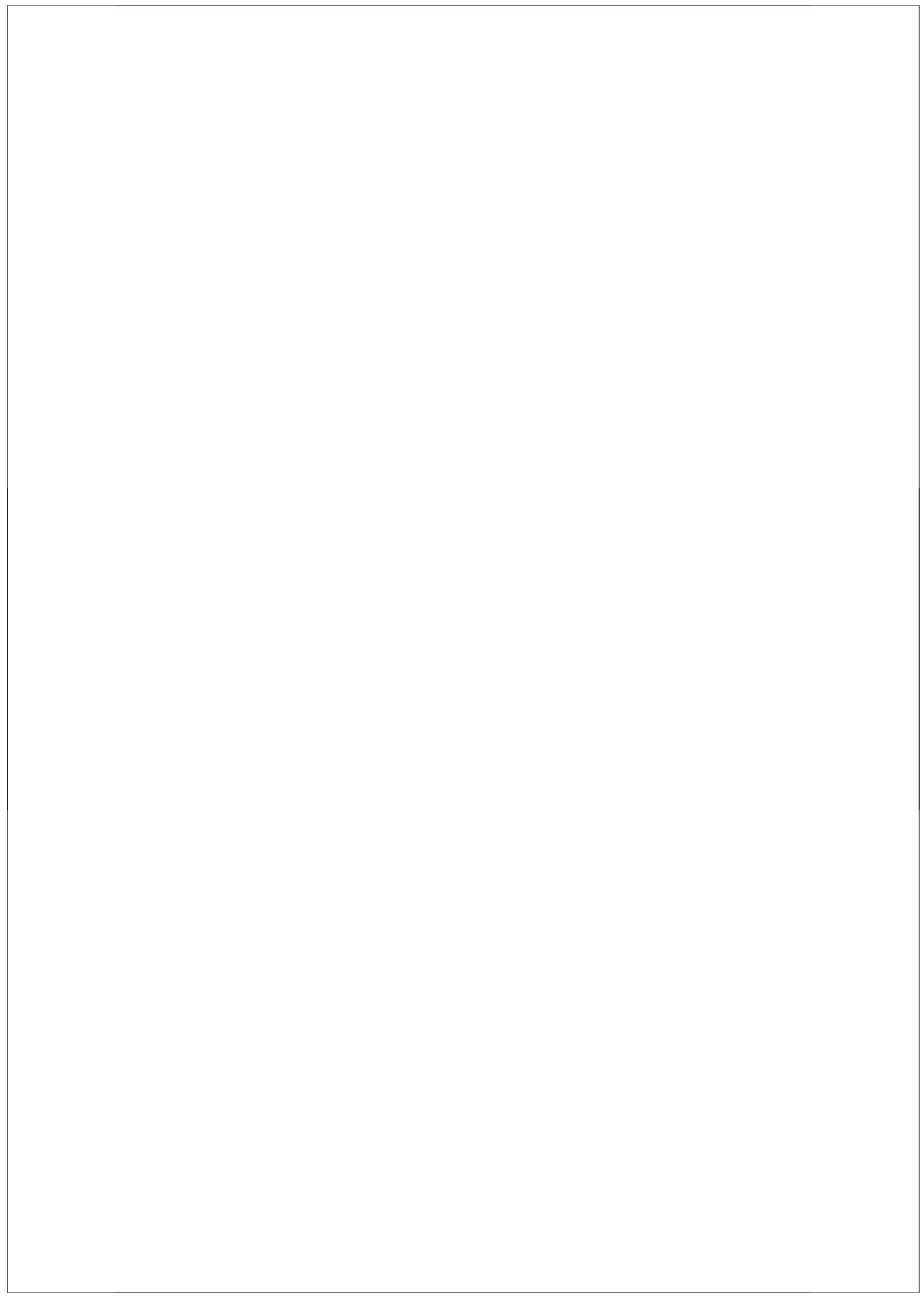




\section{Samenvatting}

Het doel van het onderzoek beschreven in dit proefschrift is de ontwikkeling van stimulus responsive polymeerborstels als kleppen in microporiën voor moleculaire analyse. Dit is bereikt door polymerisatie van $\mathrm{pH}$-responsive poly(methacrylzuur) (PMAA) borstels vanaf nanoporeuze platformen, om deze platformen vervolgens te integreren in functionele analyses voor membraaneiwitten. Verscheidene eigenschappen van de intelligente PMAA borstel dragen bij aan de functionaliteit van deze membraaneiwitanalyses. Met de in dit proefschrift onderzochte PMAA borstel gefunctionaliseerde platformen zijn gestuurd transport van ionen en moleculen door nanoporiën, dragen van porie-overspannende kunstmatige lipide dubbellagen en positionering van membraaneiwitten boven porieopeningen bereikt.

Hoofdstuk 1 geeft een korte introductie over en motivatie voor gestuurd moleculair transport in bioanalyses met behulp van macromoleculaire nanotechnologie. Ook de context en inhoud van dit proefschrift wordt gepresenteerd in hoofdstuk 1.

In Hoofdstuk 2 wordt een literatuuroverzicht gegeven van verscheidene intelligente polymeerborstels in nanoporiën die gebruikt worden voor gestuurd transport. Hierin wordt ook de theorie, synthese en karakterisering van polymeerborstels beschreven. Daarbij worden in dit hoofdstuk ook voorbeelden gegeven van toepassingen van oppervlakte functionalisatie en gestuurd transport door poriën met stimulus responsive polymeerborstels.

Een aanvullend literatuuroverzicht over porie-overspannende lipide dubbellagen voor functionele analyses voor membraaneiwitten, aansluitend op het onderzoekswerk gepresenteerd in dit proefschrift, wordt in Hoofdstuk 3 gegeven. De rol van membraaneiwitten als medicijndoel wordt geïntroduceerd en ook de huidige beperkingen in de ontwikkeling van de functionele analyses worden uiteengezet. De vorming en karakterisering van kunstmatige lipide dubbellagen en de integratie van membraaneiwitten in de biomembranen maken ook deel uit van hoofdstuk 3. Aan bod komen ook specifieke voorbeelden, waarin het belang van ondersteuning door polymeren van deze kunstmatige lipide dubbellagen wordt beschreven.

Als onderdeel van het ontwerp van de modificatie van nanoporeuze platformen met polymeerborstels, zijn PMAA borstels vanaf het oppervlak van vlakke silicium oppervlakken gesynthetiseerd met surface-initiated atom transfer radical polymerization (SI-ATRP), dit is beschreven in Hoofdstuk 4. Groeikinetiek van de $\mathrm{pH}$-responsive 
polymeerlagen is onderzocht in mengsels van water en methanol met verschillende mengverhoudingen. Het door het $\mathrm{pH}$-verschil veroorzaakte zwellen en krimpen van de borstel is gekarakteriseerd met in situ ellipsometry. Een snelle en reversibele respons van de PMAA lagen, met zwelfactoren die toenamen bij de afname van de methanol hoeveelheid in het ATRP mengsel, werd waargenomen. Fourier transform infrared (FTIR) spectra, opgenomen tijdens titratie-experimenten, duidden op een verhoging van de effectieve $\mathrm{p} K_{\mathrm{a}}$ van de PMAA lagen naar $6.5 \pm 0.2$ ten opzichte van de bulk $\mathrm{p} K_{\mathrm{a}}$ van PMAA. Deze verschuiving is veroorzaakt door insluiting van ionen in de compacte borstel en brengt de verandering in fysieke toestand van de PMAA borstels dichter bij fysiologische omstandigheden. Dit is belangrijk voor biologisch georiënteerde toepassingen in geminiaturiseerde instrumenten met snelle reactietijden.

In Hoofdstuk $\mathbf{5}$ wordt de functionalisatie en karakterisering van nanoporeuze oppervlakken met putjes of kanalen met PMAA borstels beschreven. De borstels zijn gesynthetiseerd met SI-ATRP in een mengsel van water en methanol (1:1 volumeverhouding) om de bevochtiging van de poriën te verbeteren. De PMAA borstelgroei en het $\mathrm{pH}$-sensitive gedrag op de nanoporeuze oppervlakken zijn bevestigd door scanning electron microscopy (SEM), atomic force microscopy (AFM) en FTIR spectroscopie. AFM beeldvorming is ook gebruikt om de zwelling van de PMAA borstel, specifiek in de putjes gesynthetiseerd, waar te nemen door de $\mathrm{pH}$ van de vloeistof in contact met de polymeerborstels te wisselen tussen $\mathrm{pH} 4$ en $\mathrm{pH}$ 8. AFM beelden toonden openstaande nanoputten bij $\mathrm{pH} 4$ en gesloten putjes bij $\mathrm{pH}$ 8. Oppervlakken met nanokanalen zijn gefunctionaliseerd met PMAA borstels en onderzocht met cyclovoltammetrie bij verschillende $\mathrm{pH}$-waardes. De gefunctionaliseerde poriën hebben een hogere elektrische weerstand wanneer de borstel in een neutrale (open) toestand waren en een lagere elektrische weerstand wanneer de borstels geladen (gesloten) waren. De resultaten van deze metingen, gecombineerd met diffusie-experimenten met behulp van een fluorescent molecuul om mechanisch transport door de poriën aan te tonen, laten zien dat gestuurd transport van ionen en kleine moleculen door de PMAA borstel gefunctionaliseerde poriën mogelijk is. Al de resultaten gepresenteerd in hoofdstuk 5 rationaliseren het gebruik van nanoporeuze oppervlakken gefunctionaliseerd met PMAA borstels voor gestuurde doorgangtoepassingen, bijvoorbeeld in elektrochemische (bio)sensoren of kleppen op de nanoschaal.

In Hoofdstuk 6 wordt de functionalisatie met PMAA borstels van op silicium gebaseerde nanoporie grid chips beschreven, welke zijn gebruikt als platformen voor 
porie-overspannende lipide dubbellagen. De vorming van de kunstmatige lipide dubbellagen boven de gefunctionaliseerde poriën is bereikt door spontane liposoomscheuring en -fusie. Verhoogde membraanweerstand, bepaald met electrochemical impedance spectroscopy (EIS), en vrije diffusie van fluorescent gemerkte lipiden waargenomen met fluorescence recovery after photobleaching (FRAP) bevestigen de aanwezigheid van een lipide dubbellaag. Naast het ondersteunen van de lipide dubbelllaag, is nitrilotriacetic acid (NTA) gekoppeld aan de PMAA ketens voor de integratie van His-tagged membraaneiwitten. De plaatsing van membraaneiwitten langs de porieranden en de integratie in de porie-overspannende lipide dubbellagen is aangetoond met fluorescence microscopy. Het is ook aangetoond dat de eiwitdichtheid gevarieerd kan worden in een groot bereik zonder beïnvloeding van de vorming van de lipide dubbellaag. Hoewel verbeteringen nodig zijn voor elektrochemische metingen van membraaneiwitactiviteit, is al wel de potentie van het geïntegreerde platform voor analyses van ion channel membrane proteins gedemonstreerd.

Een toekomstvisie bestaande uit een discussie van de huidige beperkingen om analyse-instrumenten voor membraaneiwitten te ontwikkelen is terug te vinden in Hoofdstuk 7. Toekomstige ontwikkelingen voortvloeiend uit het werk gepresenteerd in dit proefschrift zijn: aan- en uitschakelen van individuele poriën voor gestuurd transport door van tevoren bepaalde poriën; analyse van transport van ionen en potentiele medicijnen met een laag molecuulgewicht per individuele nanoporie; en testen van de universele toepasbaarheid van het ontwikkelde analyseplatform door integratie van verschillende membraaneiwitten. 


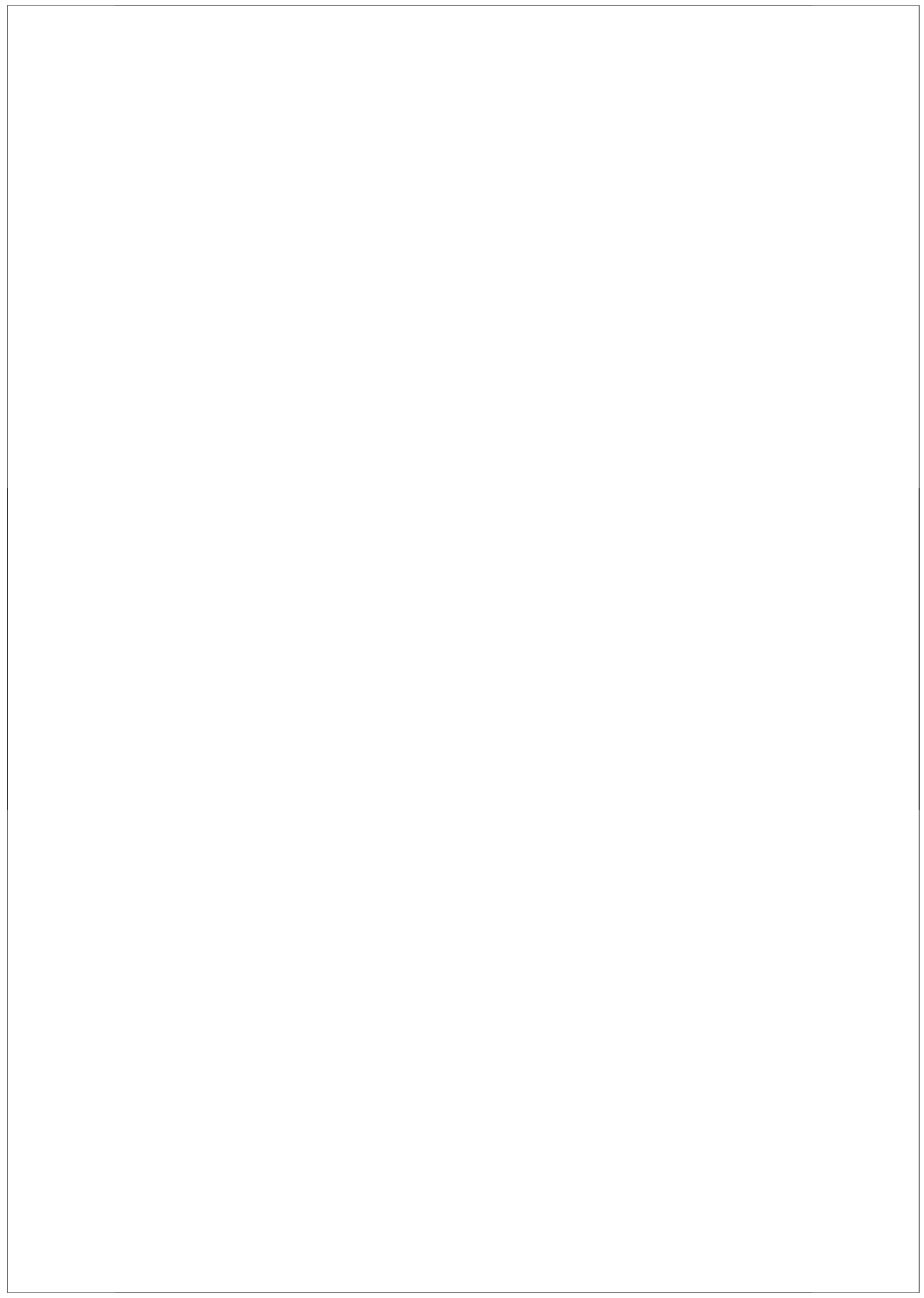




\section{Dankwoord}

Na bijna zes jaar in de MTP groep en vijf jaar als promovenda in deze groep is het schrijven van dit dankwoord het laatste onderdeel van mijn proefschrift dat nog geschreven moet worden. Aan de ene kant klinkt zes jaar lang, maar aan de andere kant is de tijd voorbij gevlogen. In de afgelopen jaren heb ik veel geleerd, veel beleefd en veel plezier gehad en hiervoor wil ik graag een aantal personen hartelijk bedanken. Maar aangezien ik niet iedereen bij naam zal noemen, wil ik ook iedereen die ik niet noem, maar dit wel leest, van harte bedanken voor je belangstelling in mij en mijn onderzoek.

Ten eerste wil ik graag mijn promotor, Julius Vancso, bedanken. Beste Julius, hartelijk bedankt dat ik onder jouw supervisie een promotieonderzoek mocht doen. Jouw MTP groep heb ik op meerdere vlakken ervaren als een zeer uitdagende werkomgeving en ik ben je dankbaar voor alles wat ik hierdoor geleerd heb. Ook ben ik heel blij dat ik het 'Promovendi voor de klas' programma kon volgen en daardoor bevestigd heb gekregen dat ik graag mijn carrière wil voortzetten in het onderwijs.

Dear Gabriella, we started together in MTP on the ASMENA project, you as a post-doc and I as a PhD student. Now when I am writing this, I am still a PhD student, but you developed from a post-doc to my daily supervisor and finally to my assistant promotor. I thank you for all your supervision, your ability to motivate me to try once more, looking for the positive points and your great help in writing. I enjoyed sharing an office, sharing a room during ASMENA meetings and your research stays in Enschede. Thank you very much for everything and all the best for your academic career and personal life.

Ook wil ik de twee stafleden Mark en Peter bedanken. Mark, bedankt voor het meedenken met en beantwoorden van al mijn scheikundige vraagstukken binnen en buiten het lab. Peter, na een presentatie kwam je altijd met een vraag en interessante publicaties. Peter, bedankt.

Ook zijn er natuurlijk nog de ondersteunende medewerkers binnen MTP. Eerst een hele belangrijke schakel binnen MTP. Beste Clemens, als er apparatuur even niet werkte of er was iets anders te regelen, je liet meteen alles uit je handen vallen en repareerde of regelde het. Clemens, hartelijk bedankt voor alles. Ook zijn er twee 'office managers' op het MTP secretariaat. Geneviève, bedankt voor het regelen van administratieve rompslomp, het behandelen van mijn kleinere en grotere vragen, en je belangstelling voor mij. Maar vooral bedankt voor de geweldige omslag van dit proefschrift. Ik was 
veeleisend, maar het is helemaal geworden zo als ik wilde. Hanke, bedankt voor het regelen van allerlei zaken en je interesse in mij.

My PhD project was part of the European Union research program ASMENA. Therefore I would like to thank all the ASMENA members, which I met during inspiring project meetings in Hungary, Switzerland, The Netherlands, France, and Sweden, where we also had lots of fun. Especially I would like to thank Sophie and Kaori for my two productive and very pleasant research stays in Switzerland. Thank you very much and all the best for the future.

I also would like to thank all the past and present MTP group members with whom I worked and enjoyed all the formal and informal MTP happenings. Also all the other persons at the UT who helped me or were interested in my research.

Aysegul, Michel, Bram, Lionel, Bart, and Joost thank you for correcting my chapters. Joost en Bram ook bedankt voor op het laatste moment corrigeren van mijn Nederlandse samenvatting.

Now it is time to thank my paranymphs, Aysegul and Bram. Aysegul, thank you for accepting this task. I would also like to thank you for the few but good conversations we had about work and our lives. It was great to be at your wedding. Good luck with finishing your PhD project and wish you all the best for the future. Bram, ook jij bedankt voor het zijn van mijn paranimf. Maar het was vooral heel leuk om je als kantoorgenoot te hebben. Elkaar helpen met werk gerelateerde zaken, maar ook kletsen, lachen, en lekker klagen gebeurde veelvuldig in ons kantoor. Heel veel succes met de tweede helft van je promotieonderzoek en veel geluk in de toekomst.

Ook wil ik al mijn familieleden en vrienden bedanken voor alle getoonde interesse en steun.

Hans en Jantje, Menno en Mayke, en Rutger ook jullie bedankt voor al jullie belangstelling voor mijn onderzoek, maar vooral alle gezelligheid, die altijd bij jullie te vinden is.

Marco, mijn broer, bedankt voor je onvoorwaardelijke steun in alles.

Papa en mama, jullie zijn mijn basis en zijn er altijd voor mij. Naast dat jullie trots zijn op mij, mogen jullie ook zelf trots zijn, want dit heb ik mede door jullie bereikt. Ik dank jullie vanuit het diepst van mijn hart. 
Evert, zonder jou naast me had ik dit niet gekund, al zal je zodra je dit leest het tegendeel beweren. Bovenal je relativeringsvermogen heeft me veel geholpen. Maar ook je grenzeloze vertrouwen in mijn kunnen, je zorgzaamheid voor mij, je humor en je liefde voor mij. Ik hou van je en samen is een gezellig, leuk en mooi avontuur!

Wilma 


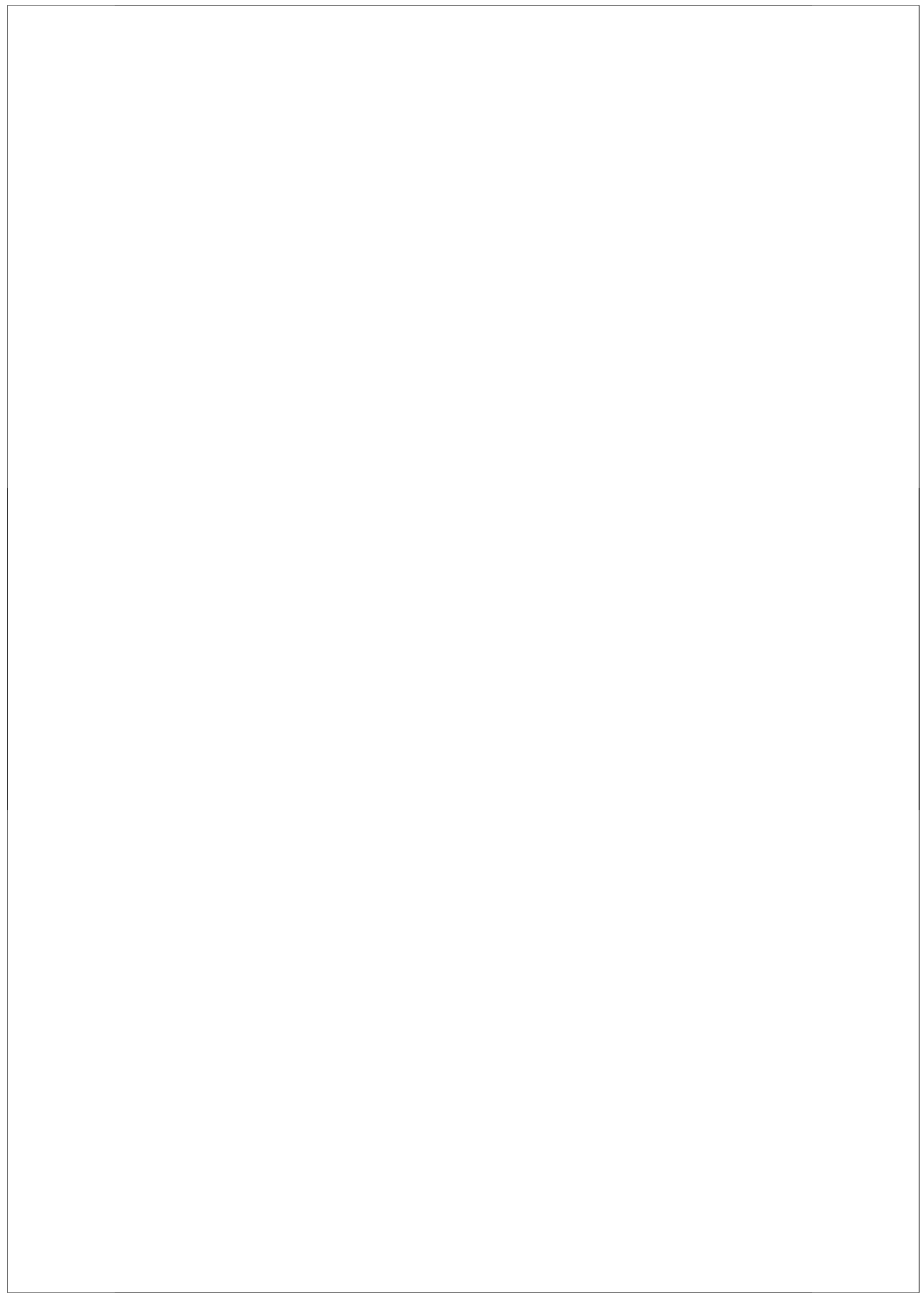




\section{About the author}

Gesina Wilhelmina (Wilma) de Groot was born on January $2^{\text {nd }}, 1983$, in Den Ham (Ov.), the Netherlands. She received her secondary school education (VWO) at CSG 't Noordik in Almelo and graduated in 2001. In the same year she started studying Chemical Engineering at the University of Twente in Enschede. She obtained her MSc. degree after performing her final MSc. research project in the Materials Science and Technology of Polymers (MTP) group of Professor G. Julius Vancso on 'Surface immobilization and patterning of polystyrene-block-poly(acrylic acid) vesicles on solid supports'. After graduation she continued in the same group as a PhD student. The results of her research are described in this dissertation.

Next to her PhD project, Wilma followed the $\beta$ XLinx-project 'Promovendi voor de klas', which facilitates PhD students in obtaining a teaching degree. Wilma expects to get her Master of Science Education and Communication in the spring of 2014. Subsequently, she aims to continue her career in the field of chemistry and science education at the secondary school level and/or the developments in this field. 


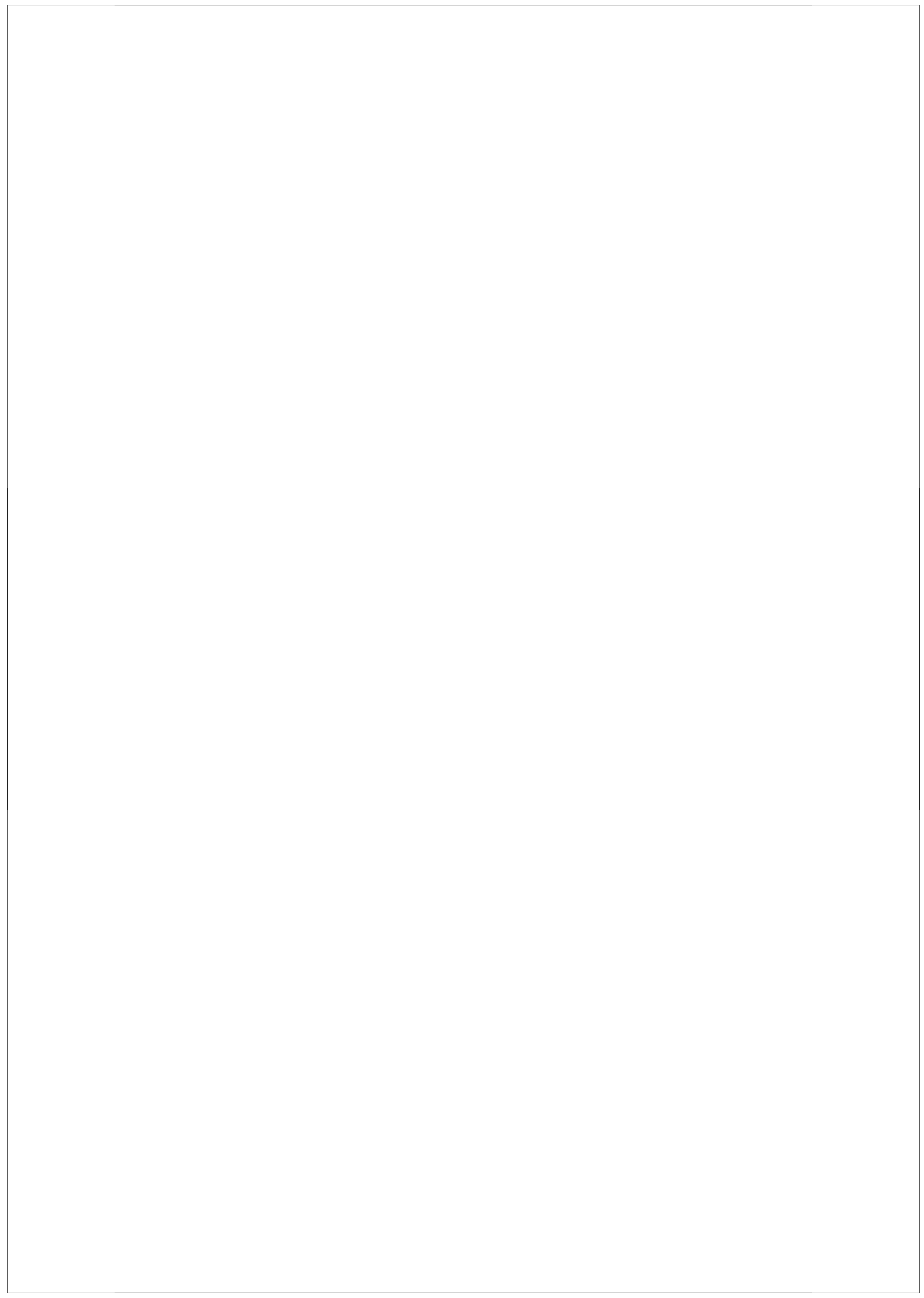

